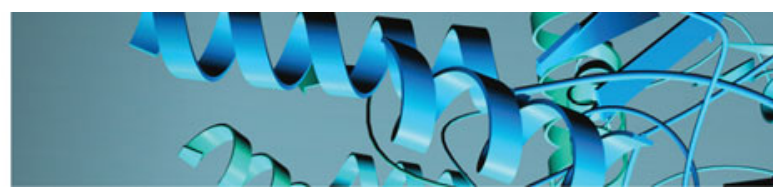

REVIEW

\title{
Protein dynamics and function from solution state NMR spectroscopy
}

\author{
Michael Kovermann ${ }^{\dagger \ddagger}$, Per Rogne ${ }^{\dagger}$ and Magnus Wolf-Watz* \\ Department of Chemistry, Umeå University, SE-901 87 Umeå, Sweden \\ Quarterly Reviews of Biophysics (2016), 49, e6, pages 1 of 43 doi:10.1017/S0033583516000019
}

Abstract. It is well-established that dynamics are central to protein function; their importance is implicitly acknowledged in the principles of the Monod, Wyman and Changeux model of binding cooperativity, which was originally proposed in 1965. Nowadays the concept of protein dynamics is formulated in terms of the energy landscape theory, which can be used to understand protein folding and conformational changes in proteins. Because protein dynamics are so important, a key to understanding protein function at the molecular level is to design experiments that allow their quantitative analysis. Nuclear magnetic resonance (NMR) spectroscopy is uniquely suited for this purpose because major advances in theory, hardware, and experimental methods have made it possible to characterize protein dynamics at an unprecedented level of detail. Unique features of NMR include the ability to quantify dynamics (i) under equilibrium conditions without external perturbations, (ii) using many probes simultaneously, and (iii) over large time intervals. Here we review NMR techniques for quantifying protein dynamics on fast (ps-ns), slow ( $\mu \mathrm{s}-\mathrm{ms})$, and very slow (s-min) time scales. These techniques are discussed with reference to some major discoveries in protein science that have been made possible by NMR spectroscopy.

\section{Introduction 2}

2. Development of NMR spectroscopy for quantification of protein dynamics 3

\section{Integration of NMR with other analytical methods 4}

4. Methods for quantifying dynamics by NMR spectroscopy 5

4.1. $\mathrm{R}_{1}$ and $\mathrm{R}_{2}$ relaxation and $\mathrm{hNOE} 6$

4.2. Relaxation dispersion 6

4.2.1. Exchange regimes 7

4.2.2. Determining the signs of chemical shifts from relaxation dispersion experiments 8

4.2.3. Exchange models 8

4.2.4. Data clustering to increase precision of fitted parameters 8

4.2.5. Properties/structures of minor states 8

4.2.6. Correlation between fitted and measured chemical shift differences 9

4.2.7. Structure determination of minor states from fitted chemical shifts 9

4.3. $\mathrm{R}_{1 \rho}$ rotating frame relaxation 9

4.4. ZZ-exchange 9

4.5. Chemical- and dark-state- exchange saturation transfer 10

4.6. Chemical shifts 10

4.7. Paramagnetic relaxation enhancement 11

4.8. Residual dipolar couplings 11

$\dagger$ These authors have contributed equally to this work.

\$ Present address: Department of Chemistry, University of Konstanz, Universitätsstrasse 10, 78457 Konstanz, Germany.

* Author for correspondence: Magnus Wolf-Watz, Department of Chemistry, Umeå University, SE-901 87 Umeå, Sweden. Email: magnus.wolf-watz@umu.se 
4.9. $\mathrm{H} / \mathrm{D}$-exchange 12

4.10. Real-time NMR 13

4.11. Ensemble approaches for analyzing protein dynamics 13

5. NMR-based breakthroughs in protein science (case studies) 13

5.1. Detection of functional high-energy protein states 14

5.1.1. Nitrogen regulatory protein C (NtrC) 14

5.1.2. DHFR 15

5.1.3. Ubiquitin 16

5.1.4. Adk 16

5.1.5. RNase A 17

5.1.6. Maltose binding protein (MBP) 17

5.1.7. Additional examples 18

5.2. Rate limitation in enzymatic catalysis 18

5.2.1. Adk 18

5.2.2. RNase A 19

5.2.3. DHFR 19

5.2.4. Tyrosine phosphatases 19

5.3. Intrinsic motions 19

5.4. Molecular recognition 20

5.4.1. Coupled folding/binding 20

5.4.1.1. Phosphorylated kinase inducible activation domain (pKID)-KIX 20

5.4.1.2. Virion protein $16(\mathrm{VP} 16)-\mathrm{hTAP}_{\mathrm{II}} 31 \quad 21$

5.4.1.3. SycE-YopE 21

5.4.1.4. $\mathrm{Z}_{\text {spa-1 }}$-staphylococcal protein A 21

5.4.1.5. Protein-small molecule interactions 22

5.4.1.6. Protein-DNA interactions 22

5.4.1.7. Protein-lipid interactions 22

5.4.2. Cracking or 'order-disorder-order' transitions 22

5.4.3. Coupled unfolding/binding 23

5.4.4. Folding by phosphorylation 23

5.4.5. Conformational entropy 23

5.4.5.1. Ligand binding 24

5.4.5.2. Negative binding cooperativity 24

5.4.6. Encounter complexes 24

5.4.6.1. The N-terminal domain of enzyme I (EIN) and phosphocarrier protein 25

5.4.6.2. Cytochrome c (Cc) and cytochrome c peroxidase (CcP) 25

5.5. Chaperonins 25

5.5.1. GroEL 25

5.5.1.1. Substrate binding 26

5.5.1.2. GroEl mediated protein folding 26

6. Outlook 27

\section{Acknowledgements 28}

\section{References 28}

\section{Introduction}

Nuclear magnetic resonance (NMR) spectroscopy is one of the most powerful tools available for structural biology. Rapid progress in the development of NMR hardware and software has made it into a mature technique, which is reflected in the fact that much modern NMR research focuses on its applications in the study of complex systems such as biological macromolecules. In such investigations, NMR is frequently used in an integrated fashion together with other analytical and computational techniques. This review focuses on the use of solution-state NMR spectroscopy to quantitatively characterize protein dynamics, and the insights into protein function that can be gleaned from such experiments. NMR is uniquely 
suitable for quantifying protein dynamics because (i) it can be used to quantify dynamics over a wide range of time scales, from picoseconds to hours; (ii) dynamics can be quantified under equilibrium conditions, with no need for external perturbations such as changes of temperature or pressure; (iii) dynamics can be quantified using many probes simultaneously, with atomic resolution; and (iv) a large set of orthogonal experimental approaches for quantifying dynamics have been developed. The fact that protein flexibility is an innate and crucial property of enzymes has been appreciated since the introduction of the induced fit theory (Bennett \& Steitz, 1978; Koshland, 1958), which builds on Emil Fischer's lock-and-key model (Fischer, 1894). Conformational changes in protein structures caused by substrate binding have historically been identified from $\mathrm{x}$-ray crystallography, and it has become evident that subtle structural changes can have profound effects on enzyme performance. For instance, sub-angstrom changes in the positions of amino acid residues in the active site of isocitrate dehydrogenase can change its kinetic parameters by several orders of magnitude (Mesecar et al. 1997). An expansion of the induced fit model is the conformational selection or population shift model, which builds on the Monod, Wyman and Changeux (MWC) model of cooperativity (Monod et al. 1965). This model predicts the existence of functional high-energy states of proteins that are sampled in the absence of a substrate or binding partner. NMR is uniquely suited for the detection and structural description of these high-energy states, as discussed in detail below. It is now textbook knowledge (Williamson, 2012) that proteins are dynamic and that structural states should be treated as ensembles that fluctuate around energy minima. This behavior is described by the energy landscape theory (Frauenfelder et al. 1991), which can be applied to protein dynamics using the principle of minimal frustration (Ferreiro et al. 2007, 2014; Jenik et al. 2012). NMR spectroscopy has developed in parallel with the energy landscape theory, and the two research fields are now converging to provide an unprecedented understanding of protein function.

Because NMR spectroscopy has been used to study the dynamics of many kinds of biological macromolecules, it would be impossible to present a comprehensive list of its use for this purpose in this review. We therefore do not discuss the use of NMR to study nucleic acid dynamics (Kimsey et al. 2015) and structure (Keane et al. 2015), or quantitative analysis of protein dynamics using solid state NMR spectroscopy (Lewandowski, 2013; Ullrich \& Glaubitz, 2013). We also do not cover the use of NMR to study protein folding (Dyson \& Wright, 2004) or intrinsically disordered proteins (Dyson \& Wright, 2005; Wright \& Dyson, 2015), although it is important to note that both fields are relevant to protein function. Furthermore, we do not attempt to provide a comprehensive list of important discoveries about protein function that have been made using solution state NMR experiments; our aim is to highlight the most important contributions from our perspective.

\section{Development of NMR spectroscopy for quantification of protein dynamics}

In the first part of this review we will discuss how modern high-resolution NMR spectroscopy has evolved into an exceptionally well-developed technique of great value in structural biology (Fig. 1). Based on the fundamental observations of Zeeman (1897) and Gerlach \& Stern (1922), Isaac Rabi discovered the magnetic properties of nuclei in 1936 and developed a resonance method for their detection. Bloch (1946) and Purcell et al. (1946) independently discovered the magnetic resonance of nuclei and the 'Bloch equations' were formulated. Later on, Gutowsky et al. (1953) and McConnell (1958) revised the Bloch equations to account for exchange effects between different sites in a molecule. Luz \& Meiboom (1963) published the first analytical solution for two-site exchange for any given set of experimental parameters valid in the fast exchange limit.

An important milestone in the development of multi-dimensional NMR spectroscopy was the introduction of twodimensional (2D) experiments by Jeener et al. (1979). This was followed by the development of the model-free approach for quantifying protein motion, which was presented by Lipari \& Szabo (1982a) and is important in the study of dynamics by NMR. The Lipari and Szabo theory applies in the Redfield limit of the Bloch-Wangsness-Redfield theory (Redfield, 1957, 1965; Wangsness \& Bloch, 1953). A subsequent fundamental contribution was made by Kay et al. (1989) with the development of inverse detected heteronuclear pulse sequences for determining the longitudinal and transversal relaxation rates $\left(R_{1}\right.$ and $R_{2}$, respectively) as well as the heteronuclear nuclear Overhauser effect ( $h \mathrm{NOE}$ ). Protein motions on the $\mu$ s-to-ms time scale have become widely experimentally accessible because of the relaxation-compensated Carr-Purcell-Meiboom-Gill (CPMG) pulse sequence introduced by Loria et al. (1999). It was later shown that the time required for these experiments can be significantly reduced by applying a constant time relaxation period (Skrynnikov et al. 2001).

Together, these contributions provide a robust theoretical and experimental basis for understanding the connections between protein dynamics and protein function (Boehr et al. 2006a; Eisenmesser et al. 2005; Tzeng \& Kalodimos, 2009). Among methods for studying protein dynamics, solution state NMR spectroscopy is unique in its ability to provide quantitative information on dynamics at atomic resolutions (i) under equilibrium conditions with no external perturbations based on the addition of co-solvents or changes in the experimental conditions (temperature, pressure, $\mathrm{pH}$ ), (ii) using multiple atomic probes in a single protein, and (iii) on time scales ranging over 18 orders of magnitude (Fig. 2) (Kempf \& Loria, 2003). While NMR research formerly suffered from size limitations that restricted its applicability in the analysis of larger systems, 


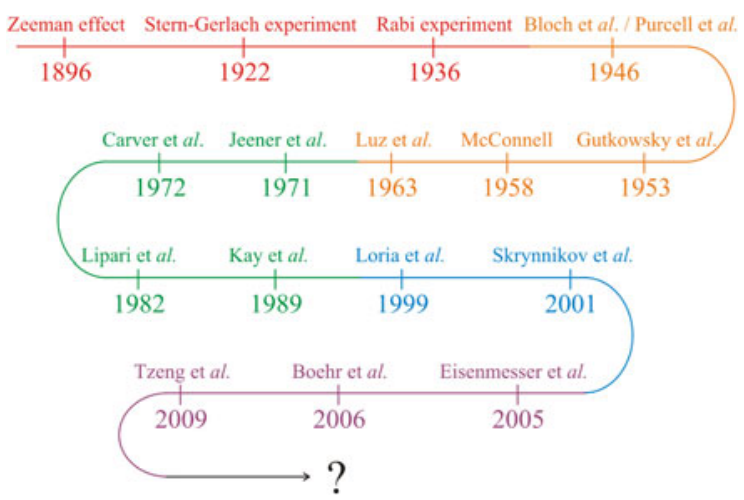

Fig. 1. Development of solution state NMR for quantification of protein dynamics. The curved arrow describes key developments and findings that constitutes the basis for quantification of protein dynamics from high-resolution solution state NMR spectroscopy. Basic discoveries are shown in red and are followed in order of prismatic colors by increasingly specific findings, which have led to the experimental setups used presently.

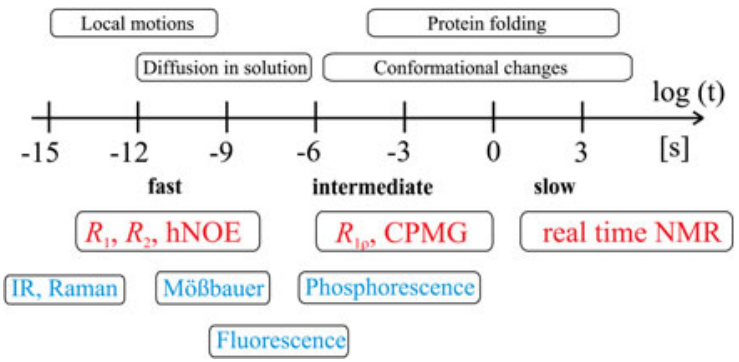

Fig. 2. Time scale of protein events and corresponding NMR techniques. The time scale available for detection of motional events in proteins by solution state NMR spectroscopy comprises more than 18 orders of magnitude as indicated with the logarithmic scale below the arrow. Dynamics can be divided into slow $\left(k_{\mathrm{ex}} \ll \Delta \omega\right)$, intermediate $\left(k_{\mathrm{ex}} \sim \Delta \omega\right)$, and fast $\left(k_{\mathrm{ex}} \gg \Delta \omega\right)$ events on the NMR time scale, where $k_{\mathrm{ex}}$ corresponds to the exchange rate constant of a dynamic process $\left(k_{\mathrm{ex}}=k_{\text {forward }}+k_{\text {reverse }}\right)$ and $\Delta \omega$ is the difference in chemical shifts for a two-site exchange model. Typical events occurring in proteins at these time scales are listed above the arrow. NMR experiments suitable for quantification of dynamics in a specific regime are colored in red and complementary techniques suitable for quantification of protein dynamics are colored in blue.

these have largely been abolished by the introduction of transverse relaxation optimized spectroscopy (TROSY)-based experiments (Pervushin et al. 1997; Tugarinov et al. 2003), new coherence transfer approaches (Fiaux et al. 2002; Riek et al. 2002) and the sensitivity improvements made possible by the widespread availability of cryogenic probes and high field magnets (Fiaux et al. 2002; Gelis et al. 2007; Mainz et al. 2013; Rudiger et al. 2002; Saio et al. 2014; Sprangers et al. 2005, 2007; Sprangers \& Kay, 2007; Tugarinov et al. 2004).

\section{Integration of NMR with other analytical methods}

A consequence of the maturity of NMR methods is that many NMR laboratories now focus their efforts on biological questions. Since most aspects of proteins' biological functions are complex, it is often necessary to investigate them using multiple orthogonal experimental techniques in order to obtain robust conclusions. Consequently, NMR is often one tool among many that are used in an integrated fashion in a given research project. This section of the review highlights some notable cases in which NMR was successfully combined with other experimental techniques to study protein dynamics. In addition many of the specific findings described in Section 5 were obtained through such integrated research efforts. NMR spin relaxation is a powerful tool for exploring protein dynamics, often providing important kinetic parameters such as microscopic rate constants or residence times in different states. However, to relate these kinetic parameters to structures, these NMR data must often be complemented with 3D structural information obtained using techniques such as $\mathrm{x}$-ray crystallography, separate NMR experiments, electron microscopy, small angle $\mathrm{x}$-ray scattering (SAXS), or wide angle $\mathrm{x}$-ray scattering (WAXS). Experiments using these methods are sometimes performed under different conditions to those used in solution-state NMR experiments; they may require the presence (or absence) of specific ligands or binding partners, or particular temperatures, $\mathrm{pH}$ values, or pressures. Nevertheless, the structures obtained using these methods are often used as models for the 
structural states observed by solution-state NMR. Many enzymes have been crystallized with and without ligands, resulting in the identification of multiple different conformations (Müller et al. 1996; Müller \& Schulz, 1992; Sawaya \& Kraut, 1997). In such cases, data from dynamic experiments can be interpreted in terms of interconversions between these states. Mutations of single amino acids that stabilize minor conformations predicted by dynamics can be used to draw conclusions regarding the conformation of these states (Fraser et al. 2009; Nikolova et al. 2011). Structures of stabilized minor states can potentially be determined by NMR or x-ray crystallography (Fraser et al. 2009), or their chemical shifts can be compared with relaxation dispersion data (Bouvignies et al. 2011; Grey et al. 2003; Korzhnev et al. 2010; Nikolova et al. 2011).

Computer based molecular dynamics simulations provide information that can be used in conjunction with dynamic data from NMR. In particular, simulations can provide the amplitudes of dynamic modes (Palmer, 2015), which generally cannot be determined from NMR spin relaxation experiments. The dependency is mutual since protein dynamics observed by NMR can be used to validate and develop the force fields used in molecular dynamics (MD) simulations. Limitations in computer performance and force field capabilities have previously restricted the use of MD simulations to interpret fast motions. However, it is now possible to perform simulations that extend into the ms regime (Lindorff-Larsen et al. 2011; Vanatta et al. 2015). The underlying spectral density function, which can be seen in simpler terms as the generalized order parameter based on the model-free formalism (Lipari \& Szabo, 1982a, b), can be calculated directly from MD simulations (Levy et al. 1981; Stafford et al. 2013). The order parameters obtained from MD simulations can then be compared with experimentally obtained spin relaxation parameters from $\operatorname{NMR}\left(R_{1}, R_{2}\right.$ and $\left.h \mathrm{NOE}\right)$, which are determined by analysis using the model-free formalism (Lipari \& Szabo, 1982a, b). This approach is exemplified by a recent study on cyclophilin A in which NMR experiments and simulations were used in tandem, suggesting that this enzyme catalyzes proline isomerization via an electrostatic handle mechanism (Camilloni et al. 2014).

SAXS experiments can be a valuable complement to NMR data for studying dynamics. For example, time-resolved SAXS (trSAXS) was combined with relaxation dispersion data by Phillips et al. (2014) in order to explore the relationship between internal motions and the activation of cellular inhibitor of apoptosis 1 (cIAP1). trSAXS was used to describe global conformational changes on a time scale of seconds while $R_{2}$-relaxation dispersion experiments were used to characterize dynamics on the millisecond time scale. The potential of combining SAXS with NMR (along with a range of other techniques) is also demonstrated by the work of the van Thor group on the photoactive yellow protein (Ramachandran et al. 2011).

SAXS and NMR can also provide complementary information on highly dynamic intrinsically disordered proteins (IDPs). NMR observables such as paramagnetic relaxation enhancements (PREs), residual dipolar couplings (RDCs) and NOEs can be combined with SAXS data to calculate an ensemble of structures representing the dynamic behavior of the IDP (Sibille \& Bernado, 2012). The same approach can also be used to study proteins with domains that are connected by flexible linkers.

Electron paramagnetic resonance (EPR) techniques like double electron-electron resonance spectroscopy (DEER) can be used to measure the distance between two spin labels attached to a protein (Hubbell et al. 2000). In one notable example, DEER experiments using spin-labeled frozen samples $(T=60 \mathrm{~K})$ of the photoactive yellow protein showed that the distance distribution of the labels changed on exposure of the photoreceptor to light (Ramachandran et al. 2011). In the same study, timeresolved pump-probe x-ray scattering (TR-SAXS/WAXS) (Cammarata et al. 2008) was used to demonstrate that the radius of gyration and particle size both increased when the protein was exposed to light (Ramachandran et al. 2011). Chemical shift perturbations and classical NOE data were combined with TR-SAXS/WAXS and DEER data to produce a model of the protein's dynamic light-exposed state (Ramachandran et al. 2011).

Complementary functional data are also essential when interpreting dynamic processes and attempting to determine their biological relevance. Kinetic rate measurements can be performed to correlate dynamic rate constants to overall activity levels. For example, a study on the protein dehydrofolate reductase showed that the rate of loop opening to release the substrate/ product was $11 \mathrm{~s}^{-1}$ (McElheny et al. 2005), which is about 20 times slower than the hydride transfer catalyzed by this enzyme as determined by a fluorescence based assay (Fierke et al. 1987). Similarly, the production of adenosine diphosphate (ADP) from adenosine monophosphate (AMP) and adenosine triphosphate (ATP) by Adenylate kinase (Adk) is monitored by a reduced nicotinamide adenine dinucleotide (NADH) absorption based coupled assay (Rhoads \& Lowenstein, 1968) and has been used to correlate overall catalytic rates with dynamic processes in the protein (Wolf-Watz et al. 2004). The examples listed in this section clearly show that combining NMR spectroscopy with other experimental techniques is a powerful way of addressing mechanistic questions in protein science.

\section{Methods for quantifying dynamics by NMR spectroscopy}

There exist many approaches for studying dynamics on different time scales using NMR spectroscopy. In this section we briefly summarize various methods that have proven to be useful for quantifying dynamics and relating them to protein function. 


\section{${ }_{4.1} R_{1}$ and $R_{2}$ relaxation and hNOE}

The longitudinal and transversal relaxation rates of proton-bound ${ }^{13} \mathrm{C}$ or ${ }^{15} \mathrm{~N}$ nuclei are sensitive to protein motions on the ps-to-ns time scale, as are the associated heteronuclear NOEs (Figs 2 and 3). The values of these parameters can be determined using established methods (Kay et al. 1989; Nirmala \& Wagner, 1988, 1989; Palmer et al. 1991), and the resulting relaxation data can be analysed using the model-free approach introduced by Lipari \& Szabo (1982a) and extended by Clore et al. (1990). Several key parameters can be quantified via their relationship with spectral density functions (Abragam, 1961), including the rigidity of the bond vector under investigation, which is expressed by the parameter $S^{2}$; the time scales of intramolecular motions, expressed by the correlation time $\tau_{\mathrm{e}}$; the overall level of molecular tumbling, expressed by the correlation time $\tau_{\mathrm{c}}$; and the contributions to chemical exchange, $R_{\mathrm{ex}}$. Notably, the order parameter $S^{2}$ is important in methods for quantifying entropic contributions to phenomena such as ligand binding to enzymes, as discussed in Section 5.4.5 (Diehl et al. 2009; Marlow et al. 2010; Seewald et al. 2000; Trbovic et al. 2009; Veglia \& Cembran, 2013); for a lengthier discussion, see the review by Wand (2013). Numerous relaxation studies have been conducted with the aim of relating inherent dynamic features on the ps-to-ns time scale to proteins' functional properties (Fenwick et al. 2014; Jankowski et al. 2012; Keramisanou et al. 2006; Kovermann et al. 2011; Lu et al. 2006; Miletti et al. 2011; Zeeb et al. 2003). This fast time scale spin relaxation methodology has also been successfully applied to larger protein systems such as Arginine Kinase (Davulcu et al. 2009). An elegant integrated approach for this purpose that combines room-temperature x-ray crystallography and NMR spectroscopy was presented by Wright and co-workers (Fenwick et al. 2014). Two important reviews of this field have been published (Sapienza \& Lee, 2010; Wand, 2001), which together provide a comprehensive overview of methods for studying fast time scale dynamics in proteins.

\subsection{Relaxation dispersion}

Many biological processes such as protein folding and conformational changes occur on the slow $\mu \mathrm{s}$-to-ms time scale and as such proteins dynamics in this motional window are of general interest for understanding protein function (Figs 2 and 3 ). Dynamics on the $\mu$ s-to-ms time scale can be characterized in terms of an exchange contribution, $R_{\mathrm{ex}}$, to the observed transverse relaxation rate, $R_{2}^{\text {obs }}$, using the following expression:

$$
R_{2}^{\mathrm{obs}}=R_{\mathrm{ex}}+R_{2}^{0}
$$

where $R_{2}^{0}$ is the intrinsic relaxation rate of the nuclei under investigation. For a two-site exchange $R_{\mathrm{ex}}$ depends on the population of the states $\left(p_{\mathrm{a}}, p_{\mathrm{b}}\right)$, the difference in chemical shifts $(\Delta \omega)$, the exchange rate constant $\left(k_{\mathrm{ex}}=k_{\mathrm{f}}+k_{\mathrm{r}}\right.$, where $k_{\mathrm{f}}$ and $k_{\mathrm{r}}$ are the forward and reverse rate constants, respectively) and the pulse repetition rate $\left(\tau_{\mathrm{cp}}\right)$. Under free precession conditions, the time-dependent evolution of the magnetization can be described by the McConnell equations (McConnell, 1958). Sequences of $180^{\circ}$ radiofrequency pulses executed with a frequency $v_{\mathrm{cp}}=1 / 4 \tau_{\mathrm{cp}}$ are known as CPMG pulse trains (Carr \& Purcell, 1954; Meiboom \& Gill, 1958) and enable detection of dynamic processes occurring on the $\mu$ s-to-ms time scale. Luz \& Meiboom (1963) presented a solution for a two-site exchange process assuming fast exchange $\left(\left(k_{\mathrm{ex}} / \Delta \omega\right) \rightarrow \infty\right)$ between site a and b:

$$
R_{2}=R_{20}+\frac{p_{\mathrm{a}} p_{\mathrm{b}} \Delta \omega^{2}}{k_{\mathrm{ex}}}\left(1-\frac{4 v_{\mathrm{cp}}}{k_{\mathrm{ex}}} \tanh \left\{\frac{k_{\mathrm{ex}}}{4 v_{\mathrm{cp}}}\right\}\right)
$$

with:

$R_{20}^{\mathrm{a}}=R_{20}^{\mathrm{b}}=R_{20}$.

A general solution for a two-site exchanging system was introduced by Carver \& Richards (1972) and further refined by Davis et al. (1994):

$$
R_{2, \mathrm{eff}}\left(\tau_{\mathrm{cp}}\right)=0.5\left(R_{2 \mathrm{a}}+R_{2 \mathrm{~b}}+k_{\mathrm{ex}}-\frac{1}{\tau_{\mathrm{cp}}} \cosh ^{-1}\left\{D_{+} \cosh \eta_{+}-D_{-} \cos \eta_{-}\right\}\right)
$$

with:

$$
\begin{aligned}
& D_{ \pm}=0.5\left(\frac{\psi+2 \Delta \omega^{2}}{\left.\sqrt{\psi^{2}+\phi^{2}} \pm 1\right)}\right. \\
& \eta_{ \pm}=\frac{\tau_{\mathrm{cp}}}{\sqrt{2}} \sqrt{\sqrt{\psi^{2}+\phi^{2}} \pm \psi} \\
& \psi=\left(R_{2 \mathrm{a}}-R_{2 \mathrm{~b}}-p_{\mathrm{a}} k_{\mathrm{ex}}+p_{\mathrm{b}} k_{\mathrm{ex}}\right)^{2}-\Delta \omega^{2}+4 p_{\mathrm{a}} p_{\mathrm{b}} k_{\mathrm{ex}}^{2}
\end{aligned}
$$




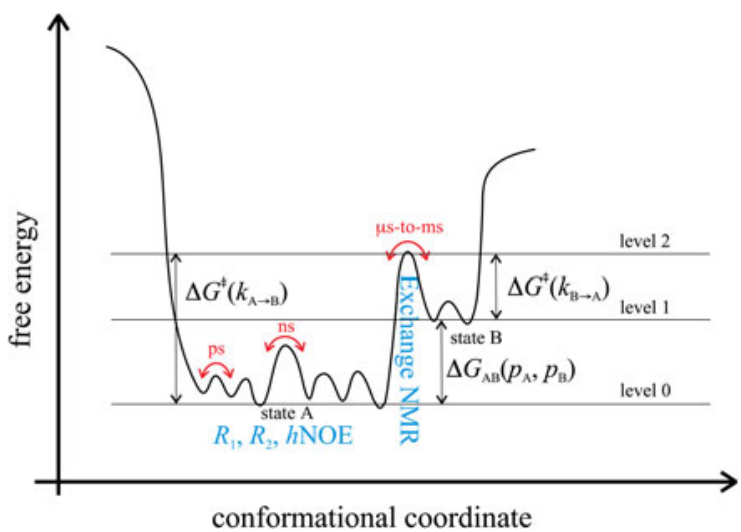

Fig. 3. Energy landscape visualizing dynamic motions on different time scales. State A (energy level 0) corresponds to the most stable ground state of a protein that can be studied with for instance $\mathrm{x}$-ray crystallography. State B (energy level 1) corresponds to a high-energy state that may be of functional importance and that can be studied with for instance, relaxation dispersion experiments. High-energy protein states are discussed extensively in Section 5. The transition state separating states A and B is indicated with energy level 2. NMR experiments that are suitable to observe ns-to-ps motions within one conformational state as well as $\mu$ s-to-ms motions for the transition between conformational states are depicted in blue.

$\phi=2 \Delta \omega\left(\mathrm{R}_{2 \mathrm{a}}-\mathrm{R}_{2 \mathrm{~b}}-\mathrm{p}_{\mathrm{a}} \mathrm{k}_{\mathrm{ex}}+\mathrm{p}_{\mathrm{b}} \mathrm{k}_{\mathrm{ex}}\right)$

$\Delta \omega=2 \pi \Delta v$.

It should be noted that even though Eq. (3) is a general solution for a two-site exchanging system, a linear correction must be applied to obtain an exact analytical solution (Baldwin, 2014).

A robust experimental technique in which a relaxation-compensated CPMG pulse sequence is used to monitor chemical exchange processes with time constants of between 0.5 and $5 \mathrm{~ms}$ was introduced by Loria et al. (1999). Time limitations for data acquisition were overcome by implementing a constant relaxation delay (Skrynnikov et al. 2001). In parallel, Tollinger et al. (2001) developed an approach that is applicable in the slow exchange limit, $k_{\mathrm{ex}} / \Delta \omega \rightarrow 0$ :

$$
R_{2 \mathrm{a}}^{\mathrm{eff}}=R_{2 \mathrm{a}}^{0}=k_{\mathrm{a}}-k_{\mathrm{a}} \frac{\sin \left(\Delta \omega /\left\{4 v_{\mathrm{cp}}\right\}\right)}{\Delta \omega /\left\{4 v_{\mathrm{cp}}\right\}}
$$

with:

$\left|R_{2 \mathrm{a}}^{0}-R_{2 \mathrm{~b}}^{0}\right| \ll k_{\mathrm{a}}, k_{\mathrm{b}} \ll 4 v_{\mathrm{cp}}$ and $v_{\mathrm{cp}}$ as the inverse of $\tau_{\mathrm{cp}}$.

The theoretical and experimental framework outlined above has been used extensively to detect motions on the $\mu \mathrm{s}$-to-ms time scale within proteins and understand their function (Beach et al. 2005; Bhabha et al. 2011; Boehr et al. 2006b; Eisenmesser et al. 2005; Farber \& Mittermaier, 2011; Farber et al. 2012; Korzhnev et al. 2010, 2004; Kovermann \& Balbach, 2013; Mulder et al. 2001a; Saio et al. 2014; Sugase et al. 2007; Tzeng \& Kalodimos, 2009, 2013; Whittier et al. 2013; Zeeb \& Balbach, 2005). Some notable examples of its use are discussed in detail in Section 5.

\subsubsection{Exchange regimes}

The window in which exchange parameters can be accurately quantified from relaxation dispersion data is defined by exchange rate constants $\left(k_{\mathrm{ex}}\right)$ of around $100-2000 \mathrm{~s}^{-1}$. However, by combining ${ }^{15} \mathrm{~N}$ and ${ }^{1} \mathrm{H}^{\mathrm{N}} \mathrm{CPMG}$ profiles with major state-induced ${ }^{15} \mathrm{~N}$ chemical shift changes (Vallurupalli et al. 2011), it is possible to increase the upper limit of this window to $4000-6000 \mathrm{~s}^{-1}$ (Sanchez-Medina et al. 2014). It is essential to establish the exchange regime for the process under investigation, which can be done quantitatively by analyzing the static field dependency of the exchange contribution $\left(R_{\mathrm{ex}}\right)$ to the observed transverse relaxation rate, the so-called $\alpha$-value (Millet et al. 2000). The $\alpha$-value is defined according to; $\alpha=\mathrm{d} \ln \left(\mathrm{R}_{\mathrm{ex}}\right) /$ $\operatorname{dln}(\Delta \omega)$. An estimation of $\alpha\left(\alpha^{\prime}\right)$ can be calculated using:

$$
\alpha^{\prime}=\frac{\left(B_{02}+B_{01}\right)}{\left(B_{02}-B_{01}\right)} \frac{\left(R_{\mathrm{ex} 2}-R_{\mathrm{ex} 1}\right)}{\left(R_{\mathrm{ex} 2}+R_{\mathrm{ex} 1}\right)}
$$

where $B_{0}$ is the static magnetic field and $R_{\mathrm{ex}}$ is the exchange contribution under different static fields with no applied CPMG field (i.e. $v_{\mathrm{CP}}$ equals zero). The subscript ' $n$ ' denotes the different static magnetic fields $(n=1,2)$. It has been shown that for slow exchange $0 \leqslant \alpha \leqslant 1$, for intermediate exchange $\alpha=1$, and for fast exchange $1 \leqslant \alpha \leqslant 2$. Since the extraction of relevant 
parameters is most effective under an intermediate exchange regime, it can be useful to manipulate the time scale of exchange such that the exchange process occurs within this window. The most convenient way to accomplish this is by varying the temperature (modulation of $k_{\mathrm{ex}}$ ) or the static magnetic field (modulation of $\Delta \omega$ ). In order to retrieve parameters with highprecision when fitting relaxation data to the Carver-Richards equation, it is useful to repeat a relaxation dispersion experiment at two different static magnetic field strengths. This approach significantly reduces any co-variation of fitted parameters that could otherwise preclude accurate quantification of protein dynamics (Kovrigin et al. 2006).

\subsubsection{Determining the signs of chemical shifts from relaxation dispersion experiments}

An inherent problem with chemical shift differences between major and minor states determined by fitting from relaxation dispersion experiments is that they are obtained as absolute values. This precludes their use as constraints when calculating the structures of minor states or as quantitative measures of protein activation. This problem was resolved when it was discovered that comparisons of chemical shifts in the indirect dimensions in either a pair of heteronuclear single quantum coherence spectroscopy (HSQC) spectra acquired at different magnetic fields or HSQC and heteronuclear multiple quantum coherence spectroscopy (HMQC) spectra acquired at a single field provide the sign of the chemical shift difference (Skrynnikov et al. 2002). This finding significantly expands the usefulness of chemical shifts determined by fitting from relaxation dispersion experiments.

\subsubsection{Exchange models}

As noted above, relaxation dispersion experiments enable the extraction of kinetic (rate constants), thermodynamic (populations), and structural (chemical shifts) parameters for a system undergoing exchange on the $\mu$ s-to-ms time scale. These parameters are quantified by fitting analytical or numerical solutions of the Bloch-McConnell equations to the observed data. The most commonly used exchange model is the two-state model, which has been demonstrated (typically by means of statistical

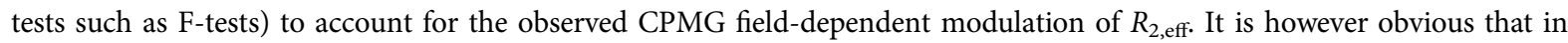
most cases a two-state model will represent a significant simplification of a system undergoing exchange. On the other hand the two-state assumption is very important and its use has enabled many major discoveries. Expansions to three-state models require data of outstanding quality (Sugase et al. 2007) and complexity, such as results from experiments conducted at multiple static magnetic field strengths or temperatures. In very favorable cases a three state model can be used without additional constraints such as addition of ligands or change of temperature (Meinhold \& Wright, 2011). There exist a few cases in which three-state models have successfully been applied to proteins undergoing exchange, such as the study by Grey et al. (2003) on the multiple states induced by disulfide bond isomerization in the basic pancreatic trypsin inhibitor, and the calculation of structural ensembles of folding intermediates for two Fyn SH3 domain variants reported by Korzhnev et al. (2004). It should be noted that the three-state model in Korzhnev et al. (2004), was applied under the assumption that (i) the chemical shift differences between exchanging states are temperature-independent, and (ii) the temperature dependence of the rate constants obeys standard transition-state theory (Fersht, 2000). Analyses based on a three-state model have also shown that a C-terminal extension in the PBX homeodomain with an unfolded main ground state also populates two states in which it is either folded or misfolded (Farber et al. 2012). The three-state model was applied by incorporating additional information (in this case, ligand-induced chemical shift changes) in the fitting procedure, and the paper includes a description of a gridbased search procedure that can be used to extract dynamic parameters based on a three-state model.

\subsubsection{Data clustering to increase precision of fitted parameters}

Slow protein fluctuations such as conformational changes involve concerted movements of large groups of atoms. Hence when such processes are studied with relaxation dispersion methodology it can be expected that individual probes (nuclei) should share $k_{\mathrm{ex}}$ values and populations of major and minor states. Global or cluster fits (assuming shared $k_{\mathrm{ex}}$ values and populations) of groups of nuclei are therefore justified on physical grounds. Global fits have the advantage of increasing the precision with which exchange parameters are estimated (Demers \& Mittermaier, 2009) but care must be taken with respect to how grouping is performed. Grouping can be performed by simply grouping nuclei, which yields exchange parameters similar to those obtained when nuclei are treated individually. Alternatively a structure-based approach can be used in which grouping is performed on the basis of secondary or tertiary structure elements and their arrangement. Regardless of the chosen approach, it is important to apply statistical tests such as those outlined by Ishima \& Torchia (2005) to avoid over-fitting and/or incorrect grouping.

\subsubsection{Properties/structures of minor states}

As noted above, relaxation dispersion experiments can be performed to identify the exchange of major protein ground states and high-energy minor states. Accurate evaluation of the properties and functional relevance of these minor states is essential 
for understanding the influence of dynamics on protein function. When characterizing minor states it is generally informative to compare fitted chemical shifts to observations obtained by independent measurements. The following sections describe both direct and indirect methods for defining the properties of minor states.

\subsubsection{Correlation between fitted and measured chemical shift differences}

In an extensive study on the thermodynamics and kinetics of ligand binding to the Fyn SH3 domain, fitted and calculated chemical shifts were used to confirm that the binding event can be accurately described with a two-state model. Hansen et al. (2008) showed convincingly that the chemical shifts obtained from relaxation dispersion experiments were robust by comparing chemical shifts determined directly from NMR spectra to shifts determined from fits to dispersion data. In ${ }^{15} \mathrm{~N}$ relaxation dispersion experiments using a sample with a ligand:protein ratio of around $1 \cdot 5$, the fitted chemical shifts obtained by regression of the CPMG data show an one-to-one agreement with chemical shifts obtained by direct measurement in NMR spectra (Demers \& Mittermaier, 2009). This correlation of the chemical shifts unambiguously demonstrated that the minor state corresponded to a ligand-bound protein structure. This example, which features tight experimental control over the major and minor states, demonstrates the validity of using fitted chemical shifts to identify the properties of minor states observed in CPMG experiments. Correlations between fitted chemical shift differences and experimentally determined chemical shifts for adjacent complexes in the catalytic cycle were also exploited in a study where the free energy landscape of dihydrofolate reductase was defined on the basis of CPMG experiments (Boehr et al. 2006b).

\subsubsection{Structure determination of minor states from fitted chemical shifts}

A more involved way of obtaining detailed structural information on minor states is to use chemical shifts obtained from CPMG experiments for structure calculations. This approach has been used to compute the structures of folding intermediates that are populated in two Fyn SH3 domain variants (Korzhnev et al. 2010, 2004) and to determine a minor state structure of a Lysozyme variant (Bouvignies et al. 2011). In the latter case, the structure calculation was performed by using chemical shifts for several nuclei from CPMG experiments to guide Rosetta loop building and refinement (Wang et al. 2007).

\section{$4.3 R_{1 \rho}$ rotating frame relaxation}

$R_{1 \rho}$ relaxation experiments measure NMR spin relaxation in the rotating frame (Palmer \& Massi, 2006) and can be used to monitor transverse relaxation rate constants on a $\mu$ s-to-ms time scale. They are closely related to the CPMG experiments described in Section 4.2 (Ishima \& Torchia, 1999), but $R_{1 \rho}$ experiments enable the observation of protein dynamics on a time scale that is around one order of magnitude faster time than is possible with CPMG-based experiments because they permit the use of higher refocusing frequencies and do not exceed the duty cycle of the CPMG pulse train. $R_{1 \rho}$ experiments use either near-resonant (Szyperski et al. 1993) or off-resonant (Akke \& Palmer, 1996; Zinn-Justin et al. 1997) radiofrequency pulses to lock the magnetization along the direction of the effective field, $\omega_{\mathrm{e}}$, in the rotating frame. The relaxation rate constant along the direction of the effective field, $R_{1 \rho}$, depends on (i) the amplitude of the applied radiofrequency field, $\omega_{\mathrm{a}}$; (ii) the frequency offset from the applied spin lock field, $\sum$; and (iii) the amplitude of the effective field, $\omega_{\mathrm{e}}=\left(\sum^{2}+\omega_{\mathrm{a}}^{2}\right)^{0.5}$.

The $R_{1 \rho}$ technique has been used to examine the dynamic interplay between mesophilic and thermophilic ribonuclease $\mathrm{H}$ variants (Butterwick \& Palmer, 2006), to probe the conformational exchange of an oxidative folding intermediate (Gross et al. 2010) or understand the function of a potassium channel ligand (Sher et al. 2014). In analogy to relaxation dispersion measurements, both the magnitude and the sign of the chemical shift difference $\Delta \omega$ of the exchanging species can be obtained from $R_{1 \rho}$ experiments (Trott \& Palmer, 2002).

\subsection{ZZ-exchange}

Farrow et al. (1994a) introduced a pulse-sequence for the simultaneous determination of ${ }^{15} \mathrm{~N}$ longitudinal relaxation and chemical exchange on time scales of ms to s (Farrow et al. 1994a). The so-called ZZ-exchange experiment is similar to an approach developed previously (Montelione \& Wagner, 1989) and has been used to investigate the slow folding and unfolding processes of an SH3 domain. The basic methodology (Farrow et al. 1994a; Montelione \& Wagner, 1989) was further refined by the incorporation of TROSY (Li \& Palmer, 2009), which was made possible by the adoption of a strategy for excluding differential line-broadening effects (Kloiber et al. 2011) and by using a composite intensity ratio with quadratic time dependence for data analysis (Miloushev et al. 2008):

$$
I(t)=\frac{a_{\alpha \beta}(t) a_{\beta \alpha}(t)}{a_{\alpha \alpha}(t) a_{\beta \beta}(t)-a_{\alpha \beta}(t) a_{\beta \alpha}(t)} \cong \gamma t^{2}
$$


where $a_{\alpha \beta}(t)$ is the peak amplitude of state $\beta$ detected on state $\alpha$ using a mixing period $t$ and $\gamma=k_{1} k_{-1}$ in which $k_{1} k_{-1}$ are the forward $\left(k_{1}\right)$ and pseudo-first-order reverse $\left(k_{-1}\right)$ rate constants for the two-site exchange between $\alpha$ and $\beta$.

The usefulness of the ZZ-exchange methodology has been established by its application to a wide range of macromolecules such as dihydrofolate reductase (DHFR) (Morgan et al. 1999), ubiquitin conjugated enzymes Mms2-Ubc13 (Markin et al. 2010), an SH3 domain (Demers \& Mittermaier, 2009), RNA (Latham et al. 2009), a protein kinase (Stewart \& Igumenova, 2012), chemokine (Tyler et al. 2012), and a multidrug transporter (Morrison et al. 2012).

\subsection{Chemical- and dark-state- exchange saturation transfer}

Chemical Exchange Saturation Transfer (CEST) (Bouvignies \& Kay, 2012a, b; Vallurupalli et al. 2012; Vallurupalli \& Kay, 2013) and Dark-state Exchange Saturation Transfer (DEST) (Fawzi et al. 2011, 2010, 2012, 2014; Libich et al. 2013) both rely on saturation transfer (Forsen \& Hoffman, 1963) to provide information on invisible states in chemical exchange with visible states. In a saturation transfer experiment it must be possible to saturate the invisible state without saturating the visible state (Anthis \& Clore, 2015). CEST and DEST experiments differ in the way they satisfy this prerequisite: CEST exploits a difference in resonance frequency between the two states whereas DEST exploits differences in line width. Both methods apply a saturation field with an offset from the visible state frequency if the invisible state is saturated, and the saturation will be transferred by chemical exchange to the visible state, leading to a decrease in the intensity of the latter. Plotting the intensity of the visible state against the offset of the saturation field will produce a saturation profile that can be analyzed using the McConnell equations (McConnell, 1958), giving the populations of the states and the kinetic parameters for the exchange. In addition, the invisible state's chemical shift (in CEST) or the $R_{2}$ relaxation rate (in DEST) will be obtained.

CEST can detect minor states that are in slow exchange $\left(k_{\text {ex }}\right.$ between 10 and $\left.500 \mathrm{~s}^{-1}\right)$ provided that they are populated to at least $1 \%$. Faster exchange $\left(k_{\mathrm{ex}}>500 \mathrm{~s}^{-1}\right)$ approaches the intermediate exchange regime in which the two states do not have different chemical shifts. The sensitivity for the lower state is dependent on the applied saturation field. However, the chemical shift difference between the two states limits the field strength between 20 and $30 \mathrm{~Hz}$ (Vallurupalli et al. 2012), which limits the size of populations and speed of exchange that can be measured in this way. No such limitations on the maximum usable field strength apply in DEST experiments (in which the saturation field strength is $>100 \mathrm{~s}^{-1}$ ), allowing smaller populations to be detected. DEST is effective at approximately the same time scale as CEST $\left(k_{\mathrm{ex}}\right.$ between 10 and $\left.100 \mathrm{~s}^{-1}\right)$, but in certain favorable cases its range can be extended to both slower $\left(k_{\mathrm{ex}}<10 \mathrm{~s}^{-1}\right)$ and faster time scales $\left(k_{\mathrm{ex}}>1000 \mathrm{~s}^{-1}\right)$ (Anthis \& Clore, 2015).

\subsection{Chemical shifts}

The chemical shift is the most readily obtained NMR parameter and is always quantified for various nuclei in NMR spectroscopy. Since the chemical shift is extremely sensitive to the local magnetic environment it is also sensitive to the conformational state of a biomolecule. In the event of fast exchange between the two structural states (a and b), the observed chemical shift $\left(\omega_{\text {OBS }}\right)$ is given by the following simple but very useful equation:

$$
\omega_{\mathrm{OBS}}=p_{\mathrm{a}} \omega_{\mathrm{a}}+p_{\mathrm{b}} \omega_{\mathrm{b}}
$$

where $p_{\mathrm{a}}$ and $p_{\mathrm{b}}$ are the statistical weights (or populations) of states a and $\mathrm{b}$, and $\omega_{\mathrm{a}}$ and $\omega_{\mathrm{b}}$ are the corresponding chemical shifts. If the chemical shifts of the two exchanging states can be measured in independent experiments, it is possible to compute the free energy difference between these two states for any condition where the states are in fast exchange according to:

$$
\Delta G^{0}=-R T \ln \left(p_{\mathrm{a}} / p_{\mathrm{b}}\right)
$$

where $R$ is the gas constant and $T$ the absolute temperature.

The analysis of chemical shifts in this way clearly reveals the equilibrium distribution between the exchanging structural states. The biggest challenge in analyzing chemical shifts is to quantify the chemical shifts of the 'pure' a and b states. For enzymes, one option is to approximate the chemical shifts of states $\mathrm{a}$ and $\mathrm{b}$ from apo and inhibitor-bound states, respectively. While this approach is useful, it is important to recall that both of these reference states can also be involved in various exchange processes that may complicate the analysis. Nevertheless, analyses of chemical shifts have provided significant insights into several systems and chemical shifts are often used as a measure of a reaction coordinate between the active and inactive states of proteins (Ådén et al. 2012; Ådén \& Wolf-Watz, 2007; Li et al. 2008; Masterson et al. 2010, 2011b; Olsson \& Wolf-Watz, 2010; Volkman et al. 2001). An alternative approach for quantification of chemical shifts is the projection analysis (Selvaratnam et al. 2012). In this approach, the chemical shift difference between states a and $b$ is described as a vector $(\mathrm{AB})$ and the chemical shift of a state under investigation is projected onto the $\mathrm{AB}$ vector. The resulting angle $(\cos \theta)$ and activation vectors (defined by the magnitude of the projection onto $\mathrm{AB}$ ) are useful tools in judging the global nature of an activation process, as illustrated for the cAMP-dependent guanine nucleotide exchange factor EPAC (Selvaratnam et al. 2012). 


\subsection{Paramagnetic relaxation enhancement}

The paramagnetic relaxation enhancement (PRE) effect was firstly described by Solomon (1955). It arises from magnetic dipolar interactions between a nucleus and the unpaired electrons of a spin label, which lead to an increased rate of relaxation. The large magnetic moment of the unpaired electron in the paramagnetic metal ion or nitroxide label enables the PRE to be detected at large distances - up to $35 \AA$ (Clore \& Iwahara, 2009; Gillespie \& Shortle, 1997a, b). PRE experiments can thus be used to define long-range restraints for computing the structures of proteins bearing appropriate spin labels. Experiments of this sort have been used to study the structures of metal-binding proteins that already contain a paramagnetic ion or can readily be induced to bind to one (Bertini et al. 2001). In addition, they can be used with proteins that have been labeled with probes based on nitroxides (Kosen, 1989) or metals (Gaponenko et al. 2000). PRE rate constants are measured as the difference between the relaxation rates of paramagnetic and diamagnetic samples. The diamagnetic sample is normally obtained by either reducing the nitroxide tag or exchanging the bound paramagnetic metal ion with a diamagnetic one. A summary of pulse sequences for PRE rate constants measurements of different nuclei was presented by Clore \& Iwahara (2009).

PREs can be used to obtain information about dynamic processes on a fast time scale. The exchange rate constant, $k_{\mathrm{ex}}$, must be much bigger than the difference between the relaxation rates of the two states; in practical terms, this means that $k_{\text {ex }}$ must be on the order of $1000 \mathrm{~s}^{-1}$ (Anthis \& Clore, 2015). The magnitude of the experimentally determined PRE varies with the inverse sixth power of the distance, $r$, between the paramagnetic center and the studied nucleus:

$$
\Gamma_{2}^{\mathrm{HN}}=\frac{1}{r^{6}}\left\{\frac{1}{15}\left(\frac{\mu_{0}}{4 \pi}\right)^{2} \gamma_{\mathrm{H}}^{2} g^{2} \mu_{\mathrm{B}}^{2} S(S+1)\left(4 \tau_{c} \frac{3 \tau_{c}}{1+\omega_{\mathrm{H}}^{2} \tau_{c}^{2}}\right)\right\}
$$

where $r$ is the distance between the paramagnetic center and the observed nucleus, $\mu_{0}$ is the permeability of vacuum, $\gamma_{\mathrm{H}}$ is the gyromagnetic ratio, $g$ is the electron g-factor ('Landé-factor'), $\mu_{\mathrm{B}}$ is the electron Bohr magneton, $S$ is the electron spin quantum number, $\tau_{\mathrm{c}}$ is the PRE correlation time, and $\omega_{\mathrm{H}} /(2 \pi)$ is the nuclear Larmor frequency (Bloembergen \& Morgan, 1961; Solomon, 1955).

Due to the $r^{-6}$ dependency, a three-fold decrease in distance will yield almost a thousand-fold increase in the PRE. Moreover, since the apparent PRE $\left(\Gamma_{2 \text {,app }}\right)$ is the population weighted average between the PREs for two states a and b, even a $1 \%$ population of the minor state can have a large effect on the $\Gamma_{\mathrm{app}}$ if $k_{\mathrm{ex}} \gg\left(\Gamma_{2, \mathrm{~A}}-\Gamma_{2, \mathrm{~B}}\right)$ (Iwahara \& Clore, 2006). In such cases, minor states can be identified on the basis of a mismatch between measured PREs and PREs calculated on the basis of a structure of the major state a. An ensemble approach can then be used to fit the PRE data more accurately, revealing the presence of the minor state or states (Tang et al. 2006).

\subsection{Residual dipolar couplings}

In liquid state NMR spectroscopy, dipolar couplings (DC) (see Eq. (10)) average to zero due to the isotropic tumbling of molecules, and this effect simplifies recorded NMR spectra. However, since DC carry structural information on both local and global scales (Esteban-Martin et al. 2014) it can be useful to reintroduce a small amount of the dipolar coupling (on the order of 1\%) (Bax, 2003). This can be accomplished by partial alignment of molecules through the use of anisotropic media such as liquid crystalline media (Tjandra \& Bax, 1997), stretched polyacrylamide gels (Sass et al. 2000; Tycko et al. 2000), or pf1 phages (Hansen et al. 1998). The resulting DC is called residual dipolar coupling (RDC) (Gayathri et al. 1982; Tolman et al. 1995), or RDC, and can be accurately quantified for covalently bonded nuclei such as the backbone ${ }^{1} \mathrm{H}_{-}{ }^{15} \mathrm{~N}$ correlation (Ottiger et al. 1998; Tjandra et al. 1996). Today RDCs are routinely used in liquid NMR-based structure determination protocols and can increase the precision of structures significantly (Clore \& Gronenborn, 1998; Clore \& Schwieters, 2006; Tjandra et al. 1997).

$$
\mathrm{DC}=D_{\mathrm{a}}\left(3 \cos ^{2}-1+\frac{3}{2} R \sin ^{2} \theta \cos 2 \varphi\right)
$$

The $D C$ between two nuclei connected by an internuclear vector with an orientation, $\Omega$, in the eigenframe of the alignment tensor, $D$, is dependent on the polar angles $(\theta, \varphi)$. $D$ has eigenvalues $D_{\mathrm{xx}}, D_{\mathrm{yy}}$ and $D_{\mathrm{zz}}$ (Clore et al. 1998). The axial component of $D$ corresponds to $D_{\mathrm{a}}=D_{\mathrm{zz}} / 2$ and the rhombicity of $D$ is given by $R=2 / 3\left(\left(D_{\mathrm{xx}}-D_{\mathrm{yy}}\right) / D_{\mathrm{zz}}\right)$.

There are multiple ways of using RDCs to study the dynamics of proteins. They are well-suited for comparing the structural state of a protein in solution to structures obtained from x-ray crystallography (Bernado \& Blackledge, 2004; Bouvignies et al. 2005, 2006; Clore \& Schwieters, 2004b; Salvatella et al. 2008), as shown elegantly for various states of the HIV-1 protease (Roche et al. 2015) and arginine kinase (Niu et al. 2011). As an extension of this approach, it is also possible to determine the distribution of structural states in proteins by using ensemble methods (Esteban-Martin et al. 2010, 2014; Fenwick et al. 
2010; Maciejewski et al. 2011; Vammi et al. 2014) in which an ensemble of conformers is generated and RDCs computed from this ensemble are compared with experimental values. This technique depends on the calculation of alignment tensors using tools such as prediction of alignment from structure (PALES) (Zweckstetter \& Bax, 2000) or ALMOND programs (Almond \& Axelsen, 2002), grouping of conformers into clusters, and careful analysis to avoid over-fitting of data. Direct refinement of structures against experimental $R D C$ s has also been performed to compute a structural ensemble of ubiquitin that reflects motions occurring on time scales up to the ms range (Lange et al. 2008). RDCs can also be used as experimental input in MD simulations (Schwieters \& Clore, 2001). Under favorable conditions, the RDCs of such minor states can be quantified on the basis of relaxation dispersion experiments performed in anisotropic media (Vallurupalli et al. 2007); these measurements can then provide structural details of minor states. Another way of retrieving dynamical information from $R D C s$ is by using them to compute order parameters (Lakomek et al. 2008a; Peti et al. 2002). RDCs are sensitive to motions that are either faster or slower than the protein's rotational correlation time $\left(\tau_{\mathrm{m}}\right)$, so order parameters derived from RDCs are sensitive to motions occurring over time scales ranging from ps to ms. This was demonstrated in a study on ubiquitin in which $R D C$-derived order parameters (Meiler et al. 2001) were consistently found to be smaller than the corresponding Lipari-Szabo order parameters (Lakomek et al. 2005). RDCs have provided particularly important results in studies on IDPs. (Bernado et al. 2005; Jensen et al. 2013; Ulmer et al. 2005).

\subsection{H/D-exchange}

The basic principles of H/D exchange experiments were presented by Linderstrøm-Lang in the 1950s and are summarized in Eqs. (11) and (12) and (Hvidt \& Nielsen, 1966). Suppose that a state that is unavailable for exchange, for example due to hydrogen bonding $\left(\mathrm{NH}_{\text {closed }}\right)$, is in equilibrium with a state that is capable of hydrogen exchange $\left(\mathrm{NH}_{\text {open }}\right)$. The rate constant of the hydrogen exchange step, $k_{\mathrm{rc}}$, can be approximated as the intrinsic exchange rate constant for a random coil (Bai et al. 1993), which depends on the amino acid sequence, temperature, and $\mathrm{pH}$ value (Bai et al. 1993). The observed exchange rate constant, $k_{\mathrm{obs}}$, depends on three different rate constants as described in Eq. (12) (Wildes \& Marqusee, 2004). Depending on the ratio between $k_{\mathrm{cl}}$ and $k_{\mathrm{rc}}$ two different limiting cases can occur: EX1 where $k_{\mathrm{rc}} \gg k_{\mathrm{cl}}$, and EX2 where $k_{\mathrm{rc}} \ll k_{\mathrm{cl}}$. During EX1 all opening events will lead to an exchange, simplifying Eq. (12) to: $k_{\mathrm{obs}}=k_{\mathrm{op}}$, consequently this rate constant corresponds directly to the rate of the opening event. Under EX2, which is more commonly observed in practice than EX1, only a fraction of all opening events will lead to exchange. In this case Eq. (12) is reduced to $k_{\mathrm{obs}}=\left(k_{\mathrm{op}} / k_{\mathrm{cl}}\right) k_{\mathrm{rc}}$ or $k_{\mathrm{obs}}=K_{\mathrm{op}} k_{\mathrm{rc}}$, where $K_{\mathrm{op}}=\left(k_{\mathrm{op}} / k_{\mathrm{cl}}\right)$ is the equilibrium constant for the process that makes the proton available for exchange; this could be the disruption of one or more hydrogen bonds (Wildes \& Marqusee, 2004). Based on this equilibrium, local stabilities can be calculated using the equation of Englander \& Kallenbach (1983). The nature of the events leading to the exchange can also be studied, distinguishing between full unfolding and local fluctuations (Skinner et al. 2012a). Rate constants of exchange can be correlated to the solvent exposure of specific hydrogens, although these correlations only hold for hydrogens on the surface that are not involved in any hydrogen bonding within the protein; surface hydrogens of the latter sort exchange like hydrogens in the center of the protein (Skinner et al. 2012b).

$\mathrm{H} / \mathrm{D}$-exchange experiments measure slow dynamics occurring over time scales of seconds or longer. Measurements of H/D exchange on such time scales are generally performed by dissolving the protein in a fully deuterated solvent and then monitoring the disappearance of amide proton signals over time by acquiring a series of spectra, typically ${ }^{1} \mathrm{H}-{ }^{15} \mathrm{~N}-\mathrm{HSQCs}$. Faster methods of acquiring 'HSQC-like' spectra such as SOFAST-NMR (Schanda \& Brutscher, 2005; Schanda et al. 2005), Ultra SOFAST (Gal et al. 2007), and Band-Selective Short Transient (BEST) (Rennella et al. 2014) have been suggested as ways of increasing the achievable time resolution and enabling the study of faster exchange processes that occur over tens of seconds rather than minutes. Faster exchange processes can also be studied by using techniques where magnetization is transferred by exchange between water and amide protons, like MEXICO (Gemmecker et al. 1993) and refined variants of these techniques (Koide et al. 1995). Other experiments that use magnetization transfer to study hydrogen exchange between proteins and solvents have been developed to reduce artifacts introduced by alternative magnetization transfer mechanisms. Examples of such experiments are phase-modulated clean chemical exchange (CLEANEX-PM) (Hwang et al. 1998) and solvent exchange spectroscopy (SOLEXY) (Chevelkov et al. 2010). These techniques have made it possible to study hydrogen exchange processes on the millisecond time scale. In the limit of slow exchange it is even possible to obtain hydrogen exchange rate constants for minor states using an NMR experiment designed to detect chemical exchange saturation transfer $-{ }^{15} \mathrm{~N}$-based TROSY CEST (Long et al. 2014).

$$
\begin{gathered}
N H_{\text {closed }} \stackrel{k_{\mathrm{op}}}{\rightleftharpoons} N H_{\mathrm{open}} \stackrel{k_{\mathrm{rc}}}{\rightarrow} N D \\
k_{\mathrm{cl}} \\
k_{\mathrm{obs}}=\frac{k_{\mathrm{op}} k_{\mathrm{rc}}}{k_{\mathrm{cl}}+k_{\mathrm{rc}}}
\end{gathered}
$$




\subsection{Real-time NMR}

For very slow processes (i.e. processes occurring over time scales longer than seconds) it is possible to follow dynamics in real-time with NMR (Kumar \& Balbach, 2015). This approach requires a perturbation of the system in order to get an experimentally observable signal. The perturbation may be the addition of a binding partner or denaturant, or a change of temperature. Real-time NMR has been used in a number of seminal studies on cytochrome C (Roder et al. 1988), ribonuclease A (Udgaonkar \& Baldwin, 1990), and also other proteins (Balbach et al. 1995; Harper et al. 2004; Haupt et al. 2011; Hoeltzli \& Frieden, 1995; Udgaonkar \& Baldwin, 1990) and RNA (Cao et al. 2010; Wenter et al. 2005). A direct approach based on using NMR to assess slow dynamics of binding and folding was reported by Binolfi et al. (2012), that followed the binding and folding of a truncated version of thioredoxin (residues 1-93, TRX1-93) upon addition of a peptide containing the C-terminal region of the full protein (residues 94-108, TRX94-108). The rate constant of protein folding induced by the interaction between the two parts was measured in a real-time NMR experiment in which a series of ${ }^{1} \mathrm{H}^{15} \mathrm{~N}$ HET-SOFAST-HMQC spectra were acquired over the course of the folding reaction. This made it possible to follow the development of peaks corresponding to the folded thioredoxin. An overall folding rate constant of $0.016 \mathrm{~min}^{-1}$ was determined, corresponding to a folding half-life of $42 \mathrm{~min}$ (Binolfi et al. 2012). A temperature-based perturbation was applied in a study conducted by the Wolf-Watz group on the dissociation kinetics of the 'hetero-dimeric' Yersinia protein $\mathrm{Ysc}_{\mathrm{C}}$, in which a series of $1 \mathrm{D}{ }^{1} \mathrm{H}$ NMR experiments were acquired at a temperature of $37^{\circ} \mathrm{C}$ (Frost et al. 2012). It was shown that dissociation paralleled the secretion of effector proteins, which is a prerequisite for Yersinia infectivity. In addition, a real-time ${ }^{31} \mathrm{P} N M R$ approach was recently developed by Wolf-Watz and co-workers to quantify Adk activity (Rogne et al. 2015). The strength of this assay is that $k_{\text {cat }}$ can be determined in the absence of coupling enzymes and activity can therefore readily be studied in solutions containing co-solvents. This is important because the assay was developed to enable quantification of Adk activity in a switchable ionic liquid (Rogne et al. 2015).

\subsection{Ensemble approaches for analyzing protein dynamics}

One of the limitations of NMR spectroscopy is that it is inherently difficult to quantify the amplitude of motions, whether they occur on fast (ps-to-ns) or slow ( $\mu$ s-to-ms) time scales. One way of solving this problem is by studying structural ensembles (Clore \& Schwieters, 2004a, b; Iwahara et al. 2004) that are refined against experimental data that are sensitive to protein motions. Multiple implementations of this idea have been proposed but they all have several common steps: (1) generation of structural ensembles, (2) refinement against experimental data, (3) selection of a minimal structural ensemble that satisfactorily explains the experimental data, (4) cross-validation to reduce over-fitting, and (5) analysis of the structural ensemble in a functional or biophysical context.

Here we will briefly summarize the approach to these points described by Lindorff-Larsen et al. (2005), who developed a method called dynamic ensemble refinement (DER) and used it to obtain a structural ensemble of ubiquitin that is consistent with ${ }^{15} \mathrm{~N}$ order parameters $\left(S^{2}\right)$ reflective of motions on the ps-to-ns time scale. Ensemble refinement against both NOEs and order parameters was achieved using biased molecular dynamics (Paci \& Karplus, 1999) and a simulated annealing protocol starting from an x-ray structure of ubiquitin (Vijay-Kumar et al. 1987). Cross-validation of the ensemble was performed by comparing values computed from the ensemble to experimental RDCs and ${ }^{3} \mathrm{~J}$ couplings that were excluded from the ensemble refinement process. It was found that both the scalar couplings and RDCs predicted on the basis of the DER ensemble were more accurate than those predicted on the basis of $\mathrm{x}$-ray structures or an NMR ensemble derived from NOEs alone. Analysis of the DER ensemble provided new biophysical insights, suggesting that while the protein's core is tightly packed, it nevertheless exhibits appreciable fluid-like character. Another ubiquitin ensemble that was constructed by refinement against $R D C s$ and is sensitive to motions on a ps-to-ms time scale is described in some detail in Section 4 (Lange et al. 2008). Ensemble approaches have also been used to visualize transient encounter complexes (Bashir et al. 2010; Tang et al. 2006), quantify enzyme dynamics (Esteban-Martin et al. 2014), describe the solution structures of intrinsically disordered proteins (Jensen et al. 2013, 2014; Silvestre-Ryan et al. 2013), and probe correlated motions in proteins (Bouvignies et al. 2005; Bryn Fenwick et al. 2014; Clore \& Schwieters, 2004a; Fenwick et al. 2011).

\section{NMR-based breakthroughs in protein science (case studies)}

The ongoing development of solution state NMR methods has been accompanied by numerous significant contributions to the mechanistic understanding of protein function. Although not covered here, one of the biggest contributions has arguably been the development and application of NMR methods for determining the structures of biological macromolecules (Härd et al. 1990; Ikura et al. 1992; Keane et al. 2015; Nagata et al. 1999; Wuthrich, 2001; Zhou et al. 1998). It is becoming evident that major breakthroughs in understanding protein function will increasingly be made by multidisciplinary teams capable of 
applying several different analytical and computational techniques, and that NMR spectroscopy can be a central component of such efforts but will have to be integrated with other methods such as isothermal titration calorimetry (ITC), $\mathrm{x}$-ray crystallography, SAXS, single molecule fluorescence resonance energy transfer (smFRET) and functional assays (see Section 2). This section thus focuses on breakthrough findings that have been accomplished at least in part with spin relaxation experiments. We must stress that we have made no effort to be comprehensive, and that the works chosen for inclusion merely reflect our perspective on the field; there are certainly many other important discoveries that could have been included in the following sections.

\subsection{Detection of functional high-energy protein states}

It is becoming increasingly evident that the induced fit model is not sufficient to explain the complexity of macromolecular interactions. The energy landscape theory of protein structure and dynamics predicts that a protein samples different conformations in dynamic equilibrium (Ferreiro et al. 2014; Frauenfelder et al. 1991; Jenik et al. 2012; Miyashita et al. 2005). The relative weights of the different conformations follow statistical thermodynamic distributions, with the time scales of conformational exchange being determined by the heights of the corresponding energy barriers. In cases where the barrier heights are low relative to the Boltzmann energy $\left(k_{\mathrm{B}} T\right)$, thermally driven fluctuations can lead to an appreciable population of different conformations. It should be noted that the concept of conformational sampling was introduced as long ago as 1965 with the MWC model for cooperativity, which assumes that a substrate-free oligomeric enzyme can populate tense (T) and relaxed (R) states (Monod et al. 1965). In the conformational selection or population shift model, a ligand selectively binds to one of the conformations or sub-states that are sampled in solution (Boehr et al. 2009; Bosshard, 2001; Csermely et al. 2010; Foote \& Milstein, 1994; Leder et al. 1995; Lindley et al. 1993; Michielssens et al. 2015; Oh et al. 1993). Hence, the ligand does not induce a structural change but instead acts to redistribute the statistical weights of sub-states that are sampled even in the substrate's absence. The model can, in fact, be described as a one-site (i.e. $n=1$ ) MWC model. As is made clear in the following sections, NMR has provided convincing evidence that many proteins populate active conformations under substrate- or target-free conditions. Consequently the energy landscape (Frauenfelder et al. 1991) of some proteins innately permits the sampling of active-like conformations (Miloushev et al. 2008). However, this discovery does not mean that a specific ligand binding mechanism is best described with a conformational selection model. Flux analyses have shown that ligand and protein concentrations are central in determining whether conformational selection or induced fit pathways dominate. It is in fact possible for a conformational selection model to dominate at low substrate concentrations but to be replaced by induced-fit binding at higher substrate concentrations, as was demonstrated to be the case for DHFR (Hammes et al. 2009). A generic problem with the conformational selection model is that active states of enzymes may be unavailable to substrates due to steric occlusion (Sullivan \& Holyoak, 2008). Therefore, to firmly establish that conformational selection is the dominating pathway it is necessary to study the observed rate constant for binding over a wide range of concentrations for both interacting species (Gianni et al. 2014; Vogt \& Di Cera, 2012). However, experiments of this sort are far from trivial to perform for most binding reactions. Another possibility is to combine results from kinetic experiments obtained using a stopped-flow setup with lineshape analysis of NMR signals as described in a contribution from the Kern laboratory, which showed that differences in a cancer drug's binding affinity to Abl and Src kinases are dependent on the equilibrium constant of an induced-fit step (Agafonov et al. 2014). The sections below discuss selected NMR studies that have contributed significantly to unraveling the functional importance of high-energy protein states. We have also highlighted some of the technical aspects that have enabled the quantification of dynamics in different model systems.

\subsubsection{Nitrogen regulatory protein C (NtrC)}

Two component signaling systems are crucial for signal transduction in bacteria. As the name suggests, they have two evolutionarily conserved components: a histidine kinase and a response regulator, the latter of which has receiver and output domains (Parkinson \& Kofoid, 1992). These systems handle various signaling tasks relating to processes such as chemotaxis and metabolic adaption to changes in carbon and nitrogen availability; for a more extensive overview, please see the work of Parkinson \& Kofoid (1992). Phosphorylation of an aspartate residue in the receiver domain of NtrC (NtrC $\left.{ }^{r}\right)$ stimulates oligomerization of the dimeric output domain and thus down-stream activation. To understand the structural consequences of $\mathrm{NtrC}^{\mathrm{r}}$ phosphorylation, the Wemmer laboratory developed a protocol to study this inherently unstable species (Kern et al. 1999). NMR samples of phosphorylated $\mathrm{NtrC}^{\mathrm{r}}$ were prepared by adding a large excess of carbomylphosphate together with $\mathrm{Mg}^{2+}$. Under these conditions the samples remained stably phosphorylated for $36 \mathrm{~h}$. To enable acquisition of 3D datasets of sufficient quality, the authors co-added multiple data-sets (more effective stabilization was subsequently achieved by mimicking phosphorylation with $\mathrm{BeF}_{3}^{-}$(Gardino et al. 2009; Hastings et al. 2003)). The NMR structure of phosphorylated NtrC (Kern et al. 1999) exhibits significant structural changes relative to the un-phosphorylated state (Volkman et al. 1995). These changes are confined to one face (denoted the ' 3445 -face') of the molecule and extend over the region between $\alpha$-helix 3 


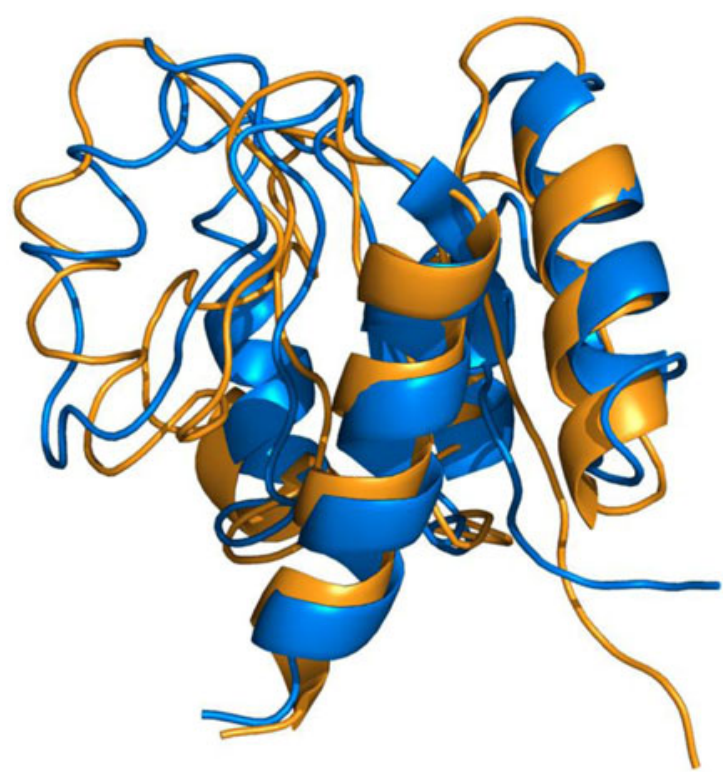

Fig. 4. Two state allosteric behavior of NtrC. Shown is a structural superimposition of phosphorylated NtrC (orange) and nonphosphorylated NtrC (blue) indicating the structural difference between active and inactive states, respectively. This figure was adapted from reference Kern et al. (1999).

and $\beta$-strand-5 (Fig. 4). An NMR structure of $\mathrm{BeF}_{3}^{-}-\mathrm{NtrCr}$ confirmed the overall features of the conformational change, although some differences with respect to the orientations of some segments were identified (Hastings et al. 2003). In a subsequent study, the authors designed experiments that ultimately showed that the structural change depends on the redistribution of already existing structural states (Volkman et al. 2001). That is, the un-phosphorylated species samples the active conformation, and in this state the key aspartate residue is primed for phosphorylation. This conclusion was primarily drawn on the basis of 'Model-free' driven deconvolution (Clore et al. 1990; Lipari \& Szabo, 1982a) of $R_{\mathrm{ex}}$ contributions to observed $R_{2}$ relaxation rates. The $R_{\mathrm{ex}}$ contributions of the phosphorylated species are quenched in comparison with both the wild type and a constitutively active mutant. These relaxation data were interpreted to mean that the phosphorylated species is 'locked' into the active conformation, leading to a quenching of $R_{\mathrm{ex}}$, but the $R_{\mathrm{ex}}$ contribution of the mutant and un-phosphorylated forms remains strong due to the sampling of both active and inactive conformations. This interpretation was buttressed by the observation of linear chemical shift perturbations for $\mathrm{NtrC}^{\mathrm{r}}$ variants with varying activity. Overall, this study elegantly describes MWC-like two-state allosteric behavior in a single domain protein. The findings related to NtrC were based on the assumption of a two-state model with exchange between inactive and active species. This assumption both explained the experimental data and enabled the development of a new concept for the activation of a single-domain protein. Two more recent studies on this protein have identified the molecular transition pathways between active and inactive NtrC states (Pontiggia et al. 2015; Vanatta et al. 2015).

\subsubsection{DHFR}

DHFR is an enzyme found in all organisms that catalyzes the reduction of 7, 8-dihydrofolate (DHF) to 5, 6, 7, 8-tetrahydrofolate (THF) with hydride transfer from the cofactor NADPH (Schnell et al. 2004). THF is essential for purine and thymidylate synthesis and thus for cell growth and proliferation. The reaction catalyzed by DHFR is dependent on five kinetic intermediates (Fierke et al. 1987) for which structural models derived from x-ray crystallography are available (Sawaya \& Kraut, 1997). These intermediates are known as E:NADPH, E:NADPH:DHF, E:NADP ${ }^{+}: T H F$, E:THF and E:NADPH:THF where 'E' stands for the enzyme DHFR. In NMR experiments, the Michaelis complex corresponding to the E:NADPH: DHF intermediate has been mimicked using the ternary complex E:NADP ${ }^{+}$:folate. In a spin relaxation study of the five kinetic and structural intermediates during DHFR catalysis, Wright and co-workers established a firm coupling between DHFR function and conformational dynamics (Boehr et al. 2006a; Schnell et al. 2004). Under substrate-saturating conditions, these five intermediates collectively represent a full catalytic cycle in which either the cofactor and/or products or substrates are bound to the enzyme. Notably, under the conditions imposed during the NMR experiments, all of the observed relaxation effects could be attributed to conformational changes in the enzyme. This was also shown explicitly by conducting relaxation dispersion experiments at different $\mathrm{NADP}^{+}$concentrations. Relaxation dispersion was acquired for ${ }^{15} \mathrm{~N}$ (Loria et al. 1999; 
Tollinger et al. 2001) and ${ }^{1} \mathrm{H}^{\mathrm{N}}$ (Ishima \& Torchia, 2003) nuclei at two static magnetic field strengths. The five different intermediates exhibited specific kinetic behavior signatures that were obtained from their relaxation dispersion patterns. The authors propose a model where each of the different ground states (i.e. the five intermediates described above) is in exchange with the ground state that precedes it and the one that follows it in the catalytic cycle. Consequently, the enzyme is primed for the subsequent step in the cycle by sampling the corresponding structural state. In addition it was found that several of the microscopic steps connecting the five intermediates are rate limited by conformational transitions. These conclusions were drawn by comparing fitted chemical shifts from relaxation dispersion experiments to direct measurements in NMR spectra, which showed (among other things) that there is a striking linear dependency of chemical shifts between the E:THF and E: NADP+:THF states for residues that are sensitive to cofactor binding (Boehr et al. 2006b).

\subsubsection{Ubiquitin}

Posttranslational modification of proteins by ubiquitin is central in many cellular pathways, although the best understood function of ubiquitination is in proteasome-mediated proteolysis (Harper \& Schulman, 2006). Ubiquitin binding domains bind non-covalently to ubiquitin and thereby control different cellular events (Hicke et al. 2005). Interestingly, the ubiquitin components of complexes of ubiquitin with different target proteins exhibit a remarkable level of structural heterogeneity (Lange et al. 2008). This suggests that ubiquitin's ability to bind to a very wide range of proteins is partly due to an appreciable degree of structural plasticity in its apo state. RDCs (Tjandra \& Bax, 1997; Tolman et al. 1995) are sensitive to motions that occur over ps to ms (Meiler et al. 2001, 2001, 1997; Peti et al. 2002) and thus fill a gap between the time windows that can be studied using Lipari-Szabo order parameters (Lipari \& Szabo, 1982a, b) (sub- $\tau_{\mathrm{c}}$ motion) and relaxation dispersion experiments (Loria et al. 1999; Tollinger et al. 2001) (between $50 \mu \mathrm{s}$ and $100 \mathrm{~ms}$ ). In an extensive RDC-based study, the laboratories of Griesinger and de Groot developed a method (denoted EROS) that enabled a precise determination of a structural ensemble of apo ubiquitin that is sensitive to motions in the ps- to ms- regime (Lakomek et al. 2005, 2008b); this regime is henceforth referred to as the supra- $\tau_{\mathrm{c}}$ regime. The ensemble refinement was performed by using $42 R D C$ datasets (Briggman \& Tolman, 2003; Ottiger \& Bax, 1998; Ruan \& Tolman, 2005). Notably, the EROS ensemble covers the complete range of ubiquitin conformations as observed in 46 different ubiquitin complexes (Lange et al. 2008). In a recent combined computational and NMR study, the dynamics of ubiquitin were explored in more detail, showing that the binding selectivity for ubiquitin targets can be tuned by perturbing ubiquitin's pre-existing conformational equilibrium (Michielssens et al. 2014). For most residues, the order parameters derived from the EROS ensemble are lower compared with those predicted by Lipari-Szabo analysis (Chang \& Tjandra, 2005). Analysis of the supra- $\tau_{\mathrm{c}}$ regime thus reveals additional mobility, in good agreement with the structural heterogeneity observed in the EROS ensemble. In summary it is evident that ubiquitin has innate dynamic properties that enable engagement with target proteins through conformational selection mechanisms, i.e. a multitude of different structural states are sampled in absence of target proteins and the presence of a specific target can select a specific ubiquitin structure. Finally, it is important to note that while this section focused on EROS, several other approaches for interpreting dynamics on the basis of RDCs exist (Bouvignies et al. 2006, 2007; Tolman, 2002; Tolman et al. 2001; Ulmer et al. 2003).

\subsubsection{Adk}

Adk catalyzes the magnesium-dependent reversible interconversion of AMP and ATP into two ADP molecules. Adk is vital for cellular energy-homeostasis and present in all organisms. The enzyme harbors two distinct nucleotide binding subdomains that are denoted ATPlid and AMPbd, respectively. Adk undergoes a large conformational change in response to ligand binding that shuttles the protein into a closed and active conformation where phosphoryl transfer occurs (Rhoads \& Lowenstein, 1968). Because of its favorable properties - specifically, its tendency to yield excellent NMR spectra (Ådén \& Wolf-Watz, 2007; Schrank et al. 2009), catalysis of a reversible reaction, and large conformational changes (Müller et al. 1996; Müller \& Schulz, 1992) - Adk has emerged as one of the principal model systems for analyzing the linkage between dynamics and enzymatic turnover (Arora \& Brooks, 2007; Hanson et al. 2007; Nagarajan et al. 2011; Schrank et al. 2009; Shapiro et al. 2009, 2000; Shapiro \& Meirovitch, 2006). In an extensive multi-approach effort, the Kern laboratory has shown that substrate-free Adk from the hyperthermophilic bacterium Aquifex aeolicus samples 'closed like' structures (Henzler-Wildman et al. 2007). This conclusion was drawn from (i) an x-ray structure containing three molecules in the asymmetric unit in which ATPlid and AMPbd adopted orientations that lay on a trajectory from open to closed structures, (ii) ${ }^{15} \mathrm{~N}$ relaxation dispersion measurements that detected motion on a time scale of $950 \pm 150 \mathrm{~s}^{-1}$ at $10^{\circ} \mathrm{C}$ in hinge regions and also in parts of both nucleotide binding sub-domains, (iii) paramagnetic relaxation enhancement experiments that showed enhanced relaxation in regions that are expected to be far apart in the open state but in close proximity in the closed state and (iv) single molecule FRET measurements that sampled opening/closing events with reaction kinetics of around $7000 \mathrm{~s}^{-1}$ at $20^{\circ} \mathrm{C}$. Taken together, these results suggest that apo Adk samples structural states that lie on a trajectory leading to the catalytically competent closed state. Hence the dynamics that are relevant for substrate binding are already 
present in the substrate-free enzyme and the enzyme exists in a conformational equilibrium before substrate addition (Henzler-Wildman et al. 2007). This pre-equilibrium has also been observed in ensemble analyses of RDCs measured for apo Adk (Esteban-Martin et al. 2014). The pre-existing equilibrium can be perturbed in the directions of closure or opening by addition of the osmolyte TMAO or by removal of one inter-domain hydrogen bond, respectively. Notably, these changes in the equilibrium constant are mirrored by compensatory effects on $k_{\text {cat }}$ and $K_{\mathrm{M}}$ such that the specificity constant $\left(k_{\text {cat }} / K_{\mathrm{M}}\right)$ remains invariant (Ådén et al. 2012; Kovermann et al. 2015). This effect, denoted ' $k_{\text {cat }}$ versus $K_{\mathrm{M}}$ ' compensation, stems from the fact that Adk is rate limited by conformational dynamics (see the section below), and is apparently evolutionarily robust against mutations that simultaneously affect $k_{\text {cat }}$ and $K_{\mathrm{M}}$ values. A more intuitive variation in $k_{\text {cat }} / K_{\mathrm{M}}$ values, i.e. a direct scaling of $k_{\text {cat }} / K_{\mathrm{M}}$ versus the population of a high-energy state, has been observed for the phosphorylation of phospholamban (Masterson et al. 2011b) and the Dbl homology domain of Vav1 (Li et al. 2008). The compensatory effects on $k_{\text {cat }}$ and $K_{\mathrm{M}}$ that have been observed in Adk are phenomenologically related to enthalpy-entropy compensation (Dunitz, 1995; Leffler, 1955), which is well-known to be important for protein folding (Fersht, 2000) but has also been observed in bio-molecular interactions (Cooper et al. 2001). The catalytic model for Adk catalysis (Wolf-Watz et al. 2004) contains an additional highenergy state corresponding to a substrate-bound open conformation. This state has now been directly observed by NMR spectroscopy (Kovermann et al. 2015) and it was shown that the interconversion of this high-energy state with a ground state structure that has been characterized by $\mathrm{x}$-ray crystallography is rate limiting for catalysis (as discussed further below).

\subsubsection{RNase A}

Quantification of enzyme dynamics that are relevant for substrate turnover is most readily performed for enzymes that catalyze reversible reactions (Eisenmesser et al. 2002; Wolf-Watz et al. 2004). However, many enzymes such as proteases catalyze irreversible reactions. For these enzymes, alternative approaches must be used to mimic and characterize Michaelis complexes (Michaelis \& Menten, 1913). One option that was explored in a study on RNase A (Beach et al. 2005) reported by the Loria laboratory was to use a small unreactive molecule that mimics the contacts that are expected in the Michaelis complex. RNase A primarily catalyzes the cleavage of single stranded RNA between purines and pyrimidines (Katoh et al. 1986). Determination of a x-ray structure (at $2 \cdot 0 \AA$ ) of a complex between RNase A and the dinucleotide $5^{\prime}$-phosphotymidine (3'-5') pyrophosphate adenosine 3'-phosphate (pTppAp) (Russo \& Shapiro, 1999) established that this small molecule is a relevant mimic of the Michaelis complex for RNase A catalysis. An additional important aspect of pTppAp is that its $K_{\mathrm{D}}$ for RNase A binding is sufficiently low - only $16 \mathrm{nM}$ (Kovrigin et al. 2003) - that any contributions from ligand association and dissociation can be safely neglected in the analysis of relaxation dispersion experiments. Analysis of relaxation dispersion experiments (Loria et al. 1999; Mulder et al. 2001b) showed that both apo and pTppAp bound RNase A contain a large set of residues with motions on the order of $1000 \mathrm{~s}^{-1}$ at $T=283 \mathrm{~K}$. Notably, these residues cluster in the same regions in the two forms of the enzyme and given the $K_{\mathrm{D}}$ value for pTppAP binding it was concluded that the dynamics observed in the presence of pTppAp are intra-molecular and independent of association and dissociation kinetics. Hence, the dynamics observed in the model Michaelis complex are already present in the apo enzyme. Based on a comparison of the exchanging states' chemical shift signs as determined using a HSQC/HMQC approach (Skrynnikov et al. 2002), and a direct measurement of the difference between the apo and pTppAp states, the authors concluded that the minor state observed in the apo state resembles the substrate-bound enzyme while the minor state of the pTppAp-bound complex resembles the conformation of the apo state. Subsequent studies on RNase A have shown that its dynamic processes are orchestrated through networks that are operational over long distances within the enzyme (Doucet et al. 2011; Kovrigin \& Loria, 2006; Watt et al. 2007). For instance the global dynamic process observed in pTppAp-bound RNaseA can be disturbed by introducing the substitution D121A: even though position 121 is distant from the active site and does not influence the active site properties, the D121A substitution causes a $90 \%$ reduction in $k_{\text {cat }}$. Consequently, the slow dynamics observed for the wild type enzyme in the Michaelis complex, which involve a concerted process occurring at $1900 \mathrm{~s}^{-1}$, are replaced by a sequence of two processes occurring at 2400 and $730 \mathrm{~s}^{-1}$, respectively (Kovrigin \& Loria, 2006). Hence concerted dynamic processes in enzymes are dependent on a fine balance of interactions and can be highly sensitive to subtle changes.

\subsubsection{Maltose binding protein (MBP)}

MBP is responsible for maltose uptake (Boos \& Shuman, 1998) and consists of two domains that are connected by three linker segments. Structures of apo (Sharff et al. 1992) and holo (Spurlino et al. 1991) states determined by x-ray crystallography have revealed a hinge-bending motion within the linker region resulting in a $35^{\circ}$ rigid body reorientation of the relative domain orientations (Sharff et al. 1992). MBP has been extensively characterized by NMR spectroscopy in both the apo and holo states, and RDC analysis showed that these two states predominantly populate the crystallographic structures in solution (Evenäs et al. 2001). PRE can be utilized to detect minor states (Iwahara \& Clore, 2006; Tang et al. 2006) that cannot be observed using methods based on RDCs, which are linear weighted averages of all species present in solution. (Tang et al. 
2007). Using PRE experiments, it was shown that the holo state in solution is well-described by the crystallographic holo structure. In contrast, the apo state was found to display PRE values that are inconsistent with either apo or holo structures. This discrepancy was attributed to a minor state in equilibrium with the major apo structure. The structure of the minor state was determined with a conjoined rigid-body/torsion-angle simulated annealing refinement (Clore \& Bewley, 2002) and was found to correspond to a partially closed MBP structure. Hence, apo MBP samples high-energy structures that are probably on the trajectory towards the 'bound' conformation, but MBP appears to require interaction with substrate molecules before it can adopt the fully closed structure (Tang et al. 2007).

\subsubsection{Additional examples}

There are several other cases in which NMR experiments have suggested transient populations of functionally important states. Notable examples include HIV protease (Ishima et al. 1999), estrogen receptor DNA-binding domain (Wikström et al. 1999), the C-terminal domain of Sendai virus nucleoprotein (Jensen et al. 2008; Schneider et al. 2015), Abl and Src kinases (Agafonov et al. 2014; Wilson et al. 2015), calmodulin (Gsponer et al. 2008). In addition, it has been suggested that the GPCR $\beta 2 A R$ uses a conformational selection mechanism (Deupi \& Kobilka, 2010), clearly demonstrating that concepts arising from the application of NMR spectroscopy to established model systems are likely to be generally relevant to protein function.

\subsection{Rate limitation in enzymatic catalysis}

Many vital cellular chemical reactions are far too slow to be compatible with any given cellular process. Enzymes are extraordinary bio-catalysts that have evolved to shift the time scale of chemical reactions to a window that is compatible with cellular processes such as cell division. As an example, the enzymatic rate enhancement of orotidine $5^{\prime}$-phosphate decarboxylase is estimated to be an astonishing $10^{17}$ fold (Radzicka \& Wolfenden, 1995). To catalyze chemical reactions, enzymes must accomplish several complex tasks during the reaction (Radzicka \& Wolfenden, 1995), including having a moderate binding affinity for substrates and products to avoid kinetic traps, (Fersht, 2000) extremely tight binding to the transition state compound(s), (Rundqvist et al. 2009) activation of functional groups on the substrate(s), (Wolf-Watz et al. 2004) dehydration of active sites to reduce non-productive side-reactions, and (Rhoads \& Lowenstein, 1968) optimal alignment of substrates to facilitate the chemical transformation into products. In fact all of these steps can be linked to conformational dynamics with differing time scales and amplitudes on the enzyme itself. There are many enzymes whose activity is diffusion limited, i.e. enzymes that are close to perfect (Fersht, 2000). On the other side of the spectrum there are also enzymes with much lower levels of activity, whose catalytic rate constants are far below the limits imposed by diffusion and Brownian motion. In a few such cases, NMR experiments have shown that conformational dynamics constitute the rate limiting step during the enzymatic reaction cycle. For these enzymes, the rate constants for the chemical transformations must be significantly faster than those for the preceding or subsequent conformational changes. Below we will briefly discuss some relevant examples together with the experimental approaches that enabled identification of the rate limiting conformational dynamics.

\subsubsection{Adk}

Adk catalyzes the magnesium-dependent interconversion of AMP and ATP into two ADP molecules. The reaction is fully reversible and the equilibrium constant in the direction of ADP formation is 2.8 in the absence of magnesium (Rundqvist et al. 2009). Since the reaction catalyzed by Adk is reversible, it is possible to study the dynamics of the enzyme during turnover conditions using endogenous substrates. In a study from the Kern laboratory, the dynamics of mesophilic (Escherichia coli) and hyperthermophilic (Aquifex aeolicus) Adk were studied by means of ${ }^{15} \mathrm{~N}$ relaxation dispersion measurements (Wolf-Watz et al. 2004). The substrate concentrations used $(20 \mathrm{mM})$ were well above the $K_{\mathrm{M}}$ values of the enzymes, thus ensuring that the quantified slow motions were of intramolecular nature. Due to the catalytic action of the enzymes, the substrate mixtures for which the dynamics were quantified corresponded to equilibrium mixtures (i.e. AMP, ATP and ADP were all present simultaneously). The process that produced the observed dispersion profiles was identified by showing that the dynamics of the hyperthermophilic variant in the presence of the equilibrium mixture were very similar to those observed in a mixture containing AMP and the non-hydrolysable analogue AMPPNP. It was thus concluded that the detected motion corresponded to opening and closing dynamics in the presence of the bound substrate. It was further found that the microscopic rate constants for domain opening closely matched the catalytic turnover numbers obtained from a coupled ATPase assay (Rhoads \& Lowenstein, 1968). In conclusion, on the basis of converging rate constants between catalytic turnover and conformational dynamics it was inferred that these two Adk variants are rate limited by conformational dynamics (Wolf-Watz et al. 2004). The finding also resolved the question of why thermophilic enzymes often display lower activities than their mesophilic counterparts at ambient temperatures (Zavodszky et al. 1998). In the case of Adk, the entire activity difference between the hyperthermophilic and mesophilic variants at ambient temperatures is explained by the rate constants of conformational dynamics, which are significantly slower in the hyperthermophile (Wolf-Watz et al. 2004). In a recent paper 
from the same laboratory it was shown that the rate constant of the chemical transformation is indeed significantly faster than that of conformational dynamics in Adk (Kerns et al. 2015). As a final note, the use of enzymes from different organisms with different catalytic properties in comparative studies is a powerful way of addressing functional questions while avoiding epistasis (as discussed in more detail below). While it is also possible to investigate functional questions by using point mutations, this carries the risk of changing both the targeted property and additional important properties of the protein.

\subsubsection{RNase A}

Since RNase A catalyzes an irreversible reaction it is not possible to quantify its dynamics during catalytic turnover as was done for Adk. Therefore, the Loria group used a different approach to investigate the motions of RNase A that are relevant to its turnover (Beach et al. 2005). In short, a molecule denoted pTppAp was carefully analyzed and a crystallographic structure of this molecule in complex with RNase A revealed that the specific base and phosphate interactions satisfy the established criteria for a realistic substrate-mimic. Using the approaches discussed above, it was found that the dynamics of a pTppAp:RNase A complex as revealed by relaxation dispersion experiments corresponded to the interconversion of RNase A structures resembling the substrate-free and substrate-bound states. As discussed in the paper (Beach et al. 2005) the rate constant of conformational interconversion of RNase A in the presence of pTppAp is similar to the $k_{\text {cat }}$ value measured for real substrates. Therefore, it was suggested that conformational dynamics are the rate limiting step for RNase A catalysis.

\subsubsection{DHFR}

As discussed above DHFR catalyzes the reduction of DHF to THF through a number of populated intermediates. Quantitative analysis of conformational exchange rate constants from relaxation dispersion profiles provided new insight into the influence of dynamics on the catalytic efficiency of DHFR (Boehr et al. 2006b). It was found that the first-order rate constant for the rate determining release of THF from the E:NADPH:THF complex $\left(12.5 \mathrm{~s}^{-1}\right)$ matched that of the conformational transition between the ground state (E:NADPH:THF) and a high-energy state resembling the E:NADPH complex. This observation has the interesting implication that product release probably occurs from the high-energy state, which is important not just in the context of dynamics but for enzymology in general: it clearly shows that studying ground state structures alone is not always sufficient to understand enzymes' catalytic power. It was also found that the rate constant for hydride transfer was very similar to that for the conformational transition between the ground state of the E:NADP+:THF and an excited state resembling the E:NADP+:folate complex (which was used as a model for the Michaelis complex corresponding to E:NADPH:DHF). Taken together, these results clearly show that the rate constants for conformational dynamics have profound effects on the overall catalytic efficiency of DHFR (Boehr et al. 2006b).

\subsubsection{Tyrosine phosphatases}

Protein tyrosine phosphatases (PTPs) catalyze the cleavage of the tyrosine phosphate monoester (pY) of their protein substrates followed by subsequent hydrolysis of the phosphoenzyme intermediate. Thus, the overall turnover of these enzymes is dictated by

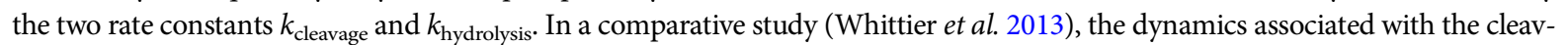
age reaction were quantified for YopH, a virulence factor from Yersinia (Brubaker, 1991), and for PTP1B, a human phosphatase involved in regulating leptin, insulin and epidermal growth factor signaling (Zhang, 2001). Although the two enzymes' substrate interactions are very similar, their $k_{\text {cat }}$ values differ significantly: $700-100 \mathrm{~s}^{-1}$ for YopH and $15-30 \mathrm{~s}^{-1}$ for PTP1B at $T=293 \mathrm{~K}$ (Zhang et al. 1993). The enzymes harbor a ten residue segment known as the WPD loop that contains an aspartic acid which serves as the general acid that protonates the leaving group. The WPD loop undergoes a substantial displacement on going from the open (inactive) to the closed (active) state, which places the general acid in close proximity to the oxygen of the leaving group of the substrate. By using both relaxation dispersion and ${ }^{1} \mathrm{H}-{ }^{15} \mathrm{~N}$-TROSY Hahn-echo experiments (Wang et al. 2003) it was found that the rate constant of WPD loop closure in each enzyme closely matched their rate constants in the presence and absence of a peptide substrate mimic. Thus it appears that the cleavage reaction supported by the action of the general acid is rate limited by the closing motion of the WPD loop (Whittier et al. 2013). The actual chemical reaction, i.e. bond breaking and bond making in the transition state, is much faster (occurring on a femtosecond time scale) than the loop motions that were detected by NMR.

\subsection{Intrinsic motions}

As discussed earlier in this review, an enzyme's ability to catalyze a chemical reaction can be dependent on conformational dynamics and the exchange between stable ground states and high-energy states. As such, it is fundamental to determine whether the time scale and nature of the motions that are relevant for catalysis are determined by substrate binding or are intrinsic properties of the enzyme. Solid state deuterium NMR experiments reported in 1995 showed that that an important loop in triose-phosphate isomerase (TIM) displays an identical frequency of motions in the absence and presence of a 
substrate analogue (Williams \& McDermott, 1995). Moreover, for CypA it has been shown that the motions that occur during catalysis also occur in the apo enzyme in the absence of any substrate (Eisenmesser et al. 2005). Moreover, the frequencies of motions in the presence and absence of substrate molecules correlate with the frequencies associated with catalytic turnover rate constants. These observations suggest that the enzyme motions that are required for catalysis are intrinsic to the enzyme and are 'harvested' to enable productive turnover when the substrate is bound. A similar inference has been made based on the observed motions of RNase A in the absence and presence of the substrate mimic pTppAp (Beach et al. 2005). For protein kinase A (PKA), it has been observed that dynamics enable the sampling of active and inactive states in the absence of substrate but that the time scale of the motions in the apo state does not match with catalysis (Masterson et al. 2011a). Overall it appears that the time scale of motions in apo enzymes may or may not be synchronized with that of catalytic turnover. Nevertheless, all of the enzymes mentioned above clearly sample active conformations in the absence of substrate, and it appears that this is the case for most enzymes.

\subsection{Molecular recognition}

Molecular recognition is a fundamental cornerstone of biology and the large number of ways that proteins can interact with other biological macromolecules or small organic molecules probably reflects its importance. In some cases the interaction interfaces between proteins are sculpted by the interaction energy (as assumed by the induced fit model) and in other cases the interfaces are more or less invariant to association (as assumed by the lock and key model). A wealth of information regarding the structure and energetics of biological interactions is available from x-ray crystallography (Lindgren et al. 2013) and ITC (Kabiri \& Unsworth, 2014). Usually there is an optimal shape and charge complementarity for interaction interfaces (Buckle et al. 1994) and the formation of stable complexes requires extensive burial of solvent-exposed surfaces. For instance, the average burial of surface area in protein-protein complexes is $800 \AA^{2}$ /protein, yielding a total burial of $1600 \AA^{2}$ (Hwang et al. 2008; Janin et al. 2008). Although the basic principles of complex formation in terms of structure and energetics are well-described, there are several mechanistic aspects of molecular recognition that are most readily addressed using solution state NMR spectroscopy. In some instances NMR is the only technique that allows quantitative analysis, such as when quantifying the contribution of conformational entropy to binding affinities (Bingham et al. 2004; Bracken et al. 1999; Diehl et al. 2010; Gagne et al. 1998; Homans, 2005; Stone, 2001; Zidek et al. 1999). Of note is that NMR spectroscopy is uniquely suitable for determining weak binding affinities (i.e. those associated with $K_{\mathrm{D}}$ values that approach the $\mathrm{mM}$ concentration range) such as those between some enzymes and their substrates (Fersht, 2000). In this section we highlight aspects of molecular recognition in which NMR spectroscopy has provided fundamental mechanistic insights.

\subsubsection{Coupled folding/binding}

Coupled folding and binding pathways directly link protein folding and biological function; in such cases, the folding and functional energy landscapes overlap (Olsson \& Wolf-Watz, 2010). Proteins that are unfolded in solution in vitro are often found to be folded in their functional context (i.e. under cellular conditions) because they fully or partially fold on binding to another molecule, which may be another protein, a nucleic acid, a membrane, or a small molecule. Two major pathways for coupled binding and folding have been proposed: folding after binding (which corresponds to an induced fit) and conformational selection. In the folding after binding pathway, the unfolded protein binds to the target and then folds into the final structure, thus utilizing the target molecule as a folding template (Sugase et al. 2007). On the other hand, in the conformational selection pathway a small fraction of the protein is folded when unbound and binding selects the folded form. Intermediate pathways between these two extremes also exist where a small amount of preformed structure might be required for the initial encounter complex. In the following sections we highlight cases where NMR has been used to describe coupled folding and binding processes between proteins and different target molecules such as proteins, lipids, small molecules and nucleic acids.

5.4.1.1 Phosphorylated kinase inducible activation domain (pKID)-KIX. One protein that follows the folding after binding pathway is the pKID of the transcription factor CREB. pKID exists as an unfolded protein in solution in vitro (Radhakrishnan et al. 1997) but folds upon binding to the KIX domain of the CREB binding protein (Parker et al. 1996). The structure of the pKID-KIX complex has been solved by NMR (Radhakrishnan et al. 1997), revealing that the pKID forms two helices and that the C-terminal helix in particular is very well-defined, interacting specifically with KIX (Fig. 5). The binding process was studied using a combination of ${ }^{15} \mathrm{~N}$-relaxation dispersion and chemical shift analysis of the pKID-KIX complex (Sugase et al. 2007). An analysis of chemical shifts of pKID as a function of the relative concentration of KIX showed that most of its amino acid residues experience slow exchange (Sugase et al. 2007) while a subset of residues experience fast exchange at sub stoichiometric KIX concentrations. Relaxation dispersion experiments showed that the protein was present in three different conformations: free protein, an intermediate, and the fully folded complex. This three-state model was chosen instead of a two-state model even though the latter yielded an estimated $K_{\mathrm{D}}$ value that agreed well with ITC data (Zor et al. 2004). These 


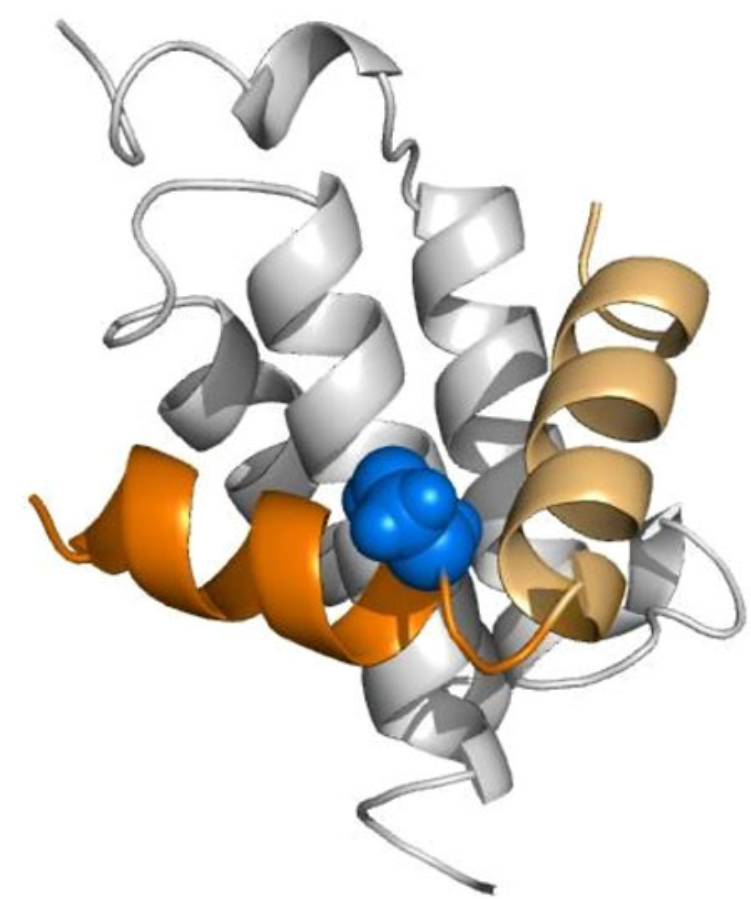

Fig. 5. Coupled folding and binding illustrated with the NMR solution structure of the pKID:KIX complex. The structure of KIX is colored in gray, whereas the two helices of pKID are colored in light- and bright orange, respectively. The location of the phosphorylation site Serine 133 is shown in blue in a side chain representation. This figure was adapted from reference Radhakrishnan et al. (1997).

observations led to the conclusion that an encounter complex is in fast exchange with unbound pKID leading to a folding process that occurs via an intermediate state and in which KIX serves as a folding template (Sugase et al. 2007).

5.4.1.2 Virion protein 16 (VP16)-hTAP/31. The C-terminal (410-490) part of Herpes simplex virion protein 16 (VP16C) contains two transcription activation domains that interact with large proteins in the RNA polymerase II transcription machinery (Jonker et al. 2005). VP16C is unstructured in solution but adopts an alpha-helical conformation upon interaction. This was demonstrated by the appearance of alpha helix-specific NOEs following the addition of a substoichiometric amount of the receptor $\mathrm{hTAF}_{\mathrm{II}} 31$ (Uesugi et al. 1997).

5.4.1.3 SycE-YopE. The effector protein YopE, a protein exported from Yesinia as part of its virulence, interacts specifically with the chaperone SycE. This interaction involves a coupled binding and folding event that is localized to the chaperone binding domain (CBD) region of YopE (Birtalan et al. 2002; Rodgers et al. 2008). The C-terminal catalytic RhoGAP domain is already folded in the absence of SycE and does not undergo any discernible resculpting upon binding to the chaperone. On the other hand, the CBD region shows chemical shifts typical for a random coil when not bound to SycE. The ${ }^{15} \mathrm{~N}-h \mathrm{NOE}$ values for the entire CBD region were all between 0 and $0 \cdot 5$, which suggests a flexible but not fully random coil structure (Rodgers et al. 2008). When YopE binds to SycE, its chemical shifts change in a way that suggests, at least some parts of the CBD-region become alpha-helical. ${ }^{15} \mathrm{~N}-h \mathrm{NOE}$ data show that the CBD-region becomes more rigid upon interaction, which can be attributed both to the formation of secondary structure elements and to the binding of this specific region on the surface of SycE (Rodgers et al. 2008).

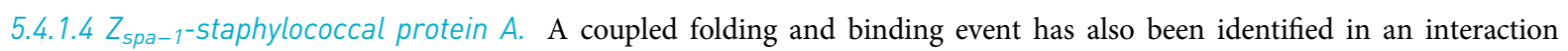
between an engineered protein and its target molecule. The engineered 58 amino acid long protein $Z_{\text {spa-1 }}$, a so called affibody, binds to the original affibody $\mathrm{Z}$ (Wahlberg et al. 2003). This binding event induces a transition from a molten globule state to a well-folded three helix bundle (Wahlberg et al. 2003) similar to the structure of the affibody Z. The ${ }^{1} \mathrm{H}-{ }^{15} \mathrm{~N}-\mathrm{HSQC}$ spectrum of free $\mathrm{Z}_{\text {spa-1 }}$ shows amide proton resonances between 7.5 and $8.5 \mathrm{ppm}$, indicating a flexible structure. In addition, $Z_{\mathrm{spa}-1}$ binds to ANS, and subsequent fluorescence experiments also indicated a molten globule state. However, the dispersion of its amide proton signals increased significantly in the presence of the affibody $\mathrm{Z}$, in parallel with a significant reduction in 1-anilinonaphthalene-8-sulfonate (ANS) fluorescence. Both of these results are consistent with the formation of a folded structure upon interaction (Wahlberg et al. 2003). 
5.4.1.5 Protein-small molecule interactions. Specific segments in proteins can fold upon interaction with small molecules as shown for the interaction between the Trp RNA-binding attenuation protein (TRAP) and tryptophan. TRAP is an undecameric protein that binds to RNA and contributes to the expression control of tryptophan synthesis in many species of bacillus. The binding of TRAP to RNA is regulated by the binding of tryptophan to TRAP. Results from the Forster laboratory (McElroy et al. 2002, 2006) have shown that the binding of tryptophan induces the formation of defined structural elements in TRAP. This was demonstrated by quantifying chemical shift changes and line-broadening caused by the addition of tryptophan to TRAP (McElroy et al. 2002). The affected amino acid residues are not just those in the vicinity of the tryptophan binding site, indicating a general increase in rigidity upon binding (McElroy et al. 2002). In keeping with this conclusion, $\mathrm{H} / \mathrm{D}$ exchange experiments revealed a region of the protein with increased resistance to exchange following tryptophan exposure, again indicating the formation of a more stable structure (McElroy et al. 2006). Among the amino acid residues with increased solvent protection were two acidic residues (Asp37 and Glu40) that have been shown to be involved in RNA recognition (McElroy et al. 2006). Studies on the binding of tryptophan to TRAP using ITC and fluorescence showed that all 11 sites of interaction were independent. Moreover, when the Spolar and Record (Spolar \& Record, 1994) method for computing the number of amino acid residues involved in coupled folding and binding based on measured $\Delta C_{\mathrm{p}}$ values was applied to the TRAP/tryptophan system, the number obtained was very similar to the number of residues exhibiting significant line-broadening in the NMR experiments (McElroy et al. 2006).

5.4.1.6 Protein-DNA interactions. Proteins can fold upon recognition of nucleic acids as was shown for the N-terminal 175 amino acid residues in the DNA binding domain of the Brinker protein (Brk). A ${ }^{1} \mathrm{H}_{-}{ }^{15} \mathrm{~N}$ HSQC spectrum showed that this protein is unstructured in solution. However, upon binding to DNA about $50 \%$ of the ${ }^{1} \mathrm{H}-{ }^{15} \mathrm{~N}-\mathrm{HSQC}$ signals exhibit dispersion behavior consistent with that expected for a folded protein (Cordier et al. 2006). Subsequent assignment showed that the dispersed signals belonged to the C-terminal half of the domain, and the corresponding ${ }^{15} \mathrm{~N}-h \mathrm{NOE}$ values indicated a significant gain of order in the same region (Cordier et al. 2006). MD simulations of the binding of Brinker to DNA suggest that this occurs via an induced fit reaction (Qin et al. 2011).

5.4.1.7 Protein-lipid interactions. A recent example of an interaction between a protein and a lipid membrane is the interaction between a domain of $\mathrm{YscU}$, one of the proteins that constitutes the type three secretion system in Yersinia-bacterial, and a lipid bilayer. YscU is a membrane protein consisting of two domains, a membrane integral domain $\left(\mathrm{Ysc}_{\mathrm{N}}\right)$ and a soluble domain $\left(\mathrm{Ysc}_{\mathrm{C}}\right)$. The linker between the domains is unstructured in solution. However, it incorporates a motif with positively charged amino acids separated by two or three other amino acids, so if it were to form an alpha-helix it would have a strongly positively charged face (Weise et al. 2014). ${ }^{13} \mathrm{C}$ chemical shift perturbations observed at binding of the linker to SDS-micelles and ${ }^{3} \mathrm{~J}_{\mathrm{HNHA}}$-couplings both suggested that the linker forms an alpha-helix when in contact with negatively charged lipids. The folding event was also confirmed in more realistic membrane models by CD spectroscopy. Point mutations that caused some of these positively charged amino acids to be replaced with alanine strongly reduced membrane interaction and also adversely affected effector protein secretion (Weise et al. 2014) thus confirming the biological role of the protein-membrane interaction.

\subsubsection{Cracking or 'order-disorder-order' transitions}

It has been postulated that one way of increasing the rate constants of functional transitions in proteins is by 'cracking', i.e. the unfolding of localized polypeptide segments in the transition state structure (Hyeon \& Onuchic, 2007; Miyashita et al. 2003). This postulate builds on a notion from energy landscape theory (Frauenfelder et al. 1991, 2009; Onuchic \& Wolynes, 1993) that proteins constantly fluctuate between states in a stochastic way. During a functional transition, a protein may build up strain that is released through partial unfolding once it has exceeded a certain threshold. This unfolding event may then cause a gain in conformational entropy that can reduce the free energy barrier for the transition (Whitford, 2013). In essence the idea is that a protein that populates two different and completely folded structural states in inactive and active conformations can transiently populate a partially disordered state in the transition state. In analogy to such 'disorder-order' transitions, cracking models can be formulated in terms of an 'order-disorder-order' event (Olsson \& Wolf-Watz, 2010). Cracking is inherently difficult to probe experimentally but there are cases when its occurrence has been inferred from simulations. Proteins suggested to undergo cracking include Adk (Whitford et al. 2007, 2008), epidermal growth factor receptor (Shan et al. 2013), calmodulin (Tripathi \& Portman, 2009) and influenza hemagglutinin (Lin et al. 2014). Here we will briefly summarize the computational results for Adk and cracking in relation to a reaction coordinate linking open (inactive) and closed (active) states. Cracking was inferred from simulations in which the system was allowed to follow low frequency collective modes (Miyashita et al. 2003) and from coarse grained simulations using structure-based potentials (Taketomi et al. 1975; Whitford et al. 2007). A converging picture emerged from these studies which suggested that, segments in which cracking were likely could be found in both of the nucleotide-binding domains of Adk. A fundamental requirement for cracking to 
occur is that the local stabilities of the relevant polypeptide sequences must be low enough to allow strain release via local unfolding. This appears to be the case for Adk, for which NMR-based H/D exchange experiments have clearly demonstrated that the flexible nucleotide binding domains are significantly less stable than the protein is globally (Rundqvist et al. 2009). Thus, the underlying energetics required to enable cracking are present in Adk. In an experimental approach designed to test the cracking model (Olsson \& Wolf-Watz, 2010) point mutations in Adk were designed to destabilize a segment of the ATPlid that was suggested to crack during the enzyme's functional transition. NMR monitoring revealed that the mutants were folded and adopted the correct structural motifs at the extremes of the reaction coordinate (i.e. in the open and closed states). However, the equilibrium constant for the folding/unfolding reaction was shifted more towards the unfolded state in the mutated variants. The mutants' functionality was evaluated and benchmarked against the wild type in two experiments: (i) an ATP binding affinity assay probed by ITC, and (ii) an enzymatic activity assay probed using a coupled ATPase assay (Rhoads \& Lowenstein, 1968). Notably the mutated and destabilized Adk variants displayed a fifty fold improved ATP binding affinity ( $K_{\mathrm{D}}$ values decreased from 50 to $\left.1 \mu \mathrm{M}\right)$. This is a remarkable result since in general destabilization of a protein is expected to impair functionality. The result is fully consistent with the idea that the mutations act to push the enzyme along the free energy surface in a way that is coupled to conformational change, and that the transition state structure is more disordered than the stable ground states. A related increase in binding affinity in response to a perturbation of native contacts has been observed for the binding of a peptide to the fibronectin extradomain B via a local unfolding mechanism (Yu et al. 2014). Since Adk is rate limited by the opening of its substrate binding domains, these mutations should in fact reduce the enzyme's catalytic efficiency (with the assumption that the free energy surfaces are robust to the mutations). This matches experimental observations: the variants retain only approximately $3 \%$ of wild type activity. Taken together, the experimental observations are fully consistent with the cracking model but do not provide definite evidence of its validity.

\subsubsection{Coupled unfolding/binding}

As discussed above, coupled folding and binding appears to be a fundamental concept in molecular recognition. Having established this model, it is not farfetched to assume that coupled unfolding and binding reactions may also be relevant in biological recognition processes. This has indeed recently been shown in an elegant paper by Suh and co-workers (Yu et al. 2014). The authors show in a very convincing manner that a $\beta$-strand in the fibronectin extra domain B (EDB) detaches from the core of the protein structure upon binding of a class of high-affinity peptides called aptides (Kim et al. 2012). In the bound complex, the detached $\beta$-strand is replaced with a strand from the aptide. The work rests on a firm structural basis and the structures of both ligand-free EDB and a complex between EDB and an aptide were solved by NMR spectroscopy. Perhaps the most notable result is that the fast ps-to-ns mobility is significantly increased in the complex with the detached $\beta$-strand. The mechanism put forward by the authors thus involves a coupled local unfolding and binding event. The final result, i.e. a $\beta$-strand donation from the ligand to the protein, is fundamentally related to the 'donor strand complementation' model that was discovered for chaperone-adhesin complexes in bacterial pili (Choudhury et al. 1999; Sauer et al. 1999). In a broader sense, it seems that cooperative folding reactions are fundamental in molecular recognition and that several flavors of folding are utilized, as exemplified by systems making use of 'coupled folding-binding', 'coupled unfoldingbinding' and 'cracking' mechanisms for recognition of their respective targets. Since the cooperative folding reaction is evolutionarily robust, it comes as no surprise that it is used to add functionality to proteins.

\subsubsection{Folding by phosphorylation}

Most binding events leading to folding are driven by binding energetics arising from non-covalent interactions. The intrinsically disordered protein 4E-BP2 represents a notable exception in that its folding is induced by phosphorylation. The phosphorylation-driven folding of 4E-BP2 was detected on the basis of differences in ${ }^{15} \mathrm{~N}$ - $h \mathrm{NOEs}$ and the dispersion of chemical shifts observed in a ${ }^{1} \mathrm{H}-{ }^{15} \mathrm{~N}-\mathrm{HSQC}$ spectrum for phosphorylated and non-phosphorylated versions of the protein (Bah et al. 2015). Since the protein's role is to bind to eIF4E in order to suppress cap-dependent translational initiation, the strength of the interaction between 4E-BP2 and eIF4E was evaluated using ITC. These titration experiments showed a significant decrease in binding affinity for the phosphorylated form, with $K_{\mathrm{D}}$ increasing from 3 to $12320 \mathrm{nM}$. This suggests that the phosphorylation event suppresses binding and thereby activates cap-dependent translation (Bah et al. 2015).

\subsubsection{Conformational entropy}

The energetics underlying molecular recognition are described by the standard Gibbs free energy, $\Delta G^{\circ}=\Delta H^{\circ}-T \Delta S^{\circ}$, and the relationship between the standard free energy and the equilibrium constant, $\Delta G^{\circ}=-R T \ln K_{\mathrm{D}}$. Both the enthalpic $\left(\Delta H^{\circ}\right)$ and entropic $\left(\Delta S^{\circ}\right)$ components can be obtained from ITC experiments. However, these components reflect total changes in the energetics of the protein, the ligand and the solvent (including any co-solvents such as ions). Thus a fundamental problem in 
understanding energetics data obtained by ITC is the difficulty of deconvoluting the individual components of the system of interest. The entropic component is of particular interest and since the overall entropy contains a large desolvation term it is challenging to quantify the contribution from protein conformational entropy to a binding event. However, there are NMR approaches that can be used to estimate changes in a protein's conformational entropy based on analyses of order parameters (Akke et al. 1993a; Li \& Bruschweiler, 2009; Wand, 2001; Yang \& Kay, 1996). In the following section we describe some cases where estimates of protein conformational entropy provided new insight into molecular recognition.

5.4.5.1 Ligand binding. In a study on the energetics of carbohydrate binding to the carbohydrate recognition domain of Galectin-3, Akke and co-workers made the surprising discovery that protein conformational fluctuations on the ps-to-ns time scale, probed with ${ }^{15} \mathrm{~N}$ (Akke et al. 1993b; Farrow et al. 1994b; Hansen et al. 2007, 2009) and ${ }^{2} \mathrm{H}$ spin relaxation (Millet et al. 2002), are amplified in the ligand bound state relative to unbound states (Akke, 2012; Diehl et al. 2010). This finding is at large with the, perhaps more intuitive, quenching of fast time scale motions in protein-ligand complexes as observed for instance for Adk (Shapiro et al. 2000). The changes in the fast dynamics of Galectin-3 were translated into relative changes in protein conformational entropy driven by ligand binding by using a previously developed formalism (Akke et al. 1993a; Li \& Bruschweiler, 2009). Notably the changes in conformational entropy strongly favor carbohydrate binding and the magnitude of this term is comparable with the enthalpic component derived from ITC. This surprising result illustrates that fundamental aspects of the entropic contribution to binding remain to be explored and clarified. The analysis here is based on the assumption that fluctuations of individual residues are uncorrelated (Akke et al. 1993a) and therefore the results represents an upper limit on the entropy for the degrees of freedom that are probed (it should be noted that correlated motions on slower time scales can be studied using NMR (Bouvignies et al. 2005; Lundström et al. 2005)). The contributions of conformational entropy to ligand binding have also been evaluated for a number of other proteins including calmodulin (Frederick et al. 2007; Marlow et al. 2010), protein kinase A (Masterson et al. 2010), and mouse major urinary protein (Zidek et al. 1999).

5.4.5.2 Negative binding cooperativity. Negative binding cooperativity cannot be explained with the MWC model (Monod et al. 1965), but the sequential or Koshland-Nemethy-Filmer model can account for this effect (Koshland et al. 1966). As an alternative it was proposed in 1984 (Cooper \& Dryden, 1984) that entropic contributions (manifested as protein dynamics) may represent another way in which negative binding cooperativity has evolved. Estimates of changes to conformational entropy following ligand binding to glycerol-3-phosphate:CTP transferase (GCT) (Stevens et al. 2001) and the catabolite activator protein (CAP) (Popovych et al. 2006) have now confirmed this early prediction. Here we describe the CAP example in some detail. CAP is a symmetric homodimer with each monomer having 209 amino acid residues. Its DNA binding affinity is modulated by binding of two molecules of cAMP (Harman, 2001). Binding of cAMP to CAP causes negative cooperativity and the binding constants for the first and second equivalent of cAMP are 0.04 and $4 \mu \mathrm{M}$, respectively (Popovych et al. 2006). From a structural standpoint system is well-characterized and the structures of apo CAP (Popovych et al. 2009), CAP-cAMP 2 loaded (Passner et al. 2000), and CAP-cAMP 2 -DNA (Schultz et al. 1991) have been reported. Briefly, the DNA recognition helices adopt a DNA binding incompetent state in the apo protein; upon cAMP binding, the orientation of these helices changes and they become poised for DNA binding. In a study from the Kalodimos laboratory it was found that the negative binding cooperativity observed for successive cAMP binding events to CAP is, at least in part, entropic in origin (Popovych et al. 2006). This conclusion was drawn after having quantified ps-to-ns motions in the protein by analyzing the spectral density function in three different states: (i) apo CAP, (ii) CAP-cAMP and (iii) CAP-cAMP ${ }_{2}$. It was possible to selectively populate the cAMP and $\mathrm{CAMP}_{2}$ bound states since the binding affinities for the binding of the first and second cAMP equivalent differ by two orders of magnitude. Notably, the ps-to-ns motions are very similar in the apo and cAMP bound states. However, upon binding of the second cAMP molecule there is a drastic reduction of these motions across the entire dimer. These effects were further quantified by evaluating the backbone order parameters, $S^{2}$, and using them to estimate the associated changes in conformational entropy. In summary, at least a part of the negative binding cooperativity observed for cAMP binding to CAP is explained by a decrease in conformational entropy that is exclusively associated with the binding of the second cAMP equivalent. As a final note, it appears that conformational entropy can also be relevant for positive binding cooperativity since it has been observed that successive binding of $\mathrm{Ca}^{2+}$ to calbindin $\mathrm{D}_{9 \mathrm{k}}$ is associated with significant changes in fast time scale motions (Mäler et al. 2000).

\subsubsection{Encounter complexes}

The rapid and specific association of protein complexes is a central component of many biological processes. For diffusioncontrolled binding, the basal association rate constants are restricted by diffusion and the geometric constraints of binding surfaces. The binding process can be further slowed down through isomerization reactions on the protein (such as 
conformational changes) that occur after the initial encounter (Schreiber, 2002). A minimal reaction for the association between two proteins, $\mathrm{A}$ and $\mathrm{B}$, is then:

$$
A+B \underset{k_{-1}}{\stackrel{k_{1}}{\longrightarrow}} A B_{k_{-2}}^{\stackrel{k_{2}}{\longrightarrow}} A B^{* *} \underset{k_{-3}}{\stackrel{k_{3}}{\longrightarrow}} A B
$$

where $\mathrm{AB}^{*}$ is an encounter complex, $\mathrm{AB}^{* *}$ is an intermediate that is obtained following the isomerization of $\mathrm{AB}^{*}$, and $\mathrm{AB}$ is the stereospecific complex. Typical association rate constants $\left(k_{1}\right.$ in the above equation) are in the range of $10^{5}-10^{9} \mathrm{M}^{-1} \mathrm{~s}^{-1}$ and the absolute value can be strongly dependent on electrostatic interactions and ionic strength (Schreiber \& Fersht, 1996). When a non-specific encounter complex has been formed, a protein can engage in a 2D diffusion process that will eventually lead to the formation of the stereospecific complex. Since encounter complexes consist of several transient and weakly bound conformations, they are silent to many structural biology techniques. However, they can be studied using NMR because weak binding $\left(K_{\mathrm{D}}\right.$ values on the order of $\left.\mathrm{mM}\right)$ are readily detected. A fundamental question relates to the structure of the encounter complex $\left(\mathrm{AB}^{\star}\right)$; as expected, it is most adequately described as an ensemble of conformers. Below we briefly review two cases in which NMR has been used to obtain detailed information on encounter complexes.

5.4.6.1 The N-terminal domain of enzyme / (EIN) and phosphocarrier protein. In a PRE-based study (Clore, 2008), Clore and co-workers characterized an encounter complex ensemble $\left(\mathrm{AB}^{\star}\right.$ in Eq. (13)) between $\mathrm{EIN}$ and the phosphocarrier protein (HPr). This was done by assuming that the binding reaction involves fast exchange such that observed PRE values are population weighted averages. Three sites outside the EIN interaction surface on HPr were individually replaced with cysteines and these sites were conjugated to EDTA through a disulfide linkage to yield a cysteaminyl-EDTA-Cys adduct. The adducts were then chelated with $\mathrm{Mn}^{2+}$ or $\mathrm{Ca}^{2+}$ to obtain paramagnetic and diamagnetic states, respectively. A comparison of the observed intramolecular PRE rates of the backbone amides $\left({ }^{1} \mathrm{H}_{\mathrm{N}}-\Gamma_{2}\right)$ of EIN with rate constants back-calculated from the stereospecific EIN-HPr (Garrett et al. 1999) complex revealed regions with large discrepancies. These discrepancies were interpreted as evidence of a sparsely populated minor species that exists in rapid exchange with the final stereospecific complex. A structural ensemble of the minor states was generated with restrained rigid-body simulated annealing refinement (Schwieters et al. 2006) using ${ }^{1} \mathrm{H}_{\mathrm{N}}-\Gamma_{2}$ relaxation rates from three HPr variants simultaneously. After cross-validation, it was concluded that the optimal ensemble size was 10-20 conformers with a population of minor species below approximately $10 \%$. The distribution of non-specifically bound HPr molecules on the surface of EIN was found to be non-uniform and qualitatively correlated with the latter protein's negative electrostatic potential isosurface. Moreover, HPr predominantly uses its positively charged regions to interact with the negative EIN surface, highlighting the importance of charge-charge interactions for encounter complex formation (Schreiber \& Fersht, 1996).

5.4.6.2 Cytochrome $\mathrm{c}(\mathrm{C} \mathrm{C})$ and cytochrome $\mathrm{c}$ peroxidase (CCP). Electron transport complexes require the generation of stereo-specific complexes to facilitate electron transfer, but these complexes must be sufficiently weak and transient to allow high turnover rate constants (Bashir et al. 2011). Yeast iso-1-Cc and yeast CcP are arguably the paradigm systems for studies on intermolecular electron transfer (Volkov et al. 2006). The crystallographic structure of the stereospecific complex has been solved (Pelletier \& Kraut, 1992) and there are indications that the complex is dynamic (Jeng et al. 1994; Northrup et al. 1988). In two contributions from the Ubbink laboratory, the surface probed by Cc in the encounter complex with Ccp was defined using PREs (Bashir et al. 2010; Volkov et al. 2006). Paramagnetic spin labels were introduced on CcP through attachment of $S$-(1-oxyl-2,2,5,5-tetramethyl-2,5-dihydro-1H-pyrrol-3-yl)methyl methanesulfonothioate (MTSL) to engineered cysteines, and the PRE enhancements on Cc were converted into distance restraints (Gillespie \& Shortle, 1997a). The measured PREs were analyzed in light of the stereospecific complex (Pelletier \& Kraut, 1992) and an encounter-complex ensemble generated from Monte Carlo simulations (Bashir et al. 2010) and it was found that both these structural descriptions were required to yield a satisfactory account of the experimental data. However, as noted by the authors, sampling encounter complexes in this way arguably reflects a poor formulation of the originally posed question and can only yield truly conclusive results in cases where no interaction occurs at all (Schilder et al. 2014). Nevertheless, it was found that the stereospecific complex and encounter complexes were occupied for 70 and $30 \%$ of the complex's total lifetime, respectively. Moreover, in the encounter complexes Cc samples about $15 \%$ of the surface area of CcP surrounding the stereo-specific binding site (Bashir et al. 2010). In agreement with the EIN-HPr study, it was found that, electrostatics are the most important forces in the formation of encounter complexes.

\subsection{Chaperonins}

\subsubsection{GroEL}

Chaperonins, a sub-class of the larger group of molecular chaperones, are assembled into cylindrical, stacked ring structures with large internal cavities that can encapsulate substrate or client proteins (Braig et al. 1994). These proteins are found in all 
cell types in all organisms where they facilitate folding, assembly and transport. The most extensively studied chaperonin, both structurally and mechanistically, is the GroEL-GroES machinery (Horwich \& Fenton, 2009). GroEL is a 780-kDA assembly consisting of two stacked cylindrical units, each of which is built up from seven identical 56-kDa subunits. Encapsulation and release of substrate proteins is accomplished through a series of ATP-driven conformational changes to GroEL together with recruitment of the co-chaperone GroES (Horwich \& Fenton, 2009). Questions under considerable debate in the scientific literature are: (1) how GroEL interacts with substrate proteins and (2) the mechanism of GroEL-mediated protein folding. As discussed below, NMR has provided important insights into these challenging questions.

5.5.1.1 Substrate binding. In a study from the Gierasch laboratory based on transferred nuclear Overhauser effects it was found that a 13 amino acid residue peptide derived from the N-terminus of rhodanase, although disordered in solution, adopted an amphiphatic alpha-helical conformation when bound to GroEL (Landry \& Gierasch, 1991). The choice of the peptide was based on the fact that GroEL facilitates in vitro refolding of rhodanase (Mendoza et al. 1991). In a follow-up study from the same laboratory a larger set of peptides with varying sequence motifs were investigated and it was found that peptides can adopt other conformations than alpha helical (such as $\beta$-conformations) while bound to GroEL. It was further found that an important determinant for GroEL binding is presence of a hydrophobic surface (Wang et al. 1999). As a comparison, well-ordered $\beta$-hairpin (Chen \& Sigler, 1999) and extended conformations (Buckle et al. 1997) have been found from x-ray crystallography but the relative proportions of these bound conformations cannot be judged from analyses of protein crystals. In a comprehensive relaxation-based study of the interaction between the polypeptide amyloid $\beta$ (A $\beta 40)$ and GroEL it was found that $\mathrm{A} \beta 40$ remains dynamically and structurally disordered while bound to the chaperonin (Libich et al. 2013). The primary sites of interaction were found to be in two hydrophobic segments that overlap with a predicted GroEL consensus sequence (Stan et al. 2006). It was further suggested that A $\beta 40$ exchanges between several sites within the GroEl cavity and that its binding is of multivalent nature. The methodological arsenal applied in this study consisted of: (1) lifetime line-broadening analysis (Fawzi et al. 2010), (2) DEST (Fawzi et al. 2011, 2012), (3) relaxation dispersion experiments (Loria et al. 1999; Tollinger et al. 2001) and (4) quantitative analysis of exchange-induced chemical shifts (Skrynnikov et al. 2002). It is undeniably relevant to address the properties of intact proteins while bound to GroES and such efforts require specialized NMR techniques that permit studies of very large systems. A bound complex between initially chemically denatured DHFR and GroEL has been characterized with NMR (Horst et al. 2005). In order to enable studies of these large assemblies, the authors acquired $2 \mathrm{D}\left[{ }^{1} \mathrm{H},{ }^{15} \mathrm{~N}\right]-\mathrm{TROSY}, 2 \mathrm{D}$ $\left[{ }^{1} \mathrm{H},{ }^{15} \mathrm{~N}\right]$-CRIPT-TROSY and 2D $\left[{ }^{1} \mathrm{H},{ }^{15} \mathrm{~N}\right]$-CRINEPT-HMQC- $\left[{ }^{1} \mathrm{H}\right]$-TROSY experiments (Fiaux et al. 2002; Pervushin et al. 1997; Riek et al. 2002). Build-up of magnetization experiments using insensitive nuclei enhanced by polarization transfer (INEPT) or cross-correlated relaxation-induced polarization transfer (CRIPT) transfer elements indicated that bound DHFR exhibits internal mobility on both the ps-to-ns and the $\mu$ s-to-ms time scales. In conclusion it was suggested that bound DHFR lacks stable tertiary contacts and may best be described as a dynamic ensemble of disordered conformers. Observations of the interaction between Hsp90 and the DNA binding domain of p53 suggest that the dynamic and multivalent interaction observed between chaperonins and full length proteins is also general to the larger family of molecular chaperones (Bukau \& Walker, 1989; Bukau et al. 2006; Ellis, 1996; Miller et al. 2015; Richter \& Buchner, 2001). This inference is made on the basis of hydrogen-exchange measurements and line-broadening, which suggest that the DNA binding domain of p53 adopts an ensemble of conformers (possibly a molten globule state) in complex with Hsp90 (Park et al. 2011).

5.5.1.2 GroEl mediated protein folding. There exist different models that can explain the catalytic folding of proteins by GroEL. Here we discuss the results from two benchmark publications that reported hydrogen/deuterium NMR exchange experiments which provided support for the hypothesis that GroEL acts according to the 'annealing' (Zahn et al. 1996) or 'Anfinsen cage' (Horst et al. 2007) models. It should be stressed that these models are not mutually exclusive but merely describe two distinct aspects of GroEL-stimulated protein folding.

Bacillus amyloliquefaciens ribonuclease (barnase) is a protein that has been thoroughly characterized with respect to amide protein exchange and thus serves as a suitable model for studying the influence of GroEL on these exchange processes. If GroEL functions by catalyzing unfolding (in order to allow and promote subsequent correct refolding) it can be postulated that the rate constants for amide protons whose exchange is dominated by global unfolding should increase. The amide proton exchange processes of barnase can be clustered into two sets; 16 amide protons exchange predominantly through local fluctuations whereas 15 exchange only from the fully unfolded state (i.e. via global unfolding) (Clarke et al. 1993). In presence of catalytic amounts of GroEL there is a notable change to the amide-protein exchange pattern where the most prominent feature is that the time scale for residues exchanging through global unfolding is increased by at least one order of magnitude (Zahn et al. 1996). Moreover, the measured rate constants approach a unified and limiting value, indicating a switch from the EX2 to the EX1 limit of the exchange process in the presence of GroEL. In the EX1 limit, the exchange is given by the rate constant for 
formation of an unprotected state (here unprotected means 'in the absence of hydrogen bonds'). This rate constant can then be interpreted as the unfolding rate constant. In contrast, the rate constants for residues that exchange through local fluctuations are similar regardless of GroEL's presence or absence. In summary, these experiments support an 'annealing' mechanism in which GroEL would have the potential to correct the misfolding of proteins by catalyzing their unfolding. The data are also fully consistent with the 'Anfinsen cage' model where the folding process is promoted in the cavity formed by GroEL. In the 'Anfinsen cage' model the sole determinant of the folding process is the primary sequence of the polypeptide (Ellis, 2003).

Strong support for the description of the folding chamber in GroEL as an 'Anfinsen cage' was obtained in an extensive study on the folding of human DHFR (hDHFR) (Horst et al. 2007). In the presence of ATP and methotrexate (Mtx), the temporal evolution of resistance to H/D exchange was virtually identical for hDHFR free in solution and inside the GroEL cavity (Horst et al. 2007). In brief, the experimental protocol used to monitor $H / D$ exchange over time involved (1) denaturation of ${ }^{15} \mathrm{~N}$-labelled hDHFR with guanidine hydrochloride, (2) dilution into buffer with GroEL, (3) addition of GroES, ATP and Mtx (at the start of the experiment), (4) a variable time delay, (5) the addition of ten volumes of $\mathrm{D}_{2} \mathrm{O}$, (7) incubation until the reaction was completed, (8) acquisition of $2 \mathrm{D}{ }^{1} \mathrm{H}-{ }^{15} \mathrm{~N}-\mathrm{HSQC}$ spectra and (9) evaluation of the build-up of NMR peak intensities. The experimental design with a variable time delay is favorable and in contrast to more traditional approaches (where protonated protein is diluted directly into $\mathrm{D}_{2} \mathrm{O}$ ) it is not dependent on the acquisition time of the read-out experiments; the dead time is limited to about $15 \mathrm{~s}$.

\section{Outlook}

NMR spectroscopy has developed into a mature technique that is being used to make important contributions to our understanding of complex biochemical and biophysical research questions. Although the initial and arguably most notable contribution in the application of NMR to protein chemistry was the development of techniques for protein structure determination (Wuthrich, 2001), today NMR is the most important tool for quantification of protein dynamics at atomic resolution (Boehr et al. 2006a; Ishima \& Torchia, 2000; Kay, 1998; Kern et al. 2005; Palmer, 2004; Palmer et al. 2001). As described earlier in this review, it has been of outstanding importance in many groundbreaking discoveries in protein chemistry. It is thus interesting to consider what directions NMR research might move in to ensure that this technique remains a cornerstone of contemporary protein research. It is already evident that NMR often is used together with other techniques in an integrated fashion to solve demanding research questions. One development with great potential is the integration of NMR studies with genetic approaches to reveal fundamentally new aspects of protein function. This has already been accomplished in a few cases (Lee et al. 2014), for example in the discovery of a coupling between an irreversible dynamic event (i.e. protein 'homo dimer' dissociation) and effector secretion linked to Yersinia pathogenicity (Frost et al. 2012). One fundamental advantage of genetic approaches is that new aspects of protein function can be found in a 'model-independent' manner that requires no prior hypothesis as long as the experimental setup is well-conditioned.

Comparative studies on related proteins originating from different organisms (Bhabha et al. 2013; Holliday et al. 2015; Robic et al. 2003) are powerful tools for addressing research questions related to specific protein properties such as enzymatic $k_{\text {cat }}$ values. The strength of the approach is that the sequences can be treated as mutations that are designed by evolution and properties such as $3 \mathrm{D}$ structure and stability can be similar such that a property of interest can be addressed exclusively. This approach was used successfully in a comparative study of mesophilic and hyperthermophilic Adk that focused on the linkage between dynamics and enzymatic $k_{\text {cat }}$ values (Wolf-Watz et al. 2004). A related approach that holds promise for future studies involves the application of ancestral reconstruction while posing mechanistic questions. An important benefit of this approach is that it avoids problems associated with epistasis (i.e. the effect of surrounding amino acid residues on protein properties), enabling a property of interest to be addressed exclusively (Dellus-Gur et al. 2015). For instance it was shown with this approach that the cancer drug Gleevec binds more strongly to Abl than to Src kinases due to the magnitude of the equilibrium constant of an induced fit conformational rearrangement (Wilson et al. 2015).

There is increasing interest among biophysicists in studying proteins in their real context, i.e. inside living cells. 'In-cell' and 'in-lysate' NMR spectroscopy (Danielsson et al. 2013, 2015; Serber et al. 2005, 2007; Smith et al. 2013) have developed to a point where new aspects of protein function can be revealed (Banci et al. 2013). For instance in a recent study Selenko and co-workers developed an NMR-based methodology that enables quantification of the temporal phosphorylation and de-phosphorylation of short protein segments in cell lysates (Thongwichian et al. 2015). With this approach the efficacy of kinase drugs can be studied for many kinase targets simultaneously and also under near-cellular conditions. It is more than likely that 'in-cell' NMR will continue to develop such that new insights into protein function in a cellular context will be revealed. Perhaps the greatest challenge to overcome in such studies will be the design of robust experiments that enable conclusions to be drawn even in the presence of the complicated conditions represented by cells or lysates. 
There has been significant progress in development of structure calculation protocols that take chemical shifts as input (Cavalli et al. 2007; Sgourakis et al. 2011; Shen \& Bax, 2015; Shen et al. 2010; Wishart et al. 2008). Together with the further development of spin relaxation techniques and isotope labeling strategies it is likely that protein dynamics will also be possible to study from chemical shifts. There has been development in this direction (Lehtivarjo et al. 2012; Li \& Brüschweiler, 2011; Markwick et al. 2010; Robustelli et al. 2012) and the time averaging of chemical shifts in these methods is based on molecular dynamics simulations, which means that fast time scale motions can be addressed. As an example, Vendruscolo and co-workers developed a methodology that enabled characterization of a conformational equilibrium in Rnase A by including chemical shifts as restraints in molecular dynamics simulations (Camilloni et al. 2012, 2013). Since most NMR projects generate chemical shift assignments, further development will enable quantification of dynamics in increasingly large and complex systems.

The introduction of polarization techniques (Riek et al. 2002) and TROSY (Pervushin et al. 1997) spectroscopy (Tugarinov et al. 2003) has made it possible to study the structure and dynamics of very large proteins and protein assemblies by NMR (Fiaux et al. 2002; Gelis et al. 2007; Mainz et al. 2013; Rudiger et al. 2002; Saio et al. 2014; Sprangers et al. 2005, 2007; Sprangers \& Kay, 2007; Tugarinov et al. 2004). Ongoing development in these areas will undoubtedly enable the investigation of still larger and more complex systems, ensuring that NMR is no longer limited to smaller proteins and assemblies.

And finally, although not covered in this review, there have been tremendous developments in solid state NMR spectroscopy both with respect to protein structure determination (Colvin et al. 2015; Lendel et al. 2014; Marchanka et al. 2015; Park et al. 2012; Radoicic et al. 2014) and the quantification of protein dynamics (Lewandowski, 2013; Ullrich \& Glaubitz, 2013). In summary, we conclude that the future prospects for NMR-driven protein research are bright and we can expect many breakthrough discoveries based on this versatile and powerful technique.

\section{Acknowledgements}

This work was financially supported by the Swedish Research Council to M. W.-W, and a postdoctoral fellowship from the Deutsche Forschungsgemeinschaft to M. K. (KO4687/1-1).

\section{References}

Abragam, A. (1961). Principles of Nuclear Magnetism. Oxford: Oxford Scientific Publications.

Ådén, J., Verma, A., Schug, A. \& Wolf-Watz, M. (2012). Modulation of a pre-existing conformational equilibrium tunes Adenylate kinase activity. Journal of the American Chemical Society 134, 16562-16570.

Ådén, J. \& Wolf-WATZ, M. (2007). NMR identification of transient complexes critical to adenylate kinase catalysis. Journal of the American Chemical Society 129, 14003-14012.

Agafonov, R. V., Wilson, C., Otten, R., Buosi, V. \& Kern, D. (2014). Energetic dissection of Gleevec's selectivity toward human tyrosine kinases. Nature Structural \& Molecular Biology 21, 848-853.

AкKe, M. (2012). Conformational dynamics and thermodynamics of protein-ligand binding studied by NMR relaxation. Biochemical Society Transactions 40, 419-423.

Akke, M., Brúschweiler, R. \& Palmer, A. G. (1993a). NMR order parameters and free energy: an analytical approach and its application to cooperative Ca2+ binding by Calbindin D9k. Journal of the American Chemical Society 115, 9832-9833.

AKKe, M. \& PALMER, A. G. (1996). Monitoring macromolecular motions on microsecond to millisecond time scales by R1 $\rho-\mathrm{R} 1$ constant relaxation time NMR spectroscopy. Journal of the American Chemical Society 118, 911-912.

Akke, M., Skelton, N. J., Kordel, J., Palmer, A. G. III \& Chazin, W. J. (1993b). Effects of ion binding on the backbone dynamics of calbindin D9k determined by $15 \mathrm{~N}$ NMR relaxation. Biochemistry 32, 9832-9844.

Almond, A. \& Axelsen, J. B. (2002). Physical interpretation of residual dipolar couplings in neutral aligned media. Journal of the American Chemical Society 124, 9986-9987.

Anthis, N. J. \& Clore, G. M. (2015). Visualizing transient dark states by NMR spectroscopy. Quarterly Reviews of Biophysics 48, 35-116.

Arora, K. \& Broокs, C. L. (2007). Large-scale allosteric conformational transitions of adenylate kinase appear to involve a population-shift mechanism. Proceedings of the National Academy of Sciences of the United States of America 104, 18496-18501.

Bah, A., Vernon, R. M., Siddiqui, Z., Krzeminski, M., Muhandiram, R., Zhao, C., Sonenberg, N., Kay, L. E. \& Forman-Kay, J. D. (2015). Folding of an intrinsically disordered protein by phosphorylation as a regulatory switch. Nature 519, 106-109.

Bai, Y.W., Milne, J.S., Mayne, L. \& Englander, S. W. (1993). Primary structure effects on peptide group hydrogen-exchange. Proteins-Structure Function and Genetics 17, 75-86.

Balbach, J., Forge, V., Van Nuland, N. A. J., Winder, S. L., Hore, P. J. \& Dobson, C. M. (1995). Following protein folding in real time using NMR spectroscopy. Nature Structural \& Molecular Biology 2, 865-870. 
BALDwin, A. J. (2014). An exact solution for R2,eff in CPMG experiments in the case of two site chemical exchange. Journal of Magnetic Resonance 244, 114-124.

Banci, L., Barbieri, L., Bertini, I., Luchinat, E., Secci, E., Zhao, Y. \& Aricescu, A. R. (2013). Atomic-resolution monitoring of protein maturation in live human cells by NMR. Nature Chemical Biology 9, 297-299.

BaShir, Q., SCANU, S. \& Ubink, M. (2011). Dynamics in electron transfer protein complexes. Febs Journal 278, 1391-1400.

Bashir, Q., Volkov, A. N., Ullmann, G. M. \& Ubiink, M. (2010). Visualization of the encounter ensemble of the transient electron transfer complex of cytochrome $\mathrm{c}$ and cytochrome c peroxidase. Journal of the American Chemical Society 132, 241-247.

BAX, A. (2003). Weak alignment offers new NMR opportunities to study protein structure and dynamics. Protein Science 12, 1-16.

BeACH, H., Cole, R., Gill, M. L. \& LoRia, J. P. (2005). Conservation of mus-ms enzyme motions in the apo- and substrate-mimicked state. Journal of the American Chemical Society 127, 9167-9176.

BennetT, W. S. \& Steitz, T. A. (1978). Glucose-Induced Conformational Change in Yeast Hexokinase. Proceedings of the National Academy of Sciences of the United States of America 75, 4848-4852.

Bernado, P., Bertoncini, C. W., Griesinger, C., Zweckstetter, M. \& Blackledge, M. (2005). Defining long-range order and local disorder in native alpha-synuclein using residual dipolar couplings. Journal of the American Chemical Society 127, 17968-17969.

Bernado, P. \& Blackledge, M. (2004). Local dynamic amplitudes on the protein backbone from dipolar couplings: toward the elucidation of slower motions in biomolecules. Journal of the American Chemical Society 126, 7760-7761.

Bertini, I., Luchinat, C. \& Piccioli, M. (2001). [15] - Paramagnetic probes in Metalloproteins. In Methods in Enzymology, vol. 339 (eds. V. D. Thomas, L. James \& S. Uli), pp. 314-340. Academic Press.

Bhabha, G., Ekiert, D. C., Jennewein, M., ZmaseK, C. M., Tuttle, L. M., Kroon, G., Dyson, H. J., GodziK, A., Wilson, I. A. \& Wright, P. E. (2013). Divergent evolution of protein conformational dynamics in dihydrofolate reductase. Nature Structural \& Molecular Biology 20, 1243-U1262.

Bhabha, G., Lee, J., Ekiert, D. C., Gam, J., Wilson, I. A., Dyson, H. J., Benkovic, S. J. \& Wright, P. E. (2011). A dynamic knockout reveals that conformational fluctuations influence the chemical step of enzyme catalysis. Science 332, 234-238.

Bingham, R. J., Findlay, J. B. C., Hsieh, S. Y., Kalverda, A. P., Kjeliberg, A., Perazzolo, C., Phillips, S. E. V., Seshadri, K., Trinh, C. H., Turnbull, W. B., Bodenhausen, G. \& Homans, S. W. (2004). Thermodynamics of binding of 2-methoxy-3-isopropylpyrazine and 2-methoxy-3-isobutylpyrazine to the major urinary protein. Journal of the American Chemical Society 126, 1675-1681.

Binolfi, A., Fernandez, C. O., Sica, M. P., Delfino, J. M. \& Santos, J. (2012). Recognition between a short unstructured peptide and a partially folded fragment leads to the thioredoxin fold sharing native-like dynamics. Proteins-Structure Function and Bioinformatics 80, $1448-1464$.

Birtalan, S. C., Phillips, R. M. \& Ghosh, P. (2002). Three-dimensional secretion signals in chaperone-effector complexes of bacterial pathogens. Molecular Cell 9, 971-980.

BLосн, F. (1946). Nuclear induction. Physical Review 70, 460-474.

Bloembergen, N. \& Morgan, L. O. (1961). Proton relaxation times in paramagnetic solutions. Effects of electron Spin relaxation. The Journal of Chemical Physics 34, 842-850.

Boehr, D. D., Dyson, H. J. \& Wright, P. E. (2006a). An NMR perspective on enzyme dynamics. Chemical Reviews 106, 3055-3079.

Boehr, D. D., Mcelheny, D., Dyson, H. J. \& Wright, P. E. (2006b). The dynamic energy landscape of dihydrofolate reductase catalysis. Science 313, 1638-1642.

Boenr, D. D., Nussinov, R. \& Wright, P. E. (2009). The role of dynamic conformational ensembles in biomolecular recognition (vol 5, pg 789, 2009). Nature Chemical Biology 5, 954-954.

Boos, W. \& Shuman, H. (1998). Maltose/maltodextrin system of Escherichia coli: transport, metabolism, and regulation. Microbiology and Molecular Biology Reviews 62, 204-229.

Bosshard, H. R. (2001). Molecular recognition by induced fit: how fit is the concept? News in Physiological Sciences 16, 171-173.

Bouvignies, G., Bernado, P., Meier, S., Cho, K., Grzesiek, S., Bruschweiler, R. \& Blackledge, M. (2005). Identification of slow correlated motions in proteins using residual dipolar and hydrogen-bond scalar couplings. Proceedings of the National Academy of Sciences of the United States of America 102, 13885-13890.

Bouvignies, G. \& KAY, L. E. (2012a). A 2D C-13-CEST experiment for studying slowly exchanging protein systems using methyl probes: an application to protein folding. Journal of Biomolecular NMR 53, 303-310.

Bouvignies, G. \& KAY, L. E. (2012b). Measurement of proton chemical shifts in invisible states of slowly exchanging protein systems by chemical exchange saturation transfer. Journal of Physical Chemistry B 116, 14311-14317.

Bouvignies, G., Markwick, P., Bruscheweiler, R. \& Blackledge, M. (2006). Simultaneous determination of protein backbone structure and dynamics from residual dipolar couplings. Journal of the American Chemical Society 128, 15100-15101.

Bouvignies, G., Markwick, P. R. L. \& Blackiedge, M. (2007). Simultaneous definition of high resolution protein structure and backbone conformational dynamics using NMR residual dipolar couplings. Chemphyschem: A European Journal of Chemical Physics and Physical Chemistry 8, 1901-1909.

Bouvignies, G., Vallurupalli, P., Hansen, D. F., Correia, B. E., Lange, O., Bah, A., Vernon, R. M., Dahlquist, F. W., Baker, D. \& Kay, L. E. (2011). Solution structure of a minor and transiently formed state of a T4 lysozyme mutant. Nature 477, 111-U134.

Bracken, C., Carr, P. A., Cavanagh, J. \& Palmer, A. G. (1999). Temperature dependence of intramolecular dynamics of the basic leucine zipper of GCN4: implications for the entropy of association with DNA. Journal of Molecular Biology 285, 2133-2146.

Braig, K., Otwinowski, Z., Hegde, R., Boisvert, D. C., Joachimiak, A., Horwich, A. L. \& Sigler, P. B. (1994). The crystal structure of the bacterial chaperonln GroEL at 2.8 A. Nature 371, 578-586. 
Briggman, K. B. \& Tolman, J. R. (2003). De Novo determination of bond orientations and order parameters from residual dipolar couplings with high accuracy. Journal of the American Chemical Society 125, 10164-10165.

BRUBAKER, R. R. (1991). Factors promoting acute and chronic diseases caused by Yersiniae. Clinical Microbiology Reviews 4, $309-324$.

Bryn Fenwick, R., Orellana, L., Esteban-Martin, S., Orozco, M. \& Salvatella, X. (2014). Correlated motions are a fundamental property of beta-sheets. Nature Communications 5, Article number 4070.

Buckie, A. M., Schreiber, G. \& Fersht, A. R. (1994). Protein-protein recognition - crystal structural-analysis of a Barnase Barstar complex at 2.0-angstrom resolution. Biochemistry 33, 8878-8889.

Buckle, A. M., Zahn, R. \& Fersht, A. R. (1997). A structural model for GroEL-polypeptide recognition. Proceedings of the National Academy of Sciences of the United States of America 94, 3571-3575.

BUKAU, B. \& WALKER, G. C. (1989). Cellular defects caused by deletion of the Escherichia coli dnaK gene indicate roles for heat shock protein in normal metabolism. Journal of Bacteriology 171, 2337-2346.

Bukau, B., Weissman, J. \& Horwich, A. (2006). Molecular chaperones and protein quality control. Cell 125, 443-451.

Butterwick, J. A. \& Palmer, A. G. (2006). An inserted Gly residue fine tunes dynamics between mesophilic and thermophilic ribonucleases $\mathrm{H}$. Protein Science 15, 2697-2707.

Camilloni, C., Cavalli, A. \& Vendruscolo, M. (2013). Assessment of the use of NMR chemical shifts as replica-averaged structural restraints in molecular dynamics simulations to characterize the dynamics of proteins. The Journal of Physical Chemistry B 117, 1838-1843.

Camilloni, C., Robustelli, P., Simone, A. D., Cavalli, A. \& Vendruscolo, M. (2012). Characterization of the conformational equilibrium between the two major substates of RNase A using NMR chemical shifts. Journal of the American Chemical Society 134, $3968-3971$.

Camilloni, C., Sahakyan, A. B., Holliday, M. J., Isern, N. G., Zhang, F. L., Eisenmesser, E. Z. \& Vendruscolo, M. (2014). Cyclophilin A catalyzes proline isomerization by an electrostatic handle mechanism. Proceedings of the National Academy of Sciences of the United States of America 111, 10203-10208.

Cammarata, M., Levantino, M., Schotte, F., Anfinrud, P. A., Ewald, F., Choi, J., Cupane, A., Wulff, M. \& Ihee, H. (2008). Tracking the structural dynamics of proteins in solution using time-resolved wide-angle X-ray scattering. Nature Methods 5, 881-886.

CAO, S., FÜrTig, B., SCHWAlbe, H. \& ChEN, S.-J. (2010). Folding kinetics for the conformational switch between alternative RNA structures. The Journal of Physical Chemistry B 114, 13609-13615.

Carr, H. Y. \& Purcell, E. M. (1954). Effects of diffusion on free precession in nuclear magnetic resonance experiments. Physical Review 94 , 630-638.

CARVER, J.P. \& RichardS, R.E. (1972). A general two-site solution for the chemical exchange produced dependence of T2 upon the Carr-Purcell pulse separation. Journal of Magnetic Resonance 6, 89-105.

Cavalli, A., Salvatella, X., Dobson, C. M. \& Vendruscolo, M. (2007). Protein structure determination from NMR chemical shifts. Proceedings of the National Academy of Sciences of the United States of America 104, 9615-9620.

Chang, S. L. \& TJANDRA, N. (2005). Temperature dependence of protein backbone motion from carbonyl C-13 and amide N-15 NMR relaxation. Journal of Magnetic Resonance 174, 43-53.

Chen, L. L. \& SigleR, P. B. (1999). The crystal structure of a GroEL/peptide complex: plasticity as a basis for substrate diversity. Cell $\mathbf{9 9}$, $757-768$.

Chevelkov, V., Xue, Y., Krishna Rao, D., Forman-Kay, J. \& Skrynnikov, N. (2010). 15NH/D-SOLEXSY experiment for accurate measurement of amide solvent exchange rates: application to denatured drkN SH3. Journal of Biomolecular NMR 46, 227-244.

Choudhury, D., Thompson, A., Stojanoff, V., Langermann, S., Pinkner, J., Hultgren, S. J. \& Knight, S. D. (1999). X-ray structure of the FimC-FimH chaperone-adhesin complex from uropathogenic Escherichia coli. Science 285, 1061-1066.

Clarke, J., Hounslow, A. M., Bycroft, M. \& Fersht, A. R. (1993). Local breathing and global unfolding in hydrogen exchange of barnase and its relationship to protein folding pathways. Proceedings of the National Academy of Sciences of the United States of America 90, 9837-9841.

Clore, G. M. (2008). Visualizing lowly-populated regions of the free energy landscape of macromolecular complexes by paramagnetic relaxation enhancement. Molecular Biosystems 4, 1058-1069.

Clore, G. M. \& Bewley, C. A. (2002). Using conjoined rigid body/torsion angle simulated annealing to determine the relative orientation of covalently linked protein domains from dipolar couplings. Journal of Magnetic Resonance 154, 329-335.

Clore, G. M. \& Gronenborn, A. M. (1998). New methods of structure refinement for macromolecular structure determination by NMR. Proceedings of the National Academy of Sciences of the United States of America 95, 5891-5898.

Clore, G. M., Gronenborn, A. M. \& BaX, A. (1998). A robust method for determining the magnitude of the fully asymmetric alignment tensor of oriented macromolecules in the absence of structural information. Journal of Magnetic Resonance 133, 216-221.

Clore, G. M. \& Iwahara, J. (2009). Theory, practice, and applications of paramagnetic relaxation enhancement for the characterization of transient low-population states of biological macromolecules and their complexes. Chemical Reviews 109, 4108-4139.

Clore, G. M. \& SChwieters, C. D. (2004a). Amplitudes of protein backbone dynamics and correlated motions in a small alpha/beta protein: correspondence of dipolar coupling and heteronuclear relaxation measurements. Biochemistry 43, 10678-10691.

Clore, G. M. \& SchwieTERs, C. D. (2004b). How much backbone motion in ubiquitin is required to account for dipolar coupling data measured in multiple alignment media as assessed by independent cross-validation? Journal of the American Chemical Society 126, $2923-2938$.

CLORE, G. M. \& SCHWIETERS, C. D. (2006). Concordance of residual dipolar couplings, backbone order parameters and crystallographic B-factors for a small $\alpha / \beta$ protein: a unified picture of high probability, fast atomic motions in proteins. Journal of Molecular Biology 355, 879-886.

Clore, G. M., Szabo, A., BaX, A., Kay, L. E., Driscoll, P. C. \& Gronenborn, A. M. (1990). Deviations from the simple 2-parameter model-free approach to the interpretation of N-15 nuclear magnetic-relaxation of proteins. Journal of the American Chemical Society 112, 4989-4991. 
Colvin, M. T., Silvers, R., Frohm, B., Su, Y., Linse, S. \& Griffin, R. G. (2015). High resolution structural characterization of A $\beta 42$ amyloid fibrils by magic angle spinning NMR. Journal of the American Chemical Society 137, 7509-7518.

Cooper, A. \& Dryden, D.T.F. (1984). Allostery without conformational change - a plausible model. European Biophysics Journal with Biophysics Letters 11, 103-109.

Cooper, A., Johnson, C. M., Lakey, J. H. \& Nollmann, M. (2001). Heat does not come in different colours: entropy-enthalpy compensation, free energy windows, quantum confinement, pressure perturbation calorimetry, solvation and the multiple causes of heat capacity effects in biomolecular interactions. Biophysical Chemistry 93, 215-230.

Cordier, F., Hartmann, B., Rogowski, M., Affolter, M. \& Grzesiek, S. (2006). DNA recognition by the Brinker repressor - An extreme case of coupling between binding and folding. Journal of Molecular Biology 361, 659-672.

Csermely, P., Palotai, R. \& Nussinov, R. (2010). Induced fit, conformational selection and independent dynamic segments: an extended view of binding events. Trends in Biochemical Sciences 35, 539-546.

Danielsson, J., Inomata, K., Murayama, S., Tochio, H., Lang, L., Shirakawa, M. \& Oliveberg, M. (2013). Pruning the ALS-associated protein SOD1 for in-cell NMR. Journal of the American Chemical Society 135, 10266-10269.

Danielsson, J., Mu, X., Lang, L., Wang, H., Binolfi, A., Theillet, F.-X., Bekei, B., Logan, D. T., Selenko, P., Wennerström, H. \& Oliveberg, M. (2015). Thermodynamics of protein destabilization in live cells. Proceedings of the National Academy of Sciences of the United States of America 112, 12402-12407.

Davis, D. G., Perlman, M. E. \& London, R. E. (1994). Direct measurements of the dissociation-rate constant for inhibitor-Enzyme complexes via the T1 $\rho$ and T2 (CPMG) methods. Journal of Magnetic Resonance, Series B 104, 266-275.

Davulcu, O., Flynn, P. F., Chapman, M. S. \& Skalicky, J. J. (2009). Intrinsic domain and loop dynamics commensurate with catalytic turnover in an induced-fit enzyme. Structure 17, 1356-1367.

Dellus-Gur, E., Elias, M., Caselli, E., Prati, F., Salverda, M. L. M., De Visser, J. A. G. M., Fraser, J. S. \& Tawfik, D. S. (2015). Negative epistasis and evolvability in TEM-1 $\beta$-Lactamase- the thin line between an Enzyme's conformational freedom and disorder. Journal of Molecular Biology 427, 2396-2409.

Demers, J. P. \& Mittermaier, A. (2009). Binding mechanism of an SH3 domain studied by NMR and ITC. Journal of the American Chemical Society 131, 4355-4367.

Deupi, X. \& Kobilka, B. K. (2010). Energy landscapes as a tool to integrate GPCR structure, dynamics, and function. Physiology 25, $293-303$.

Diehl, C., Engstrom, O., Delaine, T., Hakansson, M., Genheden, S., Modig, K., Leffler, H., Ryde, U., Nilsson, U. J. \& AkKe, M. (2010). Protein flexibility and conformational entropy in ligand design targeting the carbohydrate recognition domain of galectin-3. Journal of the American Chemical Society 132, 14577-14589.

Diehl, C., Genheden, S., Modig, K., Ryde, U. \& AkKe, M. (2009). Conformational entropy changes upon lactose binding to the carbohydrate recognition domain of galectin-3. Journal of Biomolecular NMR 45, 157-169.

Doucet, N., Khirich, G., Kovrigin, E. L. \& Loria, J. P. (2011). Alteration of hydrogen bonding in the vicinity of histidine 48 disrupts millisecond motions in RNase A. Biochemistry 50, 1723-1730.

DunitZ, J. D. (1995). Win some, lose some - enthalpy-entropy compensation in weak intermolecular interactions. Chemistry \& Biology 2, 709-712.

Dyson, H. J. \& Wright, P. E. (2004). Unfolded proteins and protein folding studied by NMR. Chemical Reviews 104, 3607-3622.

Dyson, H. J. \& WRight, P. E. (2005). Intrinsically unstructured proteins and their functions. Nature Reviews Molecular Cell Biology 6, 197-208.

Eisenmesser, E. Z., Bosco, D. A., AKKe, M. \& Kern, D. (2002). Enzyme dynamics during catalysis. Science 295, 1520-1523.

Eisenmesser, E. Z., Millet, O., Labeikovsky, W., Korzhnev, D. M., Wolf-Watz, M., Bosco, D. A., Skalicky, J. J., Kay, L. E. \& Kern, D. (2005). Intrinsic dynamics of an enzyme underlies catalysis. Nature 438, 117-121.

ELLIS, R. J. (1996). Discovery of molecular chaperones. Cell Stress \& Chaperones 1, 155-160.

ELLIS, R. J. (2003). Protein folding: importance of the Anfinsen cage. Current Biology 13, R881-R883.

Englander, S. W. \& Kallenbach, N. R. (1983). Hydrogen-exchange and structural dynamics of proteins and nucleic-acids. Quarterly Reviews of Biophysics, 16, 521-655.

Esteban-Martin, S., Fenwick, R. B., Aden, J., Cossins, B., Bertoncini, C. W., Guallar, V., Wolf-Watz, M. \& Salvatella, X. (2014). Correlated inter-domain motions in Adenylate Kinase. PLoS Computational Biology 10, e1003721.

Esteban-Martin, S., Fenwick, R. B. \& Salvatella, X. (2010). Refinement of ensembles describing unstructured proteins using NMR residual dipolar couplings. Journal of the American Chemical Society 132, 4626-4632.

Evenäs, J., Tugarinov, V., Skrynnikov, N. R., Goto, N. K., Muhandiram, R. \& Kay, L. E. (2001). Ligand-induced structural changes to maltodextrin-binding protein as studied by solution NMR spectroscopy. Journal of Molecular Biology 309, 961-974.

Farber, P. J. \& Mittermaier, A. (2011). Concerted dynamics link allosteric sites in the PBX Homeodomain. Journal of Molecular Biology 405, 819-830.

Farber, P. J., Slager, J. \& Mittermaier, A. K. (2012). Local folding and misfolding in the PBX homeodomain from a three-state analysis of CPMG relaxation dispersion NMR data. The Journal of Physical Chemistry. B 116, 10317-10329.

Farrow, N., Zhang, O., Forman-KaY, J. \& Kay, L. (1994a). A heteronuclear correlation experiment for simultaneous determination of 15N longitudinal decay and chemical exchange rates of systems in slow equilibrium. Journal of Biomolecular NMR 4, 727-734.

Farrow, N. A., Muhandiram, R., Singer, A. U., Pascal, S. M., Kay, C. M., Gish, G., Shollson, S. E., Pawson, T., Forman-Kay, J. D. \& Kay, L. E. (1994b). Backbone dynamics of a free and a phosphopeptide-complexed Src homology-2 domain studied by N-15 NMR relaxation. Biochemistry 33, 5984-6003. 
Fawzi, N. L., Libich, D. S., Ying, J. F., Tugarinov, V. \& Clore, G. M. (2014). Characterizing methyl-bearing side chain contacts and dynamics mediating amyloid beta Protofibril interactions using C-13(methyl)-DEST and lifetime line broadening. Angewandte Chemie-International Edition 53, 10345-10349.

Fawzi, N. L., Ying, J. F., Ghirlando, R., Torchia, D. A. \& Clore, G. M. (2011). Atomic-resolution dynamics on the surface of amyloid-beta protofibrils probed by solution NMR. Nature 480, 268-U161.

FawZI, N. L., YING, J. F., Torchia, D. A. \& Clore, G. M. (2010). Kinetics of Amyloid beta monomer-to-oligomer exchange by NMR relaxation. Journal of the American Chemical Society 132, 9948-9951.

Fawzi, N. L., Ying, J.F., Torchia, D. A. \& Clore, G. M. (2012). Probing exchange kinetics and atomic resolution dynamics in high-molecular-weight complexes using dark-state exchange saturation transfer NMR spectroscopy. Nature Protocols 7, $1523-1533$.

Fenwick, R. B., Esteban-Martin, S., Richter, B., Lee, D., Walter, K. F. A., Milovanovic, D., Becker, S., Lakomek, N. A., Griesinger, C. \& SALVATelLA, X. (2011). Weak long-range correlated motions in a surface patch of ubiquitin involved in molecular recognition. Journal of the American Chemical Society 133, 10336-10339.

Fenwick, R. B., Esteban-Martin, S. \& Salvatella, X. (2010). Influence of experimental uncertainties on the properties of ensembles derived from NMR residual dipolar couplings. Journal of Physical Chemistry Letters 1, 3438-3441.

Fenwick, R. B., Van Den Bedem, H., Fraser, J. S. \& Wright, P. E. (2014). Integrated description of protein dynamics from room-temperature X-ray crystallography and NMR. Proceedings of the National Academy of Sciences of the United States of America 111, E445-E454.

Ferreiro, D. U., Hegler, J. A., Komives, E. A. \& Wolynes, P. G. (2007). Localizing frustration in native proteins and protein assemblies. Proceedings of the National Academy of Sciences of the United States of America 104, 19819-19824.

Ferreiro, D. U., Komives, E. A. \& Wolynes, P. G. (2014). Frustration in biomolecules. Quarterly Reviews of Biophysics 47, $285-363$.

Fersht, A. (2000). Structure and Mechanism in Protein Science. New York: W.H. Freeman and Company.

Fiaux, J., Bertelsen, E. B., Horwich, A. L. \& Wuthrich, K. (2002). NMR analysis of a 900 K GroEL GroES complex. Nature 418, $207-211$.

Fierke, C. A., Johnson, K. A. \& Benkovic, S. J. (1987). Construction and evaluation of the kinetic scheme associated with dihydrofolate reductase from Escherichia coli. Biochemistry 26, 4085-4092.

FISCHER, E. (1894). The influence of configuration on enzyme activity (Translated from German). Deutsche Chemische Gesellschaft 27, 2984-2993.

Foote, J. \& Milstein, C. (1994). Conformational isomerism and the diversity of antibodies. Proceedings of the National Academy of Sciences of the United States of America 91, 10370-10374.

Forsen, S. \& Hoffman, R. A. (1963). Study of moderately rapid chemical exchange reactions by means of nuclear magnetic double resonance. Journal of Chemical Physics 39, 2892-\&.

Fraser, J. S., Clarkson, M. W., Degnan, S. C., Erion, R., Kern, D. \& Alber, T. (2009). Hidden alternative structures of proline isomerase essential for catalysis. Nature 462, 669-U149.

Frauenfelder, H., Chen, G., Berendzen, J., Fenimore, P. W., Jansson, H., Mcmahon, B. H., Stroe, I. R., Swenson, J. \& Young, R. D. (2009). A unified model of protein dynamics. Proceedings of the National Academy of Sciences of the United States of America 106, 5129-5134.

Frauenfelder, H., Sligar, S. G. \& Wolynes, P. G. (1991). The energy landscapes and motions of proteins. Science 254, 1598-1603.

Frederick, K. K., Marlow, M. S., Valentine, K. G. \& Wand, A. J. (2007). Conformational entropy in molecular recognition by proteins. Nature 448, 325-U323.

Frost, S., Ho, O., Login, F. H., Weise, C. F., Wolf-Watz, H. \& Wolf-Watz, M. (2012). Autoproteolysis and intramolecular dissociation of Yersinia YscU precedes secretion of its C-terminal polypeptide YscU(CC). Plos ONE 7, e49349.

Gagne, S. M., Tsuda, S., Spyracopoulos, L., Kay, L. E. \& Sykes, B. D. (1998). Backbone and methyl dynamics of the regulatory domain of troponin C: anisotropic rotational diffusion and contribution of conformational entropy to calcium affinity. Journal of Molecular Biology 278, $667-686$.

Gal, M., Schanda, P., Brutscher, B. \& Frydman, L. (2007). UltraSOFAST HMQC NMR and the repetitive acquisition of 2D protein spectra at $\mathrm{Hz}$ rates. Journal of the American Chemical Society 129, 1372-1377.

Gaponenko, V., Howarth, J. W., Columbus, L., Gasmi-Seabrook, G., Yuan, J., Hubbell, W. L. \& Rosevear, P. R. (2000). Protein global fold determination using site-directed spin and isotope labeling. Protein Science 9, 302-309.

Gardino, A. K., Villali, J., Kivenson, A., Lei, M., Liu, C. F., Steindel, P., Eisenmesser, E. Z., Labeikovsky, W., Wolf-Watz, M., Clarkson, M. W. \& Kern, D. (2009). Transient non-native hydrogen bonds promote activation of a signaling protein. Cell 139, 1109-1118.

Garrett, D. S., Seok, Y. J., Peterkofsky, A., Gronenborn, A. M. \& Clore, G. M. (1999). Solution structure of the 40,000 M-r phosphoryl transfer complex between the N-terminal domain of enzyme I and HPr. Nature Structural Biology 6, 166-173.

Gayathri, C., Bothner-By, A. A., VAn Zil, P. C. M. \& Maclean, C. (1982). Dipolar magnetic field effects in NMR spectra of liquids. Chemical Physics Letters 87, 192-196.

Gelis, I., Bonvin, A., Keramisanou, D., Koukaki, M., Gouridis, G., Karamanou, S., Economou, A. \& Kalodimos, C. G. (2007). Structural basis for signal-sequence recognition by the translocase motor SecA as determined by NMR. Cell 131, 756-769.

GemmeCKer, G., JAHNKe, W. \& KessleR, H. (1993). Measurement of fast proton-exchange rates in isotopically labeled compounds. Journal of the American Chemical Society 115, 11620-11621.

Gerlach, W. \& Stern, O. (1922). Der experimentelle Nachweis der Richtungsquantelung im Magnetfeld. Zeitschrift für Physik 9, 349-352. Gianni, S., Dogan, J. \& Jemth, P. (2014). Distinguishing induced fit from conformational selection. Biophysical Chemistry 189, 33-39.

Gillespie, J.R. \& Shortle, D. (1997a). Characterization of long-range structure in the denatured state of staphylococcal nuclease .1 . Paramagnetic relaxation enhancement by nitroxide spin labels. Journal of Molecular Biology 268, 158-169. 
Gillespie, J. R. \& Shortle, D. (1997b). Characterization of long-range structure in the denatured state of staphylococcal nuclease. II. distance restraints from paramagnetic relaxation and calculation of an ensemble of structures1. Journal of Molecular Biology 268, 170-184.

Grey, M. J., Wang, C. \& Palmer, A. G. (2003). Disulfide bond isomerization in basic pancreatic trypsin inhibitor: multisite chemical exchange quantified by CPMG relaxation dispersion and chemical shift modeling. Journal of the American Chemical Society 125, 14324-14335.

Gross, G., Gallopin, M., Vandame, M., Couprie, J., Stura, E., Zinn-Justin, S. \& Drevet, P. (2010). Conformational exchange is critical for the productivity of an oxidative folding intermediate with buried free Cysteines. Journal of Molecular Biology 403, $299-312$.

Gsponer, J., Christodoulou, J., Cavalli, A., Bui, J. M., Richter, B., Dobson, C. M. \& Vendruscolo, M. (2008). A coupled equilibrium shift mechanism in calmodulin-mediated signal transduction. Structure 16, 736-746.

Gutowsky, H. S., McCall, D. W. \& Suichter, C. P. (1953). Nuclear magnetic resonance multiplets in liquids. Journal of Chemical Physics 21 , 279-292.

Hammes, G. G., Chang, Y.C. \& OAs, T. G. (2009). Conformational selection or induced fit: a flux description of reaction mechanism. Proceedings of the National Academy of Sciences of the United States of America 106, 13737-13741.

Hansen, D. F., FenG, H. Q., Zhou, Z., BaI, Y. W. \& KaY, L. E. (2009). Selective characterization of microsecond motions in proteins by NMR relaxation. Journal of the American Chemical Society 131, 16257-16265.

Hansen, D. F., Vallurupalli, P., Lundström, P., Neudecker, P. \& Kay, L. E. (2008). Probing chemical shifts of invisible states of proteins with relaxation dispersion NMR spectroscopy: how well can we do? Journal of the American Chemical Society 130, 2667-2675.

Hansen, D. F., Yang, D. W., Feng, H. Q., Zhou, Z., Wiesner, S., BaI, Y. W. \& KaY, L. E. (2007). An exchange-free measure of N-15 transverse relaxation: an NMR spectroscopy application to the study of a folding intermediate with pervasive chemical exchange. Journal of the American Chemical Society 129, 11468-11479.

Hansen, M. R., Mueller, L. \& Pardi, A. (1998). Tunable alignment of macromolecules by filamentous phage yields dipolar coupling interactions. Nature Structural Biology 5, 1065-1074.

Hanson, J. A., Duderstadt, K., Watkins, L. P., Bhattacharyya, S., Brokaw, J., Chu, J. W. \& Yang, H. (2007). Illuminating the mechanistic roles of enzyme conformational dynamics. Proceedings of the National Academy of Sciences of the United States of America 104, 18055-18060.

Härd, T., Kellenbach, E., Boelens, R., Maler, B. A., Dahlman, K., Freedman, L. P., Carlstedtduke, J., Yamamoto, K. R., Gustafsson, J. A. \& Kaptein, R. (1990). Solution structure of the glucocorticoid receptor DNA-binding domain. Science 249, 157-160.

Harman, J. G. (2001). Allosteric regulation of the cAMP receptor protein. Biochimica Et Biophysica Acta-Protein Structure and Molecular Enzymology 1547, 1-17.

Harper, J. W. \& Schulman, B. A. (2006). Structural complexity in ubiquitin recognition. Cell 124, 1133-1116.

Harper, S. M., Neil, L. C., Day, I. J., Hore, P. J. \& Gardner, K. H. (2004). Conformational Changes in a Photosensory LOV Domain Monitored by Time-Resolved NMR Spectroscopy. Journal of the American Chemical Society 126, 3390-3391.

Hastings, C. A., Lee, S. Y., Cho, H.S., Yan, D., Kustu, S. \& Wemmer, D.E. (2003). High-resolution solution structure of the beryllofluoride-activated NtrC receiver domain. Biochemistry 42, 9081-9090.

Haupt, C., Patzschke, R., Weininger, U., Gröger, S., Kovermann, M. \& Balbach, J. (2011). Transient enzyme-substrate recognition monitored by real-time NMR. Journal of the American Chemical Society 133, 11154-11162.

Henzler-Wildman, K. A., Thai, V., Lei, M., Ott, M., Wolf-Watz, M., Fenn, T., Pozharski, E., Wilson, M. A., Petsko, G. A., Karplus, M., HÜBNER, C. G. \& KeRN, D. (2007). Intrinsic motions along an enzymatic reaction trajectory. Nature 450, 838-844.

Hicke, L., SChUbert, H. L. \& Hill, C. P. (2005). Ubiquitin-binding domains. Nature Reviews Molecular Cell Biology 6, 610-621.

Hoeltzli, S. D. \& Frieden, C. (1995). Stopped-flow NMR spectroscopy: real-time unfolding studies of 6-19F-tryptophan-labeled Escherichia coli dihydrofolate reductase. Proceedings of the National Academy of Sciences of the United States of America 92, 9318-9322.

Holliday, M. J., Camilloni, C., Armstrong, G. S., Isern, N. G., Zhang, F., Vendruscolo, M. \& Eisenmesser, E. Z. (2015). Structure and dynamics of GeoCyp: a Thermophilic Cyclophilin with a novel substrate binding mechanism that functions efficiently at low temperatures. Biochemistry 54, 3207-3217.

Homans, S. W. (2005). Probing the binding entropy of ligand-protein interactions by NMR. Chembiochem 6, 1585-+.

Horst, R., Bertelsen, E. B., FiauX, J., Wider, G., Horwich, A. L. \& Wüthrich, K. (2005). Direct NMR observation of a substrate protein bound to the chaperonin GroEL. Proceedings of the National Academy of Sciences of the United States of America 102, 12748-12753.

Horst, R., Fenton, W. A., Englander, S. W., Wüthrich, K. \& Horwich, A. L. (2007). Folding trajectories of human dihydrofolate reductase inside the GroEL-GroES chaperonin cavity and free in solution. Proceedings of the National Academy of Sciences of the United States of America 104, 20788-20792.

Horwich, A. L. \& Fenton, W. A. (2009). Chaperonin-mediated protein folding: using a central cavity to kinetically assist polypeptide chain folding. Quarterly Reviews of Biophysics 42, 83-116.

Hubbell, W. L., Cafiso, D. S. \& Altenbach, C. (2000). Identifying conformational changes with site-directed spin labeling. Nature Structural Biology 7, 735-739.

Hwang, H., Pierce, B., Mintseris, J., Janin, J. \& Weng, Z. P. (2008). Protein-protein docking benchmark version 3.0. Proteins-Structure Function and Bioinformatics 73, 705-709.

Hwang, T. L., VAn ZiJl, P. C. \& MorI, S. (1998). Accurate quantitation of water-amide proton exchange rates using the phase-modulated CLEAN chemical EXchange (CLEANEX-PM) approach with a Fast-HSQC (FHSQC) detection scheme. Journal of biomolecular NMR 11, 221-226.

HvidT, A. \& Nielsen, S. O. (1966). Hydrogen exchange in proteins. Advances in Protein Chemistry 21, 287-386. 
Hyeon, C. \& OnUChic, J. N. (2007). Mechanical control of the directional stepping dynamics of the kinesin motor. Proceedings of the National Academy of Sciences of the United States of America 104, 17382-17387.

Ikura, M., Clore, G. M., Gronenborn, A. M., Zhu, G., Klee, C. B. \& BaX, A. (1992). Solution structure of a calmodulin-target peptide complex by multidimensional NMR. Science 256, 632-638.

Ishima, R., Freedberg, D. I., Wang, Y. X., Louis, J. M. \& Torchia, D. A. (1999). Flap opening and dimer-interface flexibility in the free and inhibitor-bound HIV protease, and their implications for function. Structure Fold Des 7, 1047-1055.

Ishima, R. \& Torchia, D. (1999). Estimating the time scale of chemical exchange of proteins from measurements of transverse relaxation rates in solution. Journal of Biomolecular NMR 14, 369-372.

IsHima, R. \& TorchiA, D. A. (2000). Protein dynamics from NMR. Nature Structural Biology 7, 740-743.

Ishima, R. \& Torchia, D. A. (2003). Extending the range of amide proton relaxation dispersion experiments in proteins using a constant-time relaxation-compensated CPMG approach. Journal of Biomolecular NMR 25, 243-248.

Ishima, R. \& Torchia, D. A. (2005). Error estimation and global fitting in transverse-relaxation dispersion experiments to determine chemical-exchange parameters. Journal of Biomolecular NMR 32, 41-54.

Iwahara, J. \& Clore, G. M. (2006). Detecting transient intermediates in macromolecular binding by paramagnetic NMR. Nature 440, $1227-1230$.

IwAhara, J., Schwieters, C. D. \& Clore, G. M. (2004). Ensemble approach for NMR structure refinement against H-1 paramagnetic relaxation enhancement data arising from a flexible paramagnetic group attached to a macromolecule. Journal of the American Chemical Society 126, 5879-5896.

Janin, J., Bahadur, R. P. \& Chakrabarti, P. (2008). Protein-protein interaction and quaternary structure. Quarterly Reviews of Biophysics 41, $133-180$.

Jankowski, W., Saleh, T., Pai, M.-T., SRiram, G., Birge, R. B. \& Kalodimos, C. G. (2012). Domain organization differences explain Bcr-Abl's preference for CrkL over CrkII. Nature Chemical Biology 8, 590-596.

Jeener, J., Meier, B. H., Bachmann, P. \& ERnst, R. R. (1979). Investigation of exchange processes by 2-dimensional nmr-spectroscopy. Journal of Chemical Physics 71, 4546-4553.

Jeng, M. F., Englander, S. W., Pardue, K., Rogalskyj, J. S. \& Mclendon, G. (1994). Structural dynamics in an electron-transfer complex. Nature Structural Biology 1, 234-238.

Jenik, M., Gonzalo Parra, R., Radusky, L. G., Turjanski, A., Wolynes, P. G. \& Ferreiro, D. U. (2012). Protein frustratometer: a tool to localize energetic frustration in protein molecules. Nucleic Acids Research 40, W348-W351.

Jensen, M. R., Houben, K., Lescop, E., Blanchard, L., Ruigrok, R. W. H. \& Blackledge, M. (2008). Quantitative conformational analysis of partially folded proteins from residual dipolar couplings: application to the molecular recognition element of Sendai virus nucleoprotein. Journal of the American Chemical Society 130, 8055-8061.

Jensen, M. R., RuigroK, R. W. H. \& Blackledge, M. (2013). Describing intrinsically disordered proteins at atomic resolution by NMR. Current Opinion in Structural Biology 23, 426-435.

Jensen, M. R., Zweckstetter, M., Huang, J.-R. \& Blackledge, M. (2014). Exploring free-energy landscapes of intrinsically disordered proteins at atomic resolution using NMR spectroscopy. Chemical Reviews 114, 6632-6660.

Jonker, H. R. A., Wechselberger, R. W., Boelens, R., Folkers, G. E. \& Kaptein, R. (2005). Structural properties of the promiscuous VP16 activation domain. Biochemistry 44, 827-839.

Kabiri, M. \& Unsworth, L. D. (2014). Application of isothermal titration calorimetry for characterizing thermodynamic parameters of biomolecular interactions: peptide self-assembly and protein adsorption case studies. Biomacromolecules 15, 3463-3473.

Katoh, H., Yoshinaga, M., Yanagita, T., Ohgi, K., Irie, M., Beintema, J. J. \& Meinsma, D. (1986). Kinetic-studies on turtle pancreatic ribonuclease - a comparative-study of the base specificities of the B2 and P0 sites of bovine pancreatic Ribonuclease-a and TURTLE PANCREATIC RIBONUCLEASE. Biochimica et Biophysica acta 873, 367-371.

KAY, L. E. (1998). Protein dynamics from NMR. Nature Structural Biology 5(Suppl), 513-517.

KaY, L.E., TorchiA, D.A. \& BaX, A. (1989). Backbone dynamics of proteins as studied by N-15 inverse detected heteronuclear nmr-spectroscopy - application to staphylococcal nuclease. Biochemistry 28, 8972-8979.

Keane, S. C., Heng, X., Lu, K., Kharytonchy,, S., Ramakrishnan, V., Carter, G., Barton, S., Hosic, A., Florwick, A., Santos, J., Bolden, N. C., Mccowin, S., Case, D. A., Johnson, B. A., Salemi, M., Telesnitsky, A. \& Summers, M. F. (2015). Structure of the HIV-1 RNA packaging signal. Science 348, 917-921.

KemPF, J. G. \& LoriA, J. P. (2003). Protein dynamics from solution NMR - Theory and applications. Cell Biochemistry and Biophysics 37, 187211.

Keramisanou, D., Biris, N., Gelis, I., Sianidis, G., Karamanou, S., Economou, A. \& Kalodimos, C. G. (2006). Disorder-order folding transitions underlie catalysis in the helicase motor of SecA. Nature Structural \& Molecular Biology 13, 594-602.

Kern, D., Eisenmesser, E.Z. \& Wolf-Watz, M. (2005). Enzyme dynamics during catalysis measured by NMR spectroscopy. In Nuclear Magnetic Resonance of Biological Macromolecules, Part C, vol. 394 (ed. T. L. JAMES), pp. 507-524. San Diego, USA: Elsevier Academic Press Inc.

Kern, D., Volkman, B. F., Luginbuhl, P., Nohaile, M. J., Kustu, S. \& Wemmer, D. E. (1999). Structure of a transiently phosphorylated switch in bacterial signal transduction. Nature 402, 894-898.

Kerns, S. J., Agafonov, R. V., Cho, Y. J., Pontiggia, F., Otten, R., Pachov, D. V., Kutter, S., Phung, L. A., Murphy, P. N., Thai, V., Alber, T., HaGAN, M. F. \& KeRn, D. (2015). The energy landscape of adenylate kinase during catalysis. Nature Structural \& Molecular Biology 22, $124-131$. 
Kim, S., Kim, D., Jung, H. H., LeE, I. H., Kim, J. I., Suh, J. Y. \& Jon, S. (2012). Bio-inspired design and potential biomedical applications of a novel class of high-affinity peptides. Angewandte Chemie-International Edition 51, 1890-1894.

Kimsey, I. J., Petzold, K., Sathyamoorthy, B., Stein, Z. W. \& Al-Hashimi, H. M. (2015). Visualizing transient Watson-Crick-like mispairs in DNA and RNA duplexes. Nature 519, 315-+.

Kloiber, K., Spitzer, R., Grutsch, S., Kreutz, C. \& Tollinger, M. (2011). Longitudinal exchange: an alternative strategy towards quantification of dynamics parameters in ZZ exchange spectroscopy. Journal of Biomolecular NMR 51, 123-129.

Koide, S., JAhnKe, W. \& Wright, P. (1995). Measurement of intrinsic exchange rates of amide protons in a 15N-labeled peptide. Journal of Biomolecular NMR 6, 306-312.

Korzhnev, D. M., Religa, T. L., Banachewicz, W., Fersht, A. R. \& Kay, L. E. (2010). A transient and low-populated protein-folding intermediate at atomic resolution. Science 329, 1312-1316.

Korzhnev, D. M., Salvatella, X., Vendruscolo, M., Di Nardo, A. A., Davidson, A. R., Dobson, C. M. \& Kay, L. E. (2004). Low-populated folding intermediates of Fyn SH3 characterized by relaxation dispersion NMR. Nature 430, 586-590.

Kosen, P. A. (1989). Spin labeling of proteins. Methods in Enzymology 177, 86-121.

Koshland, D. E. (1958). Application of a theory of enzyme specificity to protein synthesis. Proceedings of the National Academy of Sciences of the United States of America 44, 98-104.

Koshland, D. E., Nemethy, G. \& Filmer, D. (1966). Comparison of experimental binding data and theoretical models in proteins containing subunits. Biochemistry 5, 365-385.

Kovermann, M., Aden, J., Grundstrom, C., Elisabeth Sauer-Eriksson, A., Sauer, U. H. \& Wolf-Watz, M. (2015). Structural basis for catalytically restrictive dynamics of a high-energy enzyme state. Nature Communications 6, Article number 7644.

Kovermann, M. \& Balbach, J. (2013). Dynamic control of the prolyl isomerase function of the dual-domain SlyD protein. Biophysical Chemistry 171, 16-23.

Kovermann, M., Zierold, R., Haupt, C., Low, C. \& Balbach, J. (2011). NMR relaxation unravels interdomain crosstalk of the two domain prolyl isomerase and chaperone SlyD. Biochimica Et Biophysica Acta-Proteins and Proteomics 1814, 873-881.

Kovrigin, E. L., Cole, R. \& Loria, J. P. (2003). Temperature dependence of the backbone dynamics of ribonuclease A in the ground state and bound to the inhibitor $5^{6}$-phosphothymidine $\left(3^{6}-5^{\circ}\right)$ pyrophosphate adenosine 3 '-phosphate. Biochemistry 42, 5279-5291.

Kovrigin, E. L., KempF, J. G., Grey, M. J. \& Loria, J. P. (2006). Faithful estimation of dynamics parameters from CPMG relaxation dispersion measurements. Journal of Magnetic Resonance 180, 93-104.

Kovrigin, E. L. \& LoriA, J. P. (2006). Enzyme dynamics along the reaction coordinate: critical role of a conserved residue. Biochemistry 45, 2636-2647.

Kumar, A. \& Balbach, J. (2015). Real-time protein NMR spectroscopy and investigation of assisted protein folding. Biochimica et Biophysica Acta (BBA) - General Subjects 1850, 1965-1972.

Lakomek, N. A., Fares, C., Becker, S., Carlomagno, T., Meiler, J. \& Griesinger, C. (2005). Side-chain orientation and hydrogen-bonding imprint supra-tau(c) motion on the protein backbone of ubiquitin. Angewandte Chemie-International Edition 44, 7776-7778.

Lakomek, N.-A., Walter, K. F. A., Fares, C., Lange, O. F., De Groot, B. L., Grubmueller, H., Brueschweiler, R., Munk, A., Becker, S., Meiler, J. \& GRIESINGER, C. (2008a). Self-consistent residual dipolar coupling based model-free analysis for the robust determination of nanosecond to microsecond protein dynamics. Journal of Biomolecular NMR 41, 139-155.

Lakomek, N. A., Lange, O. F., Walter, K. F. A., Fares, C., Egger, D., Lunkenheimer, P., Meiler, J., Grubmuller, H., Becker, S., De Groot, B. L. \& GrIesingeR, C. (2008b). Residual dipolar couplings as a tool to study molecular recognition of ubiquitin. Biochemical Society Transactions 36, 1433-1437.

LANDRY, S. J. \& Gierasch, L. M. (1991). The chaperonin groel binds a polypeptide in an alpha-helical conformation. Biochemistry 30, 73597362.

Lange, O. F., Lakomek, N. A., Fares, C., Schroder, G. F., Walter, K. F., Becker, S., Meiler, J., Grubmuller, H., Griesinger, C. \& De Groot, B. L. (2008). Recognition dynamics up to microseconds revealed from an RDC-derived ubiquitin ensemble in solution. Science 320, 1471-1475.

Latham, M.P., Zimmermann, G.R. \& PArdi, A. (2009). NMR chemical exchange as a probe for ligand-binding kinetics in a Theophylline-binding RNA aptamer. Journal of the American Chemical Society 131, 5052-5053.

Leder, L., Berger, C., Bornhauser, S., Wendt, H., Ackermann, F., Jelesarov, I. \& Bosshard, H. R. (1995). Spectroscopic, calorimetric, and kinetic demonstration of conformational adaptation in peptide antibody recognition. Biochemistry 34, 16509-16518.

Lee, S. Y., Pullen, L., Virgit, D. J., Castaneda, C. A., Abeykoon, D., Bolon, D. N. A. \& Fushman, D. (2014). Alanine scan of core positions in ubiquitin reveals links between dynamics, stability, and function. Journal of Molecular Biology 426, 1377-1389.

LEFfLER, J. E. (1955). The enthalpy-entropy relationship and its implications for organic chemistry. Journal of Organic Chemistry 20, $1202-1231$.

Lehtivarjo, J., Tuppurainen, K., Hassinen, T., Laatikainen, R. \& Peräkylä, M. (2012). Combining NMR ensembles and molecular dynamics simulations provides more realistic models of protein structures in solution and leads to better chemical shift prediction. Journal of Biomolecular NMR 52, 257-267.

Lendel, C., Bjerring, M., Dubnovitsky, A., Kelly, R. T., Filippov, A., Antzutkin, O. N., Nielsen, N. C. \& Härd, T. (2014). A hexameric peptide barrel as building block of amyloid- $\beta$ protofibrils. Angewandte Chemie International Edition 53, 12756-12760.

LEWANDowsKi, J. R. (2013). Advances in solid-state relaxation methodology for probing site-specific protein dynamics. Accounts of Chemical Research 46, 2018-2027.

Levy, R. M., Karplus, M. \& Wolynes, P. G. (1981). NMR relaxation parameters in molecules with internal motion: exact Langevin trajectory results compared with simplified relaxation models. Journal of the American Chemical Society 103, 5998-6011. 
LI, D. W. \& BRuschweILeR, R. (2009). A dictionary for protein side-chain entropies from NMR order parameters. Journal of the American Chemical Society 131, 7226-7227.

LI, D.-W. \& BRÜschweIleR, R. (2011). Iterative optimization of molecular mechanics force fields from NMR data of full-length proteins. Journal of Chemical Theory and Computation 7, 1773-1782.

Li, P. L., Martins, I. R. S., Amarasinghe, G. K. \& Rosen, M. K. (2008). Internal dynamics control activation and activity of the autoinhibited Vav DH domain. Nature Structural \& Molecular Biology 15, 613-618.

LI, Y. \& PALMER, A. III (2009). TROSY-selected ZZ-exchange experiment for characterizing slow chemical exchange in large proteins. Journal of Biomolecular NMR 45, 357-360.

Libich, D. S., FawZi, N. L., YING, J. F. \& Clore, G. M. (2013). Probing the transient dark state of substrate binding to GroEL by relaxation-based solution NMR. Proceedings of the National Academy of Sciences of the United States of America 110, 11361-11366.

Lin, X. C., Eddy, N. R., Noel, J. K., Whitford, P. C., Wang, Q. H., MA, J. P. \& Onuchic, J. N. (2014). Order and disorder control the functional rearrangement of influenza hemagglutinin. Proceedings of the National Academy of Sciences of the United States of America 111, $12049-12054$.

Lindgren, A. E., Karlberg, T., Thorsell, A. G., Hesse, M., Spjut, S., Ekblad, T., Andersson, C. D., Pinto, A. F., Weigelt, J., Hottiger, M. O., Linusson, A., Elofsson, M. \& SChÜler, H. (2013). PARP inhibitor with selectivity toward ADP-Ribosyltransferase ARTD3/PARP3. ACS Chemical Biology 8, 1698-1703.

Lindley, P. F., Bajaj, M., Evans, R. W., Garratt, R. C., Hasnain, S. S., Jhoti, H., Kuser, P., Neu, M., Patel, K., Sarra, R., Strange, R. \& Walton, A. (1993). The mechanism of iron uptake by transferrins: the structure of an $18 \mathrm{kDa}$ NII-domain fragment from duck ovotransferrin at $2 \cdot 3$ Å resolution. "Acta Crystallographica. Section D, Biological Crystallography" 49(Pt 2), 292-304.

Lindorff-Larsen, K., Best, R. B., Depristo, M. A., Dobson, C. M. \& Vendruscolo, M. (2005). Simultaneous determination of protein structure and dynamics. Nature 433, 128-132.

Lindorff-Larsen, K., Piana, S., Dror, R. O. \& Shaw, D. E. (2011). How fast-folding proteins fold. Science 334, 517-520.

LIPARI, G. \& SZABO, A. (1982a). Model-free approach to the interpretation of nuclear magnetic-resonance relaxation in macromolecules .1. Theory and range of validity. Journal of the American Chemical Society 104, 4546-4559.

LIPARI, G. \& SZABO, A. (1982b). Model-free approach to the interpretation of nuclear magnetic-resonance relaxation in macromolecules .2. Analysis of experimental results. Journal of the American Chemical Society 104, 4559-4570.

LonG, D., Bouvignies, G. \& Kay, L. E. (2014). Measuring hydrogen exchange rates in invisible protein excited states. Proceedings of the National Academy of Sciences of the United States of America 111, 8820-8825.

Loria, J. P., Rance, M. \& PAlmer, A. G. (1999). A relaxation-compensated Carr-Purcell-Meiboom-Gill sequence for charactherizing chemical exchange by NMR spectroscopy. Journal of the American Chemical Society 121, 2331-2332.

Lu, J., Cistola, D. P. \& LI, E. (2006). Analysis of ligand binding and protein dynamics of human retinoid X receptor alpha ligand-binding domain by nuclear magnetic resonance†. Biochemistry 45, 1629-1639.

Lundström, P., Mulder, F. A. A. \& AKKe, M. (2005). Correlated dynamics of consecutive residues reveal transient and cooperative unfolding of secondary structure in proteins. Proceedings of the National Academy of Sciences of the United States of America 102, 16984-16989.

Luz, Z. \& Meiвoom, S. (1963). Nuclear magnetic resonance study of the protolysis of trimethylammonium ion in aqueous solution—order of the reaction with respect to solvent. The Journal of Chemical Physics 39, 366-370.

MACIEJEWSKI, M., TJANDRA, N. \& BarLow, P. N. (2011). Estimation of interdomain flexibility of N-terminus of factor H using residual dipolar couplings. Biochemistry 50, 8138-8149.

Mäler, L., Blankenship, J., Rance, M. \& Chazin, W. J. (2000). Site-site communication in the EF-hand Ca2+-binding protein calbindin D-9k. Nature Structural Biology 7, 245-250.

Mainz, A., Religa, T. L., Sprangers, R., Linser, R., Kay, L. E. \& Reif, B. (2013). NMR spectroscopy of soluble protein complexes at one megadalton and beyond. Angewandte Chemie-International Edition 52, 8746-8751.

Marchanka, A., Simon, B., Althoff-Ospelt, G. \& Carlomagno, T. (2015). RNA structure determination by solid-state NMR spectroscopy. Nature Communications 6, Article number 7024.

Markin, C. J., Saltibus, L. F., Kean, M. J., Mckay, R. T., Xiao, W. \& Spyracopoulos, L. (2010). Catalytic proficiency of ubiquitin conjugation enzymes: balancing pKa suppression, entropy, and electrostatics. Journal of the American Chemical Society 132, 17775-17786.

Markwick, P. R. L., Cervantes, C. F., Abel, B. L., Komives, E. A., Blackledge, M. \& Mccammon, J. A. (2010). Enhanced conformational space sampling improves the prediction of chemical shifts in proteins. Journal of the American Chemical Society 132, 1220-1221.

Marlow, M. S., Dogan, J., Frederick, K. K., Valentine, K. G. \& Wand, A. J. (2010). The role of conformational entropy in molecular recognition by calmodulin. Nature Chemical Biology 6, 352-358.

Masterson, L. R., Cheng, C., Yu, T., Tonelli, M., Kornev, A., Taylor, S. S. \& Veglia, G. (2010). Dynamics connect substrate recognition to catalysis in protein kinase A. Nature Chemical Biology 6, 821-828.

Masterson, L. R., Shi, L., Metcalfe, E., Gao, J., Taylor, S. S. \& Veglia, G. (2011a). Dynamically committed, uncommitted, and quenched states encoded in protein kinase A revealed by NMR spectroscopy. Proceedings of the National Academy of Sciences of the United States of America 108, 6969-6974.

Masterson, L. R., Yu, T., Shi, L., Wang, Y., Gustavsson, M., Mueller, M. M. \& Veglia, G. (2011b). cAMP-dependent protein kinase A selects the excited state of the membrane substrate phospholamban. Journal of Molecular Biology 412, 155-164.

McconnelL, H. M. (1958). Reaction rates by nuclear magnetic resonance. Journal of Chemical Physics 28, 430-431.

Mcelheny, D., Schnell, J. R., Lansing, J. C., Dyson, H. J. \& Wright, P. E. (2005). Defining the role of active-site loop fluctuations in dihydrofolate reductase catalysis. Proceedings of the National Academy of Sciences of the United States of America 102, 5032-5037. 
Mcelroy, C., Manfredo, A., Wendt, A., Gollnick, P. \& Foster, M. (2002). TROSY-NMR studies of the 91 kDa TRAP protein reveal allosteric control of a gene regulatory protein by ligand-altered flexibility. Journal of Molecular Biology 323, 463-473.

Mcelroy, C. A., Manfredo, A., Gollnick, P. \& Foster, M. P. (2006). Thermodynamics of tryptophan-mediated activation of the trp RNA-binding attenuation protein. Biochemistry 45, 7844-7853.

Мегвоом, S. \& Gill, D. (1958). Modified spin-echo method for measuring nuclear relaxation times. Review of Scientific Instruments 29, 688691.

Meiler, J., Prompers, J. J., Peti, W., Griesinger, C. \& Bruschweiler, R. (2001). Model-free approach to the dynamic interpretation of residual dipolar couplings in globular proteins. Journal of the American Chemical Society 123, 6098-6107.

MeInHOLD, D. W. \& WRight, P. E. (2011). Measurement of protein unfolding/refolding kinetics and structural characterization of hidden intermediates by NMR relaxation dispersion. Proceedings of the National Academy of Sciences of the United States of America 108, 9078-9083.

Mendoza, J. A., Rogers, E., Lorimer, G. H. \& Horowitz, P. M. (1991). Chaperonins facilitate the in vitro folding of monomeric mitochondrial rhodanese. Journal of Biological Chemistry 266, 13044-13049.

Mesecar, A. D., Stoddard, B. L. \& Koshland, D. E. Jr (1997). Orbital steering in the catalytic power of enzymes: small structural changes with large catalytic consequences. Science 277, 202-206.

Michaelis, L. \& Menten, M. L. (1913). Die kinetik der invertinwirkung. Biochemische Zeitschrift 49, 333-369.

Michielssens, S., De Groot, B. L. \& Grubmueller, H. (2015). Binding affinities controlled by shifting conformational equilibria: opportunities and limitations. Biophysical Journal 108, 2585-2590.

Michielssens, S., Peters, J. H., Ban, D., Pratihar, S., Seeliger, D., Sharma, M., Giller, K., Sabo, T. M., Becker, S., Lee, D., Griesinger, C. \& De Groот, B. L. (2014). A designed conformational shift to control protein binding specificity. Angewandte Chemie-International Edition 53, 10367-10371.

Miletti, T., Farber, P. \& Mittermaier, A. (2011). Active site dynamics in NADH oxidase from Thermus thermophilus studied by NMR spin relaxation. Journal of Biomolecular NMR 51, 71-82.

Miller, S. B. M., Mogk, A. \& BuKau, B. (2015). Spatially organized aggregation of Misfolded proteins as cellular stress defense strategy. Journal of Molecular Biology 427, 1564-1574.

Millet, O., Loria, J. P., Kroenke, C. D., Pons, M. \& Palmer, A. G. (2000). The static magnetic field dependence of chemical exchange line broadening defines the NMR chemical shift time scale. Journal of the American Chemical Society 122, 2867-2877.

Millet, O., Muhandiram, D. R., Skrynnikov, N.R. \& Kay, L.E. (2002). Deuterium spin probes of side-chain dynamics in proteins. 1. Measurement of five relaxation rates per deuteron in C-13-labeled and fractionally $\mathrm{H}$-2-enriched proteins in solution. Journal of the American Chemical Society 124, 6439-6448.

Miloushev, V. Z., Bahna, F., Ciatto, C., Ahlsen, G., Honig, B., Shapiro, L. \& Palmer Iil, A. G. (2008). Dynamic properties of a type II cadherin adhesive domain: implications for the mechanism of strand-swapping of classical Cadherins. Structure 16, 1195-1205.

Miyashita, O., Onuchic, J. N. \& Wolynes, P. G. (2003). Nonlinear elasticity, proteinquakes, and the energy landscapes of functional transitions in proteins. Proceedings of the National Academy of Sciences of the United States of America 100, 12570-12575.

Miyashita, O., Wolynes, P. G. \& Onuchic, J. N. (2005). Simple energy landscape model for the kinetics of functional transitions in proteins. Journal of Physical Chemistry B 109, 1959-1969.

Monod, J., Wyman, J. \& ChangeuX, J. P. (1965). On the nature of allosteric transitions: a plausible model. Journal of Molecular Biology 12, 88-118.

Montelione, G. T. \& WAGNER, G. (1989). 2D Chemical exchange NMR spectroscopy by proton-detected heteronuclear correlation. Journal of the American Chemical Society 111, 3096-3098.

Morgan, W. D., Birdsall, B., Nieto, P. M., Gargaro, A. R. \& Feeney, J. (1999). 1H/15N HSQC NMR studies of ligand carboxylate group interactions with arginine residues in complexes of brodimoprim analogues and Lactobacillus casei Dihydrofolate Reductase. Biochemistry $\mathbf{3 8}$ 2127-2134.

Morrison, E. A., Dekoster, G. T., Dutta, S., Vafabakhsh, R., Clarkson, M. W., Bahl, A., Kern, D., Ha, T. \& Henzler-Wildman, K. A. (2012). Antiparallel EmrE exports drugs by exchanging between asymmetric structures. Nature 481, 45-50.

Mulder, F. A., Mittermaier, A., Hon, B., Dahlquist, F. W. \& Kay, L. E. (2001a). Studying excited states of proteins by NMR spectroscopy. Nature Structural Biology 8, 932-935.

Mulder, F. A., Skrynnikov, N. R., Hon, B., Dahlquist, F. W. \& Kay, L. E. (2001b). Measurement of slow (micros-ms) time scale dynamics in protein side chains by (15)N relaxation dispersion NMR spectroscopy: application to Asn and Gln residues in a cavity mutant of T4 lysozyme. Journal of the American Chemical Society 123, 967-975.

Müller, C. W., Schlauderer, G. J., Reinstein, J. \& Schulz, G. E. (1996). Adenylate kinase motions during catalysis: an energetic counterweight balancing substrate binding. Structure 4, 147-156.

Müller, C. W. \& Schulz, G. E. (1992). Structure of the complex between adenylate kinase from Escherichia-coli and the inhibitor AP5A refined at 1.9 a resolution - a model for a catalytic transition-state. Journal of Molecular Biology 224, 159-177.

Nagarajan, S., Amir, D., Grupi, A., Goldenberg, D. P., Minton, A. P. \& HaAs, E. (2011). Modulation of functionally significant conformational Equilibria in Adenylate kinase by high concentrations of trimethylamine oxide attributed to volume exclusion. Biophysical Journal 100, 2991-2999.

Nagata, T., Gupta, V., Sorce, D., Kim, W. Y., Sali, A., Chait, B. T., Shigesada, K., Ito, Y. \& Werner, M. H. (1999). Immunoglobulin motif DNA recognition and heterodimerization of the PEBP2/CBF Runt domain. Nature Structural Biology 6, 615-619.

Nikolova, E. N., Kim, E., Wise, A. A., O’BRIEN, P. J., Andricioaei, I. \& Al-Hashimi, H. M. (2011). Transient Hoogsteen base pairs in canonical duplex DNA. Nature 470, 498-U484. 
Nirmala, N. R. \& WAGNER, G. (1988). Measurement of 13C relaxation times in proteins by two-dimensional heteronuclear 1H-13C correlation spectroscopy. Journal of the American Chemical Society 110, 7557-7558.

Nirmala, N. R. \& WAGNeR, G. (1989). Measurement of 13C spin-spin relaxation times by two-dimensional heteronuclear 1H·13C correlation spectroscopy. Journal of Magnetic Resonance (1969) 82, 659-661.

Niu, X., Bruschweiler-Li, L., Davulcu, O., SKalicky, J. J., Brüschweiler, R. \& Chapman, M. S. (2011). Arginine kinase: joint crystallographic and NMR RDC analyses link substrate-associated motions to intrinsic flexibility. Journal of Molecular Biology 405, 479-496.

Northrup, S. H., Boles, J. O. \& Reynolds, J. C. L. (1988). Brownian dynamics of cytochrome-C and cytochrome-C peroxidase association. Science 241, 67-70.

Оh, B. H., Pandit, J., Kang, C. H., Nikaido, K., Gokcen, S., Ames, G. F. L. \& Kim, S. H. (1993). 3-dimensional structures of the Periplasmic lysine arginine ornithine-binding protein with and without a ligand. Journal of Biological Chemistry 268, 11348-11355.

Olsson, U. \& Wolf-Watz, M. (2010). Overlap between folding and functional energy landscapes for adenylate kinase conformational change. Nature Communications 1, Article number 111.

Onuchic, J. N. \& Wolynes, P. G. (1993). Energy landscapes, glass transitions, and chemical-reaction dynamics in biomolecular or solvent environment. Journal of Chemical Physics 98, 2218-2224.

Ottiger, M. \& BaX, A. (1998). Determination of relative N-H-N N-C', C-alpha-C', and C(alpha)-H-alpha effective bond lengths in a protein by NMR in a dilute liquid crystalline phase. Journal of the American Chemical Society 120, 12334-12341.

Ottiger, M., Delaglio, F. \& BaX, A. (1998). Measurement of J and dipolar couplings from simplified two-dimensional NMR spectra. Journal of Magnetic Resonance 131, 373-378.

PaCI, E. \& Karplus, M. (1999). Forced unfolding of fibronectin type 3 modules: an analysis by biased molecular dynamics simulations1. Journal of Molecular Biology 288, 441-459.

Palmer, A. G. (2004). NMR characterization of the dynamics of biomacromolecules. Chemical Reviews 104, 3623-3640.

Palmer, A. G. III (2015). Enzyme dynamics from NMR spectroscopy. Accounts of Chemical Research 48, 457-465.

Palmer, A. G. III, KroenKe, C. D. \& Loria, J. P. (2001). Nuclear magnetic resonance methods for quantifying microsecond-to-millisecond motions in biological macromolecules. Energetics of Biological Macromolecules, Pt E 339, 204-238.

PAlmer, A. G. \& MAssi, F. (2006). Characterization of the dynamics of biomacromolecules using rotating-frame spin relaxation NMR spectroscopy. Chemical Reviews 106, 1700-1719.

Palmer, A. G., RanCE, M. \& Wright, P. E. (1991). Intramolecular motions of a zinc finger DNA-binding domain from Xfin characterized by proton-detected natural abundance carbon-13 heteronuclear NMR spectroscopy. Journal of the American Chemical Society 113, 4371-4380.

Park, S. H., Das, B. B., Casagrande, F., Tian, Y., Nothnagel, H. J., Chu, M., Kiefer, H., Maier, K., De Angelis, A. A., Marassi, F. M. \& Opella, S. J. (2012). Structure of the chemokine receptor CXCR1 in phospholipid bilayers. Nature 491, 779-783.

Park, S. J., Borin, B. N., Martinez-Yamout, M. A. \& Dyson, H. J. (2011). The client protein p53 adopts a molten globule-like state in the presence of Hsp90. Nature Structural \& Molecular Biology 18, 537-541.

Parker, D., Ferreri, K., Nakajima, T., Morte, V. J. L., Evans, R., Koerber, S. C., Hoeger, C. \& Montminy, M. R. (1996). Phosphorylation of CREB at Ser-133 induces complex formation with CREB-binding protein via a direct mechanism. Molecular and Cellular Biology 16, 694-703.

Parkinson, J. S. \& Koford, E. C. (1992). Communication modules in bacterial signaling proteins. Annual Review of Genetics 26, 71-112.

Passner, J.M., Schultz, S.C. \& Steitz, T.A. (2000). Modeling the cAMP-induced allosteric transition using the crystal structure of CAP-cAMP at 2.1 angstrom resolution. Journal of Molecular Biology 304, 847-859.

Pelletier, H. \& Kraut, J. (1992). Crystal-structure of a complex between electron-transfer partners, cytochrome-C peroxidase and cytochrome-C. Science 258, 1748-1755.

Pervushin, K., Riek, R., Wider, G. \& WUthrich, K. (1997). Attenuated T2 relaxation by mutual cancellation of dipole-dipole coupling and chemical shift anisotropy indicates an avenue to NMR structures of very large biological macromolecules in solution. Proceedings of the National Academy of Sciences of the United States of America 94, 12366-12371.

Peti, W., Meiler, J., Bruschweiler, R. \& Griesinger, C. (2002). Model-free analysis of protein backbone motion from residual dipolar couplings. Journal of the American Chemical Society 124, 5822-5833.

Phillips, A. H., Schoeffler, A. J., Matsui, T., Weiss, T. M., Blankenship, J. W., Zobel, K., Giannetti, A. M., Dueber, E. C. \& Fairbrother, W. J. (2014). Internal motions prime cIAP1 for rapid activation. Nature Structural \& Molecular Biology 21, 1068-1074.

Pontiggia, F., Pachov, D. V., Clarkson, M. W., Villali, J., Hagan, M. F., Pande, V. S. \& Kern, D. (2015). Free energy landscape of activation in a signalling protein at atomic resolution. Nature Communications 6, 7284.

Popovych, N., Sun, S. J., Ebright, R. H. \& Kalodimos, C. G. (2006). Dynamically driven protein allostery. Nature Structural \& Molecular Biology 13, 831-838.

Popovych, N., Tzeng, S. R., Tonelli, M., Ebright, R. H. \& Kalodimos, C. G. (2009). Structural basis for cAMP-mediated allosteric control of the catabolite activator protein. Proceedings of the National Academy of Sciences of the United States of America 106, 6927-6932.

Purcell, E. M., Torrey, H. C. \& Pound, R. V. (1946). Resonance absorption by nuclear magnetic moments in a solid. Physical Review 69, 37-38.

Qin, F., Jiang, Y. B., Chen, Y., Wu, M. Y., Yan, G. W., Ye, W. J., Li, Y.X., Zhang, J. A. \& Chen, H. F. (2011). Conformational selection or induced fit for Brinker and DNA recognition. Physical Chemistry Chemical Physics 13, 1407-1412.

Radhakrishnan, I., Perez-Alvarado, G. C., Parker, D., Dyson, H. J., Montminy, M. R. \& Wright, P. E. (1997). Solution structure of the KIX domain of CBP bound to the transactivation domain of CREB: a model for activator:coactivator interactions. Cell 91, 741-752. 
Radoicic, J., Lu, G. J. \& OpelLA, S. J. (2014). NMR structures of membrane proteins in phospholipid bilayers. Quarterly Reviews of Biophysics 47, 249-283.

Radzicka, A. \& Wolfenden, R. (1995). A proficient enzyme. Science 267, 90-93.

Ramachandran, P. L., Lovett, J. E., Carl, P. J., Cammarata, M., Lee, J. H., Jung, Y. O., Ihee, H., Timmel, C. R. \& Van Thor, J. J. (2011). The short-lived signaling state of the photoactive yellow protein photoreceptor revealed by combined structural probes. Journal of the American Chemical Society 133, 9395-9404.

ReDFieLd, A. G. (1957). On the theory of relaxation processes. IBM Journal of Research and Development 1, 19-31.

Redfield, A. G. (1965). The theory of relaxation processes. In Advances in Magnetic and Optical Resonance, vol. 1 (ed. S. W. JoHN), pp. 1-32. New York, USA: Academic Press.

ReNNELla, E., SOlyom, Z. \& BRUTSCheR, B. (2014). Measuring hydrogen exchange in proteins by selective water saturation in 1H-15N SOFAST/ BEST-type experiments: advantages and limitations. Journal of Biomolecular NMR 60, 99-107.

Rhoads, D. G. \& Lowenstein, J. M. (1968). Initial velocity and equilibrium kinetics of myokinase. The Journal of Biological Chemistry 243, 3963-3972.

Richter, K. \& Buchner, J. (2001). Hsp90: chaperoning signal transduction. Journal of Cellular Physiology 188, 281-290.

Riek, R., Fiaux, J., Bertelsen, E. B., Horwich, A. L. \& WÜthrich, K. (2002). Solution NMR techniques for large molecular and supramolecular structures. Journal of the American Chemical Society 124, 12144-12153.

Robic, S., Guzman-Casado, M., Sanchez-Ruiz, J. M. \& Marqusee, S. (2003). Role of residual structure in the unfolded state of a thermophilic protein. Proceedings of the National Academy of Sciences of the United States of America 100, 11345-11349.

Robustelli, P., StAfFord, K. A. \& PAlmer, A. G. (2012). Interpreting protein structural dynamics from NMR chemical shifts. Journal of the American Chemical Society 134, 6365-6374.

RocHE, J., LouIs, J. M. \& BAX, A. (2015). Conformation of inhibitor-free HIV-1 protease derived from NMR spectroscopy in a weakly oriented solution. Chembiochem 16, 214-218.

Roder, H., Elove, G. A. \& Englander, S. W. (1988). Structural characterization of folding intermediates in cytochrome c by H-exchange labelling and proton NMR. Nature 335, 700-704.

Rodgers, L., Gamez, A., Riek, R. \& GHosh, P. (2008). The type III secretion chaperone SycE promotes a localized disorder-to-order transition in the natively unfolded effector YopE. The Journal of Biological Chemistry 283, 20857-20863.

Rogne, P., Sparrman, T., Anugwom, I., Mikkola, J. P. \& Wolf-Watz, M. (2015). Real-time 31P NMR investigation on the catalytic behavior of the enzyme Adenylate kinase in the matrix of a switchable ionic liquid. ChemSusChem 8, 3764-3768.

Ruan, K. \& Tolman, J. R. (2005). Composite alignment media for the measurement of independent sets of NMR residual dipolar couplings. Journal of the American Chemical Society 127, 15032-15033.

Rudiger, S., Freund, S. M. V., Veprintsev, D. B. \& Fersht, A. R. (2002). CRinePT-TrOSY NMR reveals p53 core domain bound in an unfolded form to the chaperone Hsp90. Proceedings of the National Academy of Sciences of the United States of America 99, 11085-11090.

Rundqvist, L., Ådén, J., Sparrman, T., Wallgren, M., Olsson, U. \& Wolf-Watz, M. (2009). Noncooperative folding of subdomains in Adenylate Kinase. Biochemistry 48, 1911-1927.

Russo, N. \& Shapiro, R. (1999). Potent inhibition of mammalian ribonucleases by 3', 5'-pyrophosphate-linked nucleotides. The Journal of Biological Chemistry 274, 14902-14908.

Saio, T., Guan, X., Rossi, P., Economou, A. \& Kalodimos, C. G. (2014). Structural basis for protein antiaggregation activity of the trigger factor chaperone. Science 344, 1250494.

Salvatella, X., Richter, B. \& Vendruscolo, M. (2008). Influence of the fluctuations of the alignment tensor on the analysis of the structure and dynamics of proteins using residual dipolar couplings. Journal of Biomolecular NMR 40, 71-81.

Sanchez-Medina, C., Sekhar, A., Vallurupalli, P., Cerminara, M., Munoz, V. \& Kay, L. E. (2014). Probing the free energy landscape of the fast-folding gpW protein by relaxation dispersion NMR. Journal of the American Chemical Society 136, 7444-7451.

SAPIEnZA, P. J. \& LeE, A.L. (2010). Using NMR to study fast dynamics in proteins: methods and applications. Current Opinion in Pharmacology 10, 723-730.

Sass, H. J., Musco, G., Stahl, S. J., Wingfield, P. T. \& GrZesiek, S. (2000). Solution NMR of proteins within polyacrylamide gels: diffusional properties and residual alignment by mechanical stress or embedding of oriented purple membranes. Journal of Biomolecular NMR 18, 303-309.

Sauer, F. G., Futterer, K., Pinkner, J. S., Dodson, K. W., Hultgren, S. J. \& Waksman, G. (1999). Structural basis of chaperone function and pilus biogenesis. Science 285, 1058-1061.

SAWAya, M. R. \& Kraut, J. (1997). Loop and subdomain movements in the mechanism of Escherichia coli dihydrofolate reductase: crystallographic evidence. Biochemistry 36, 586-603.

SCHANDA, P. \& BRUTSCheR, B. (2005). Very fast two-dimensional NMR spectroscopy for real-time investigation of dynamic events in proteins on the time scale of seconds. Journal of the American Chemical Society 127, 8014-8015.

SCHANDA, P., KuPCE, E. \& BRUTSCHER, B. (2005). SOFAST-HMQC experiments for recording two-dimensional heteronuclear correlation spectra of proteins within a few seconds. Journal of Biomolecular NMR 33, 199-211.

SChilder, J., Lohr, F., SCHWALBE, H. \& UbBinK, M. (2014). The cytochrome c peroxidase and cytochrome c encounter complex: the other side of the story. FEBS Letters 588, 1873-1878.

Schneider, R., Maurin, D., Communie, G., Kragelj, J., Hansen, D. F., Ruigrok, R. W. H., Jensen, M. R. \& Blackledge, M. (2015). Visualizing the molecular recognition trajectory of an intrinsically disordered protein using multinuclear relaxation dispersion NMR. Journal of the American Chemical Society 137, 1220-1229. 
Schnell, J. R., Dyson, H. J. \& Wright, P. E. (2004). Structure, dynamics, and catalytic function of dihydrofolate reductase. Annual Review of Biophysics and Biomolecular Structure 33, 119-140.

Schrank, T. P., Bolen, D. W. \& Hilser, V. J. (2009). Rational modulation of conformational fluctuations in adenylate kinase reveals a local unfolding mechanism for allostery and functional adaptation in proteins. Proceedings of the National Academy of Sciences of the United States of America 106, 16984-16989.

SCHreIBER, G. (2002). Kinetic studies of protein-protein interactions. Current Opinion in Structural Biology 12, $41-47$.

Schreiber, G. \& Fersht, A. R. (1996). Rapid, electrostatically assisted association of proteins. Nature Structural Biology 3, $427-431$.

Schultz, S. C., Shields, G. C. \& Steitz, T. A. (1991). Crystal-structure of a cap-DNA complex - the DNA is bent by 90 -degrees. Science 253, $1001-1007$

SCHWieters, C. D. \& CloRe, G. M. (2001). Internal coordinates for molecular dynamics and minimization in structure determination and refinement. Journal of Magnetic Resonance 152, 288-302.

Schwieters, C. D., Kuszewski, J. J. \& Clore, G. M. (2006). Using Xplor-NIH for NMR molecular structure determination. Progress in Nuclear Magnetic Resonance Spectroscopy 48, 47-62.

Seewald, M. J., Pichumani, K., Stowell, C., Tibbals, B. V., Regan, L. \& Stone, M. J. (2000). The role of backbone conformational heat capacity in protein stability: temperature dependent dynamics of the B1 domain of Streptococcal protein G. Protein Science 9, 1177-1193.

Selvaratnam, R., Vanschouwen, B., Fogolari, F., Mazhab-Jafari, M. T., Das, R. \& Melacini, G. (2012). The projection analysis of NMR chemical shifts reveals extended EPAC autoinhibition determinants. Biophysical Journal 102, 630-639.

Serber, Z., Corsini, L., Durst, F. \& Dotsch, V. (2005). In-cell NMR spectroscopy. In Nuclear Magnetic Resonance of Biological Macromolecules, Part C, vol. 394 (ed. T. L. JAMES), pp. 17-41. San Diego, USA: Elsevier Academic Press Inc.

Serber, Z., Selenko, P., Hansel, R., Reckel, S., Lohr, F., Ferrell, J. E., Wagner, G. \& Dotsch, V. (2007). Investigating macromolecules inside cultured and injected cells by in-cell NMR spectroscopy. Nature Protocols 1, 2701-2709.

Sgourakis, N. G., Lange, O. F., Dimaio, F., André, I., Fitzkee, N. C., Rossi, P., Montelione, G. T., BaX, A. \& Baker, D. (2011). Determination of the structures of symmetric protein oligomers from NMR chemical shifts and residual dipolar couplings. Journal of the American Chemical Society 133, 6288-6298.

Shan, Y., Arkhipov, A., Kim, E. T., Pan, A. C. \& Shaw, D. E. (2013). Transitions to catalytically inactive conformations in EGFR kinase. Proceedings of the National Academy of Sciences of the United States of America 110, 7270-7275.

Shapiro, Y. E., Kahana, E. \& Meirovitch, E. (2009). Domain mobility in proteins from NMR/SRLS. Journal of Physical Chemistry B 113, 12050-12060.

Shapiro, Y. E. \& Meirovitch, E. (2006). Activation energy of catalysis-related domain motion in E. coli adenylate kinase. Journal of Physical Chemistry B Condens Matter Mater Surf Interfaces Biophys 110, 11519-11524.

Shapiro, Y. E., Sinev, M. A., Sineva, E. V., Tugarinov, V. \& Meirovitch, E. (2000). Backbone dynamics of Escherichia coli adenylate kinase at the extreme stages of the catalytic cycle studied by (15)N NMR relaxation. Biochemistry 39, 6634-6644.

Sharff, A. J., Rodseth, L. E., Spurlino, J. C. \& Quiocho, F. A. (1992). Crystallographic evidence of a large ligand-induced hinge-twist motion between the 2 domains of the maltodextrin binding-protein involved in active-transport and chemotaxis. Biochemistry 31, 10657-10663.

SHEN, Y. \& BAX, A. (2015). Homology modeling of larger proteins guided by chemical shifts. Nature Methods 12, 747-750.

Shen, Y., Bryan, P. N., He, Y., Orban, J., BaKer, D. \& BaX, A. (2010). De novo structure generation using chemical shifts for proteins with high-sequence identity but different folds. Protein Science 19, 349-356.

Sher, I., Chang, S. C., Li, Y., Chhabra, S., Palmer, A. G., Norton, R. S. \& Chill, J. H. (2014). Conformational flexibility in the binding surface of the potassium channel blocker ShK. Chembiochem 15, 2402-2410.

Sibille, N. \& Bernado, P. (2012). Structural characterization of intrinsically disordered proteins by the combined use of NMR and SAXS Biochemical Society Transactions 40, 955-962.

Silvestre-Ryan, J., Bertoncini, C. W., Fenwick, R. B., Esteban-Martin, S. \& Salvatella, X. (2013). Average conformations determined from PRE data provide high-resolution maps of transient tertiary interactions in disordered proteins. Biophysical Journal 104, $1740-1751$.

Skinner, J. J., Lim, W. K., Bédard, S., Black, B. E. \& Englander, S. W. (2012a). Protein dynamics viewed by hydrogen exchange. Protein Science 21, 996-1005.

Skinner, J. J., Lim, W. K., Bédard, S., Black, B. E. \& Englander, S. W. (2012b). Protein hydrogen exchange: testing current models. Protein Science 21, 987-995.

SKrynnikov, N. R., Dahlquist, F. W. \& Kay, L. E. (2002). Reconstructing NMR spectra of "invisible" excited protein states using HSQC and HMQC experiments. Journal of the American Chemical Society 124, 12352-12360.

Skrynnikov, N. R., Mulder, F. A. A., Hon, B., Dahlquist, F. W. \& Kay, L. E. (2001). Probing slow time scale dynamics at methyl-containing side chains in proteins by relaxation dispersion NMR measurements: application to methionine residues in a cavity mutant of T4 lysozyme. Journal of the American Chemical Society 123, 4556-4566.

Smith, A. E., Sarkar, M., Young, G. B. \& Pielak, G. J. (2013). Amide proton exchange of a dynamic loop in cell extracts. Protein Science 22, 1313-1319.

Solomon, I. (1955). Relaxation processes in a system of 2 SPINS. Physical Review 99, 559-565.

SPOLAR, R. \& ReCORD, M. (1994). Coupling of local folding to site-specific binding of proteins to DNA. Science 263, 777-784.

Sprangers, R., Gribun, A., Hwang, P. M., Houry, W. A. \& Kay, L. E. (2005). Quantitative NMR spectroscopy of supramolecular complexes: dynamic side pores in ClpP are important for product release. Proceedings of the National Academy of Sciences of the United States of America 102, 16678-16683. 
Sprangers, R. \& Kay, L. E. (2007). Quantitative dynamics and binding studies of the 20S proteasome by NMR. Nature 445, 618-622.

Sprangers, R., Velyvis, A. \& Kay, L.E. (2007). Solution NMR of supramolecular complexes: providing new insights into function. Nature Methods 4, 697-703.

Spurlino, J. C., Lu, G. Y. \& Quiocho, F. A. (1991). The 2-3-Å resolution structure of the maltose-binding or maltodextrin-binding protein, a primary receptor of bacterial active-transport and chemotaxis. Journal of Biological Chemistry 266, 5202-5219.

Stafford, K. A., Robustelli, P. \& Palmer, A.G. III (2013). Thermal adaptation of conformational dynamics in ribonuclease H. PLoS Computational Biology 9, e1003218.

Stan, G., Brooks, B. R., Lorimer, G. H. \& Thirumalai, D. (2006). Residues in substrate proteins that interact with GroEL in the capture process are buried in the native state. Proceedings of the National Academy of Sciences of the United States of America 103, 4433-4438.

Stewart, M. D. \& Igumenova, T. I. (2012). Reactive cysteine in the structural Zn2+ site of the C1B domain from PKC $\alpha$. Biochemistry 51, $7263-7277$.

Stevens, S. Y., Sanker, S., Kent, C. \& Zuiderweg, E. R. (2001). Deliniation of the allosteric mechanism of a cytidyltransferase exhibiting negative cooperativity. Nature Structural Biology 8, 947-952.

Stone, M. J. (2001). NMR relaxation studies of the role of conformational entropy in protein stability and ligand binding. Accounts of Chemical Research 34, 379-388.

Sugase, K., Dyson, H. J. \& Wright, P. E. (2007). Mechanism of coupled folding and binding of an intrinsically disordered protein. Nature 447, 1021-U1011.

Sullivan, S. M. \& HolYoAK, T. (2008). Enzymes with lid-gated active sites must operate by an induced fit mechanism instead of conformational selection. Proceedings of the National Academy of Sciences of the United States of America 105, 13829-13834.

Szyperski, T., Luginbühl, P., Otting, G., Güntert, P. \& Wüthrich, K. (1993). Protein dynamics studied by rotating frame 15N spin relaxation times. Journal of Biomolecular NMR 3, 151-164.

TaKetomi, H., Ueda, Y. \& Go, N. (1975). Studies on protein folding, unfolding and fluctuations by computer-simulation .1. Effect of specific amino-acid sequence represented by specific inter-unit interactions. International Journal of Peptide and Protein Research 7, 445-459.

TANG, C., Iwahara, J. \& Clore, G. M. (2006). Visualization of transient encounter complexes in protein-protein association. Nature 444, $383-386$.

TANG, C., SchwieTers, C. D. \& Clore, G. M. (2007). Open-to-closed transition in apo maltose-binding protein observed by paramagnetic NMR. Nature 449, 1078-U1012.

Thongwichian, R., Kosten, J., Benary, U., Rose, H. M., Stuiver, M., Theillet, F.-X., Dose, A., Koch, B., Yokoyama, H., Schwarzer, D., Wolf, J. \& SeLENKO, P. (2015). A multiplexed NMR-reporter approach to measure cellular kinase and phosphatase activities in real-time. Journal of the American Chemical Society 137, 6468-6471.

TJandra, N. \& BaX, A. (1997). Direct measurement of distances and angles in biomolecules by NMR in a dilute liquid crystalline medium. Science 278, 1111-1114.

TJandra, N., GrZesiek, S. \& BaX, A. (1996). Magnetic field dependence of nitrogen-proton J Splittings in 15N-Enriched human ubiquitin resulting from relaxation interference and residual dipolar coupling. Journal of the American Chemical Society 118, 6264-6272.

Tjandra, N., Omichinski, J. G., Gronenborn, A. M., Clore, G. M. \& BaX, A. (1997). Use of dipolar 1H-15N and 1H-13C couplings in the structure determination of magnetically oriented macromolecules in solution. Nature Structural Biology 4, 732-738.

Tollinger, M., Skrynnikov, N. R., Mulder, F. A., Forman-Kay, J. D. \& Kay, L. E. (2001). Slow dynamics in folded and unfolded states of an SH3 domain. Journal of the American Chemical Society 123, 11341-11352.

Tolman, J. R. (2002). A novel approach to the retrieval of structural and dynamic information from residual dipolar couplings using several oriented media in biomolecular NMR spectroscopy. Journal of the American Chemical Society 124, 12020-12030.

Tolman, J. R., Al-Hashimi, H. M., Kay, L. E. \& Prestegard, J. H. (2001). Structural and dynamic analysis of residual dipolar coupling data for proteins. Journal of the American Chemical Society 123, 1416-1424.

Tolman, J. R., Flanagan, J. M., Kennedy, M. A. \& Prestegard, J. H. (1995). Nuclear magnetic dipole interactions in field-oriented proteins information for structure determination in solution. Proceedings of the National Academy of Sciences of the United States of America 92, 9279-9283.

Tolman, J. R., Flanagan, J. M., Kennedy, M. A. \& Prestegard, J. H. (1997). NMR evidence for slow collective motions in cyanometmyoglobin. Nature Structural Biology 4, 292-297.

Trbovic, N., Cho, J.-H., Abel, R., Friesner, R. A., Rance, M. \& Palmer, A. G. (2009). Protein side-chain dynamics and residual conformational entropy. Journal of the American Chemical Society 131, 615-622.

Tripathi, S. \& Portman, J. J. (2009). Inherent flexibility determines the transition mechanisms of the EF-hands of calmodulin. Proceedings of the National Academy of Sciences of the United States of America 106, 2104-2109.

Trott, O. \& Palmer, A. G. III (2002). R1 $\rho$ relaxation outside of the fast-exchange limit. Journal of Magnetic Resonance 154, 157-160.

Tugarinov, V., Hwang, P. M. \& KaY, L.E. (2004). Nuclear magnetic resonance spectroscopy of high-molecular-weight proteins. Annual Review of Biochemistry 73, 107-146.

Tugarinov, V., Hwang, P. M., Ollerenshaw, J. E. \& Kay, L. E. (2003). Cross-correlated relaxation enhanced 1H-13C NMR spectroscopy of methyl groups in very high molecular weight proteins and protein complexes. Journal of the American Chemical Society 125, 10420-10428.

Tycko, R., Blanco, F. J. \& IshiI, Y. (2000). Alignment of biopolymers in strained gels: a new way to create detectable dipole-dipole couplings in high-resolution biomolecular NMR. Journal of the American Chemical Society 122, 9340-9341.

Tyler, R. C., Wieting, J. C., Peterson, F. C. \& Volkman, B. F. (2012). Electrostatic optimization of the conformational energy landscape in a metamorphic protein. Biochemistry 51, 9067-9075. 
Tzeng, S.-R. \& Kalodimos, C. G. (2009). Dynamic activation of an allosteric regulatory protein. Nature 462, 368-372.

Tzeng, S.-R. \& Kalodimos, C. G. (2013). Allosteric inhibition through suppression of transient conformational states. Nature Chemical Biology 9, 462-465.

UdgaonkaR, J. B. \& BALDWIn, R. L. (1990). Early folding intermediate of ribonuclease A. Proceedings of the National Academy of Sciences of the United States of America 87, 8197-8201.

Uesugi, M., Nyanguile, O., Lu, H., Levine, A. J. \& Verdine, G. L. (1997). Induced alpha helix in the VP16 activation domain upon binding to a human TAF. Science 277, 1310-1313.

Ullrich, S. J. \& Glaubitz, C. (2013). Perspectives in enzymology of membrane proteins by solid-state NMR. Accounts of Chemical Research 46, 2164-2171.

Ulmer, T. S., BaX, A., Cole, N. B. \& Nussbaum, R. L. (2005). Structure and dynamics of micelle-bound human alpha-synuclein. Journal of Biological Chemistry 280, 9595-9603.

Ulmer, T. S., Ramirez, B. E., Delaglio, F. \& Bax, A. (2003). Evaluation of backbone proton positions and dynamics in a small protein by liquid crystal NMR spectroscopy. Journal of the American Chemical Society 125, 9179-9191.

Wahlberg, E., Lendel, C., Helgstrand, M., Allard, P., Dincbas-Renqvist, V., Hedqvist, A., Berglund, H., Nygren, P.-Å. \& Härd, T. (2003). An affibody in complex with a target protein: structure and coupled folding. Proceedings of the National Academy of Sciences of the United States of America 100, 3185-3190.

Vallurupali, P., Bouvignies, G. \& Kay, L. E. (2011). Increasing the exchange time-scale that can be probed by CPMG relaxation dispersion NMR. Journal of Physical Chemistry B 115, 14891-14900.

Vallurupalli, P., Bouvignies, G. \& Kay, L. E. (2012). Studying "invisible" excited protein states in slow exchange with a major state conformation. Journal of the American Chemical Society 134, 8148-8161.

Vallurupalli, P., Hansen, D. F., Stollar, E., Meirovitch, E. \& Kay, L. E. (2007). Measurement of bond vector orientations in invisible excited states of proteins. Proceedings of the National Academy of Sciences of the United States of America 104, 18473-18477.

Vallurupalli, P. \& Kay, L. E. (2013). Probing slow chemical exchange at carbonyl sites in proteins by chemical exchange saturation transfer NMR spectroscopy. Angewandte Chemie-International Edition 52, 4156-4159.

VAmmi, V., Lin, T.-L. \& Song, G. (2014). Enhancing the quality of protein conformation ensembles with relative populations. Journal of Biomolecular NMR 58, 209-225.

Vanatta, D. K., Shukla, D., Lawrenz, M. \& Pande, V. S. (2015). A network of molecular switches controls the activation of the two-component response regulator NtrC. Nature Communications 6, Article number 7283.

Veglia, G. \& Cembran, A. (2013). Role of conformational entropy in the activity and regulation of the catalytic subunit of protein kinase A. Febs Journal 280, 5608-5615.

VIJAY-KumaR, S., BugG, C. E. \& СooK, W. J. (1987). Structure of ubiquitin refined at 1.8Åresolution. Journal of Molecular Biology 194, 531-544.

Vogt, A. D. \& Di CerA, E. (2012). Conformational selection or induced fit? A critical appraisal of the kinetic mechanism. Biochemistry 51, 5894-5902.

Volkman, B. F., Lipson, D., Wemmer, D. E. \& Kern, D. (2001). Two-state allosteric behavior in a single-domain signaling protein. Science 291, 2429-2433.

Volkman, B. F., Nohaile, M. J., Amy, N. K., Kustu, S. \& Wemmer, D. E. (1995). Three-dimensional solution structure of the N-terminal receiver domain of NTRC. Biochemistry 34, 1413-1424.

Volkov, A. N., Worrall, J. A. R., Holtzmann, E. \& Ubbink, M. (2006). Solution structure and dynamics of the complex between cytochrome c and cytochrome c peroxidase determined by paramagnetic NMR. Proceedings of the National Academy of Sciences of the United States of America 103, 18945-18950.

WAND, A. J. (2001). Dynamic activation of protein function: a view emerging from NMR spectroscopy. Nature Structural Biology 8, 926-931.

WAND, A. J. (2013). The dark energy of proteins comes to light: conformational entropy and its role in protein function revealed by NMR relaxation. Current Opinion in Structural Biology 23, 75-81.

Wang, C., Bradley, P. \& Baker, D. (2007). Protein-protein docking with backbone flexibility. Journal of Molecular Biology 373, 503-519.

Wang, C., Rance, M. \& Palmer, A. G. (2003). Mapping chemical exchange in proteins with MW $>50 \mathrm{kD}$. Journal of the American Chemical Society 125, 8968-8969.

Wang, Z., Feng, H.-P., Landry, S. J., MaXwell, J. \& Gierasch, L. M. (1999). Basis of substrate binding by the Chaperonin GroEL. Biochemistry 38, 12537-12546.

Wangsness, R. K. \& Bloch, F. (1953). The dynamical theory of nuclear induction. Physical Review 89, 728-739.

Watt, E. D., Shimada, H., Kovrigin, E. L. \& Loria, J. P. (2007). The mechanism of rate-limiting motions in enzyme function. Proceedings of the National Academy of Sciences of the United States of America 104, 11981-11986.

Weise, C. F., Login, F. H., Ho, O., Grobner, G., Wolf-Watz, H. \& Wolf-Watz, M. (2014). Negatively charged lipid membranes promote a disorder-order transition in the Yersinia YscU protein. Biophysical Journal 107, 1950-1961.

Wenter, P., Fürtig, B., Hainard, A., Schwalbe, H. \& Pitsch, S. (2005). Kinetics of photoinduced RNA refolding by real-time NMR spectroscopy. Angewandte Chemie International Edition 44, 2600-2603.

Whitford, P. C. (2013). Disorder guides protein function. Proceedings of the National Academy of Sciences of the United States of America 110 , 7114-7115.

Whitford, P. C., Miyashita, O., Levy, Y. \& Onuchic, J. N. (2007). Conformational transitions of adenylate kinase: switching by cracking. Journal of Molecular Biology 366, 1661-1671. 
Whitford, P. C., ONUCHIC, J. N. \& Wolynes, P. G. (2008). Energy landscape along an enzymatic reaction trajectory: hinges or cracks? HFSP Journal 2, 61-64.

WhitTier, S. K., HengGe, A. C. \& LoRIA, J. P. (2013). Conformational motions regulate phosphoryl transfer in related protein tyrosine phosphatases. Science 341, 899-903.

Wikström, A., Berglund, H., Hambraeus, C., Van Den Berg, S. \& Härd, T. (1999). Conformational dynamics and molecular recognition: backbone dynamics of the estrogen receptor DNA-binding domain. Journal of Molecular Biology 289, 963-979.

WiLDes, D. \& MARquSEE, S. (2004). Hydrogen-exchange strategies applied to energetics of intermediate processes in protein folding. Energetics of Biological Macromolecules, Pt E 380, 328-349.

Williams, J. C. \& MсDеrmotT, A. E. (1995). Dynamics of the flexible loop of triosephosphate isomerase: the loop motion is not ligand gated. Biochemistry 34, 8309-8319.

Williamson, M. (2012). How Proteins Work. New York, USA: Garland Science, Taylor \& Francis Group LLC.

Wilson, C., Agafonov, R. V., Hoemberger, M., Kutter, S., Zorba, A., Halpin, J., Buosi, V., Otten, R., Waterman, D., Theobald, D. L. \& Kern, D. (2015). Using ancient protein kinases to unravel a modern cancer drug's mechanism. Science 347, 882-886.

Wishart, D. S., Arndt, D., Berjanskit, M., Guo, A. C., Shi, Y., Shrivastava, S., Zhou, J., Zhou, Y. \& Lin, G. (2008). PPT-DB: the protein property prediction and testing database. Nucleic Acids Research 36(Database issue), D222-229.

Wolf-Watz, M., Thai, V., Henzler-Wildman, K., Hadjipavlou, G., Eisenmesser, E. Z. \& Kern, D. (2004). Linkage between dynamics and catalysis in a thermophilic-mesophilic enzyme pair. Nature Structural \& Molecular Biology 11, 945-949.

Wright, P. E. \& Dyson, H. J. (2015). Intrinsically disordered proteins in cellular signalling and regulation. Nature reviews. Molecular Cell Biology 16, 18-29.

Wuthrich, K. (2001). The way to NMR structures of proteins. Nature Structural Biology 8, 923-925.

YANG, D. \& KAY, L. E. (1996). Contributions to conformational entropy arising from bond vector fluctuations measured from NMR-derived order parameters: application to protein folding. Journal of Molecular Biology 263, 369-382.

Yu, T. K., Shin, S. A., Kim, E. H., Kim, S., Ryu, K. S., CheOnG, H., AhN, H. C., Jon, S. \& SuH, J. Y. (2014). An unusual protein-protein interaction through coupled unfolding and binding. Angewandte Chemie-International Edition 53, 9784-9787.

Zahn, R., Perrett, S., Stenberg, G. \& Fersht, A. R. (1996). Catalysis of amide proton exchange by the molecular chaperones GroEL and SecB. Science 271, 642-645.

Zavodszky, P., Kardos, J., Svingor, A. \& Petsko, G. A. (1998). Adjustment of conformational flexibility is a key event in the thermal adaptation of proteins. Proceedings of the National Academy of Sciences of the United States of America 95, 7406-7411.

ZеEв, M. \& BALBACH, J. (2005). NMR spectroscopic characterization of millisecond protein folding by transverse relaxation dispersion measurements. Journal of the American Chemical Society 127, 13207-13212.

Zeeb, M., Jacob, M., Schindler, T. \& Balbach, J. (2003). 15N relaxation study of the cold shock protein CspB at various solvent viscosities. Journal of Biomolecular NMR 27, 221-234.

ZeEman, P. (1897). XXXII. On the influence of magnetism on the nature of the light emitted by a substance. Philosophical Magazine Series 5 43, 226-239.

Zhang, Z.-Y. (2001). Protein tyrosine phosphatases: prospects for therapeutics. Current Opinion in Chemical Biology 5, 416-423.

Zhang, Z. Y., Thieme-Sefler, A. M., Maclean, D., Mcnamara, D. J., Dobrusin, E. M., Sawyer, T. K. \& Dixon, J. E. (1993). Substrate specificity of the protein tyrosine phosphatases. Proceedings of the National Academy of Sciences of the United States of America 90, 4446-4450.

Zhou, P., Sun, L. J., Dotsch, V., Wagner, G. \& Verdine, G. L. (1998). Solution structure of the core NFATC1/DNA complex. Cell 92, 687-696.

Zidek, L., Novotny, M. V. \& Stone, M. J. (1999). Increased protein backbone conformational entropy upon hydrophobic ligand binding. Nature Structural Biology 6, 1118-1121.

Zinn-Justin, S., Berthault, P., Guenneugues, M. \& Desvaux, H. (1997). Off-resonance rf fields in heteronuclear NMR: application to the study of slow motions. Journal of Biomolecular NMR 10, 363-372.

Zor, T., De Guzman, R. N., Dyson, H. J. \& Wright, P. E. (2004). Solution structure of the KIX domain of CBP bound to the transactivation domain of c-Myb. Journal of Molecular Biology 337, 521-534.

Zweckstetter, M. \& Bax, A. (2000). Prediction of sterically induced alignment in a dilute liquid crystalline phase: aid to protein structure determination by NMR. Journal of the American Chemical Society 122, 3791-3792. 